Pendant arm functionalized benzamidinate titanium imido compounds: experimental and computational studies of their reactions with $\mathrm{CO}_{2}$

Catherine L Boyd, Eric Clot, Aldo Guiducci and Philip Mountford*

Supporting information 
Eyring plots for $\mathrm{NMe}_{2}$ methyl group exchange data in 13 and 14

(i) Eyring plot for $\mathrm{NMe}_{2}$ methyl group exchange in $\left[\mathrm{Ti}\left(\eta-\mathrm{C}_{5} \mathrm{H}_{4} \mathrm{Me}\right)\left(\mathrm{N}^{t} \mathrm{Bu}\right)\right.$

$\left.\left\{\mathrm{Me}_{3} \mathrm{SiNC}(\mathrm{Ph}) \mathrm{NCH}_{2} \mathrm{CH}_{2} \mathrm{NMe}_{2}\right\}\right](3)$

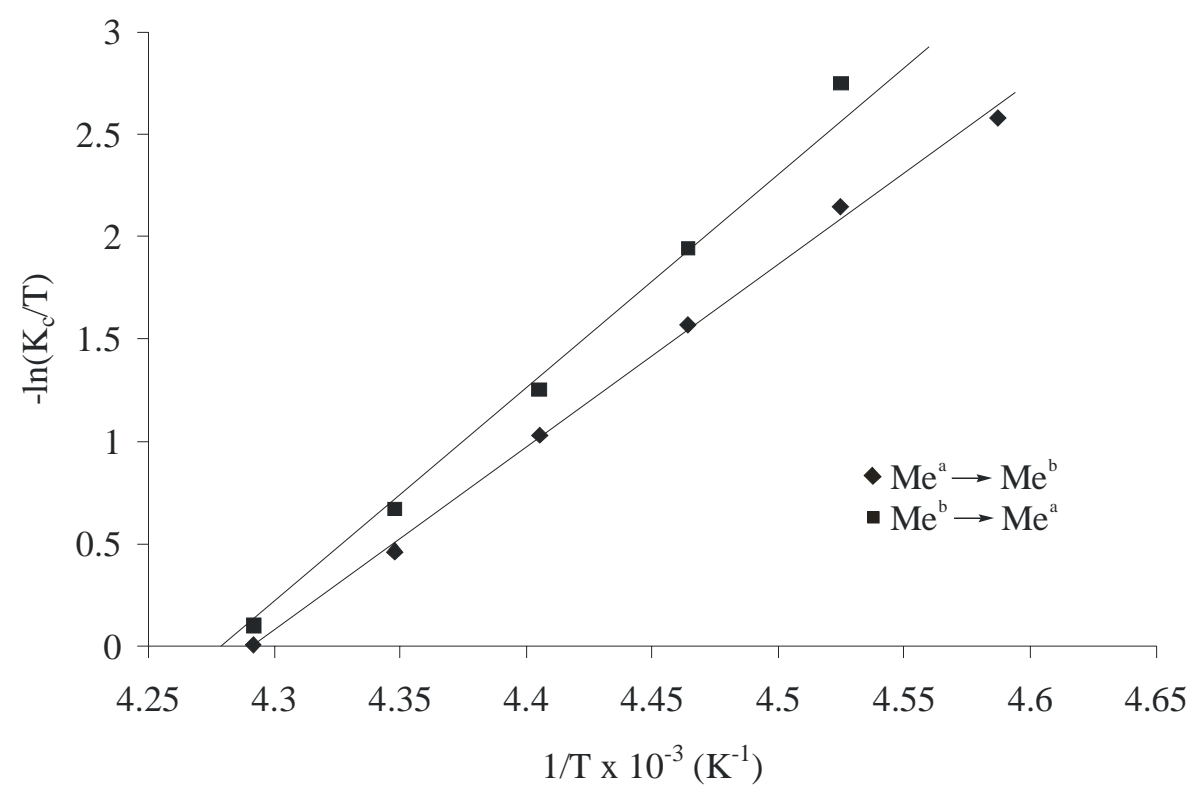

(ii) Eyring plot for $\mathrm{NMe}_{2}$ methyl group exchange in $\left[\mathrm{Ti}\left(\eta-\mathrm{C}_{5} \mathrm{H}_{4} \mathrm{Me}\right)\left(\mathrm{N}^{t} \mathrm{Bu}\right)\right.$ $\left.\left\{\mathrm{Me}_{3} \mathrm{SiNC}(\mathrm{Ph}) \mathrm{NCH}_{2} \mathrm{CH}_{2} \mathrm{CH}_{2} \mathrm{NMe}_{2}\right\}\right](4)$

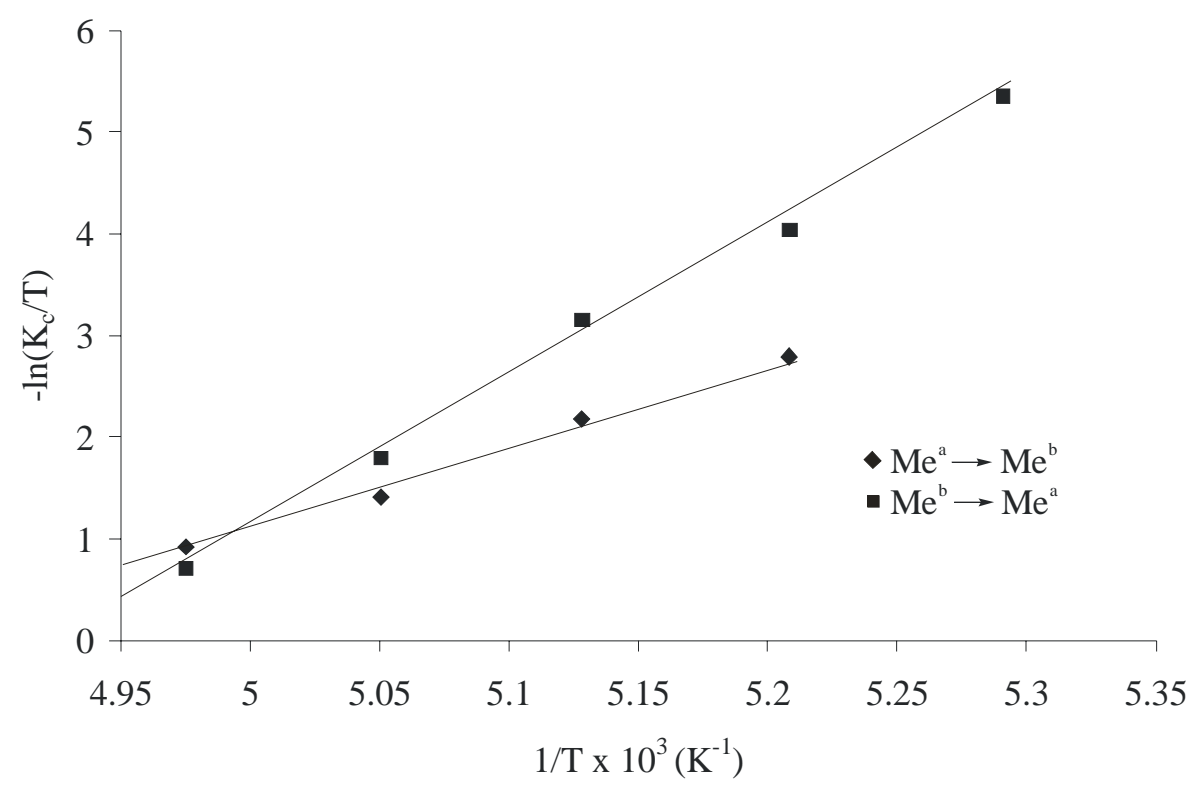


Cycloaddition reaction of $\left[\mathrm{Ti}\left(\eta-\mathrm{C}_{5} \mathrm{H}_{4} \mathrm{Me}\right)\left(\mathrm{N}^{t} \mathrm{Bu}\right)\left\{\mathrm{Me}_{3} \mathrm{SiNC}(\mathrm{Ph}) \mathrm{NCH}_{2} \mathrm{CH}_{2} \mathrm{NMe}_{2}\right\}\right]$ (3) with $\mathrm{CO}_{2}$ : kinetic studies

(i) Plot of normalised concentrations against time for the cycloaddition reaction of $\mathbf{3}$ with $\mathrm{CO}_{2}(0.25 \mathrm{~atm})$. Values are given for starting imide $\mathbf{3}$ and product carbamate $\mathbf{1 5}$

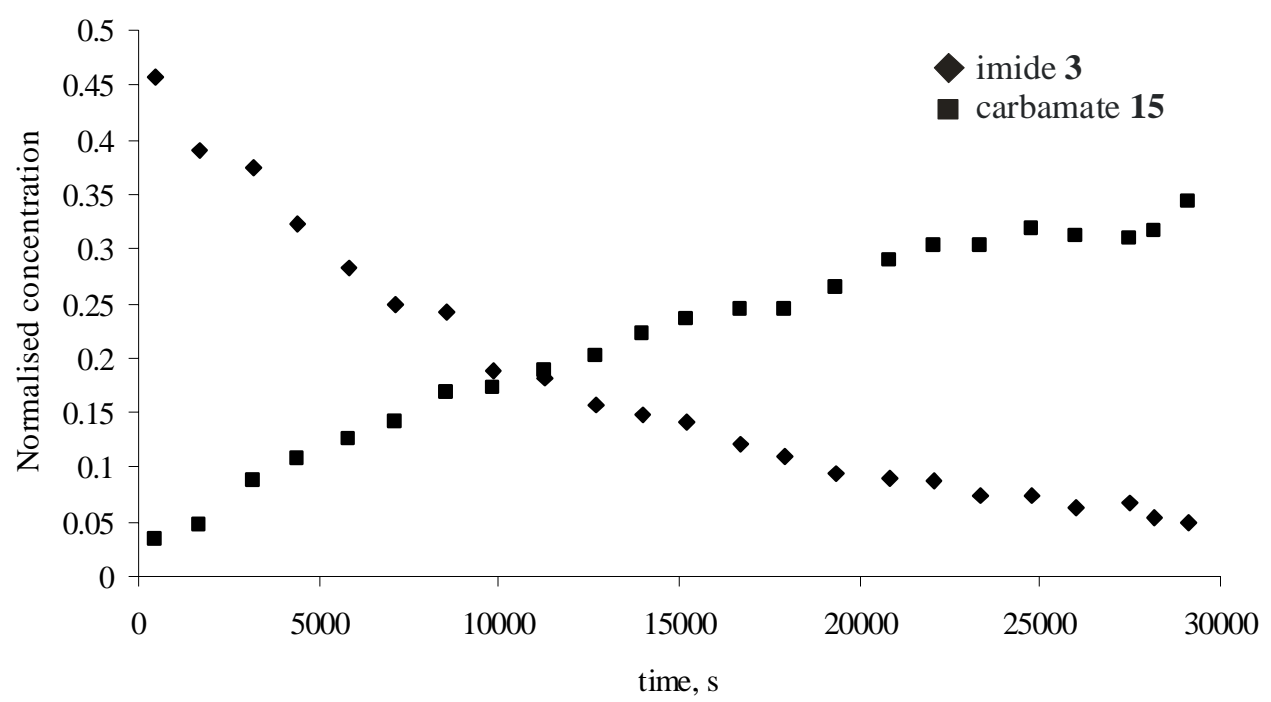

(ii) Plot of $\ln \left(\mathrm{I} / \mathrm{I}_{0}\right) v s$. time for the reaction of 3 with $\mathrm{CO}_{2}(0.25 \mathrm{~atm})$

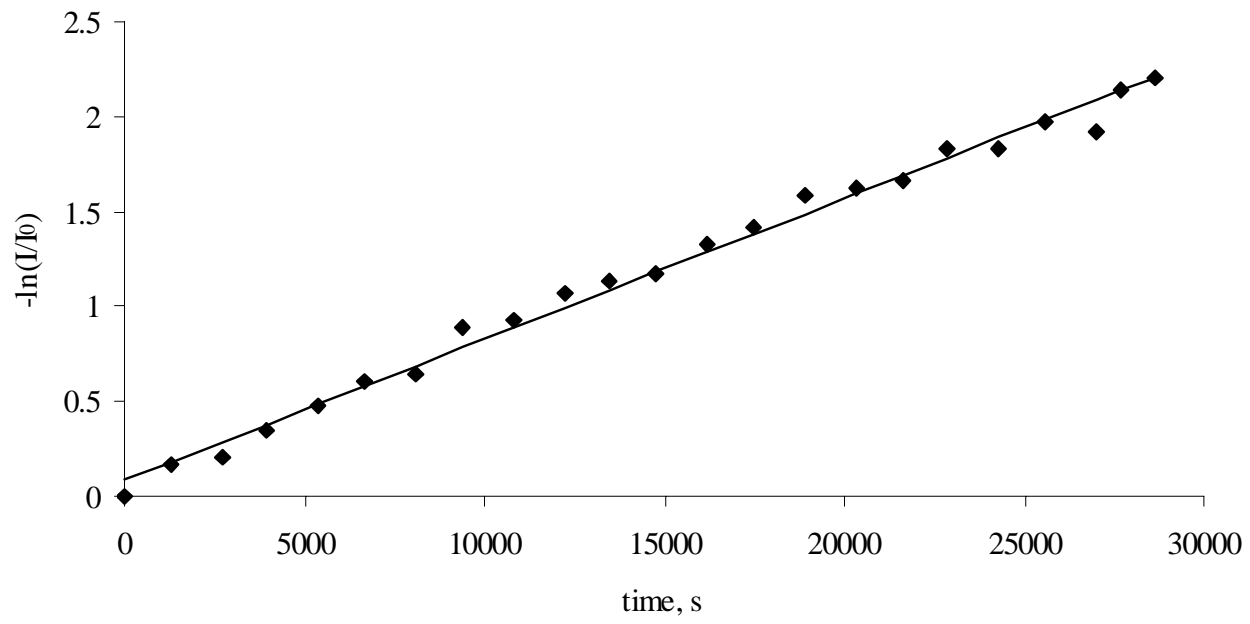


(iii) Plot of normalised concentrations against time for the cycloaddition reaction of $\mathbf{3}$ with $\mathrm{CO}_{2}(0.5 \mathrm{~atm})$. Values are given for starting imide $\mathbf{3}$ and product carbamate $\mathbf{1 5}$

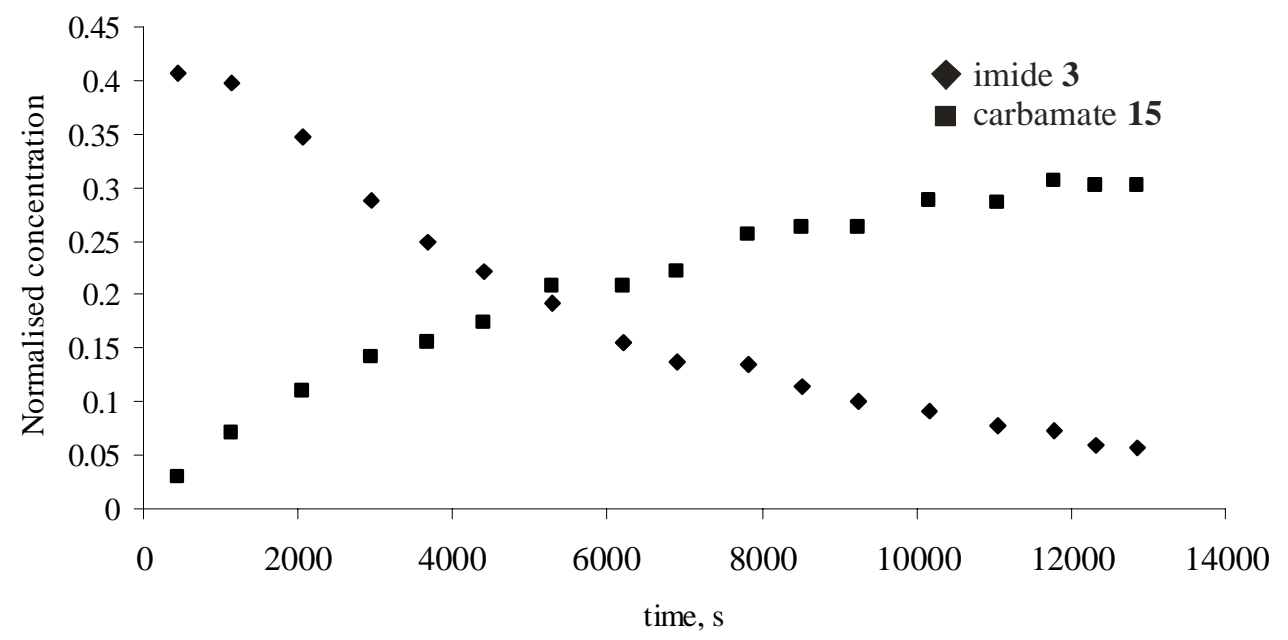

(iv) Plot of $\ln \left(\mathrm{I} / \mathrm{I}_{0}\right) v s$. time for the reaction of 3 with $\mathrm{CO}_{2}(0.5 \mathrm{~atm})$

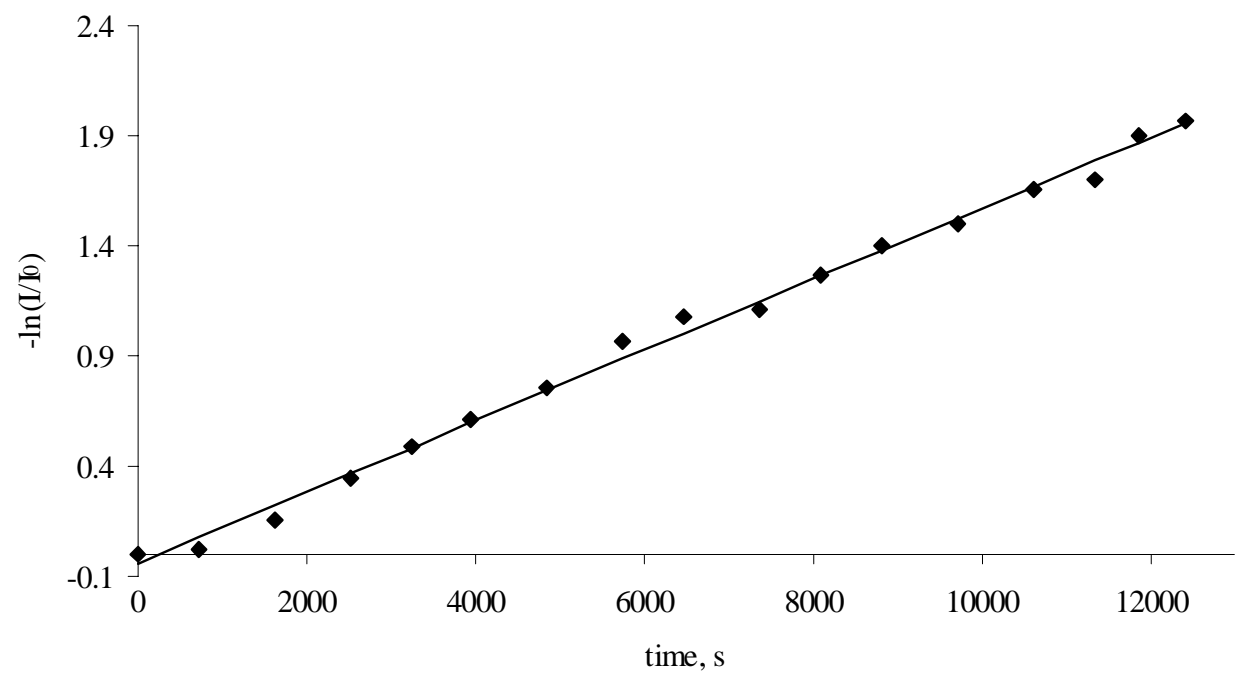


(v) Plot of normalised concentrations against time for the cycloaddition reaction of $\mathbf{3}$ with $\mathrm{CO}_{2}(0.75 \mathrm{~atm})$. Values are given for starting imide $\mathbf{3}$ and product carbamate $\mathbf{1 5}$

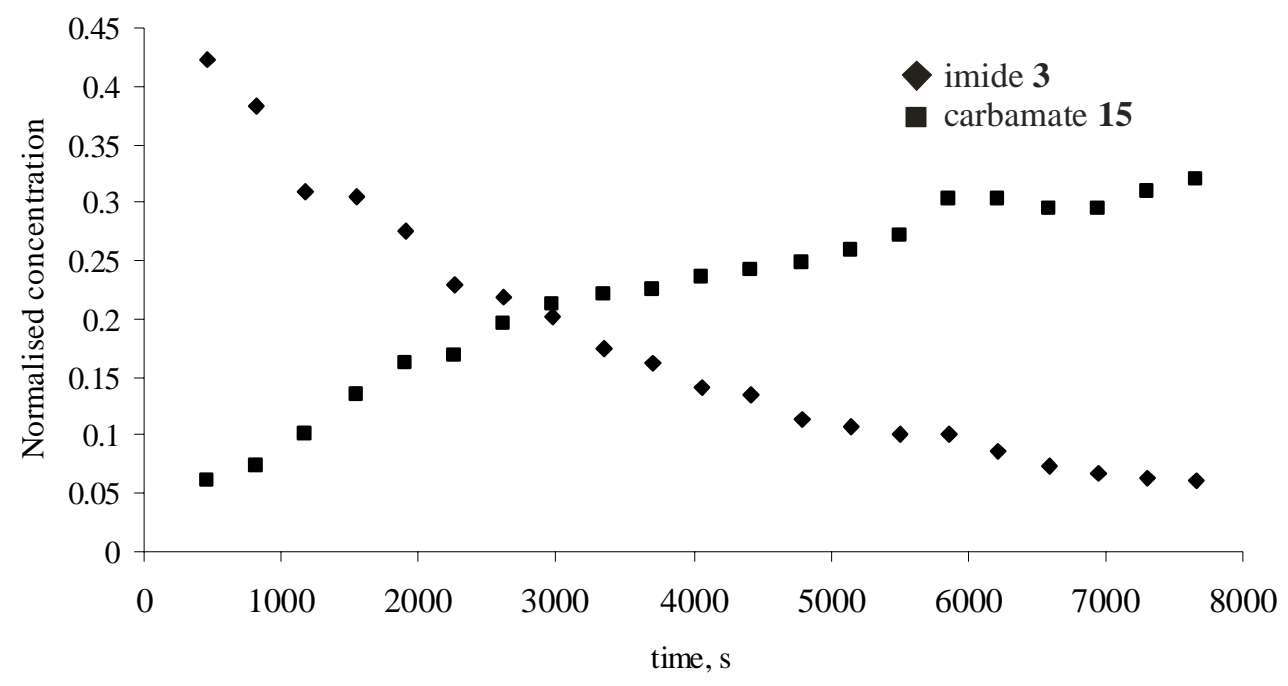

(vi) Plot of $\ln \left(\mathrm{I} / \mathrm{I}_{0}\right) v s$. time for the reaction of 3 with $\mathrm{CO}_{2}(0.75 \mathrm{~atm})$

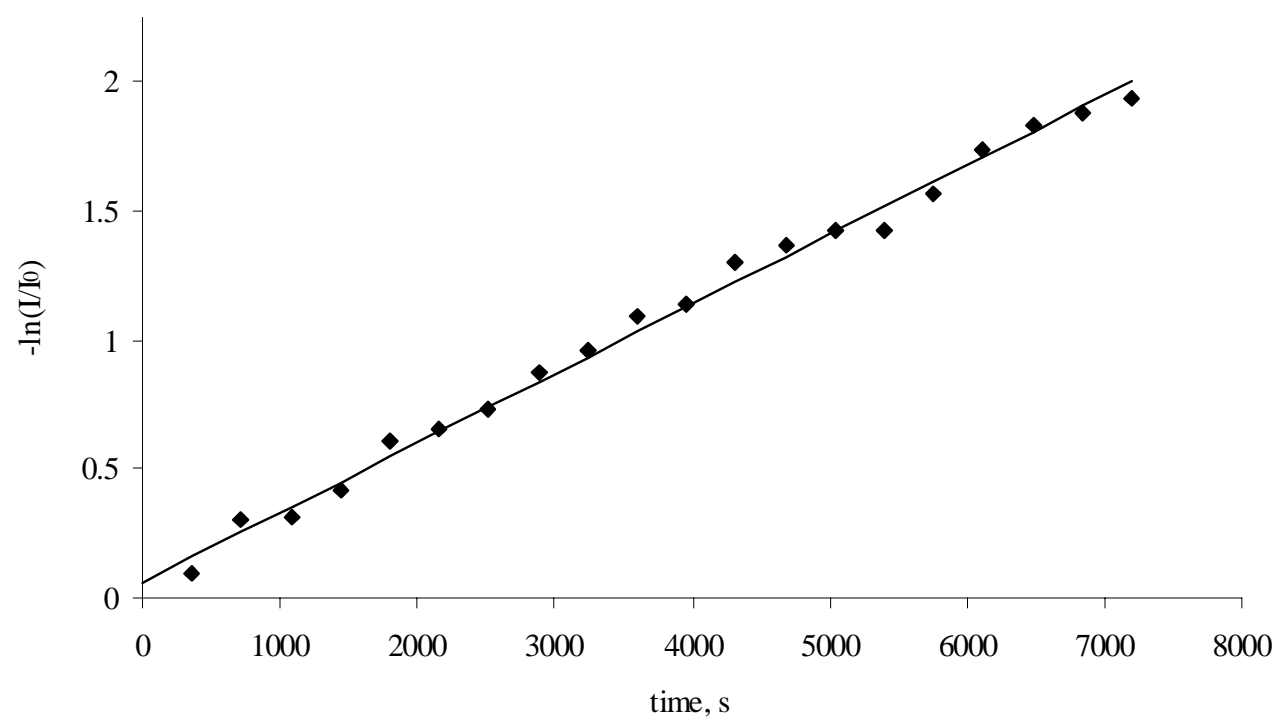


(vii) Plot of normalised concentrations against time for the cycloaddition reaction of $\mathbf{3}$ with $\mathrm{CO}_{2}(1.0 \mathrm{~atm})$. Values are given for starting imide $\mathbf{3}$ and product carbamate $\mathbf{1 5}$

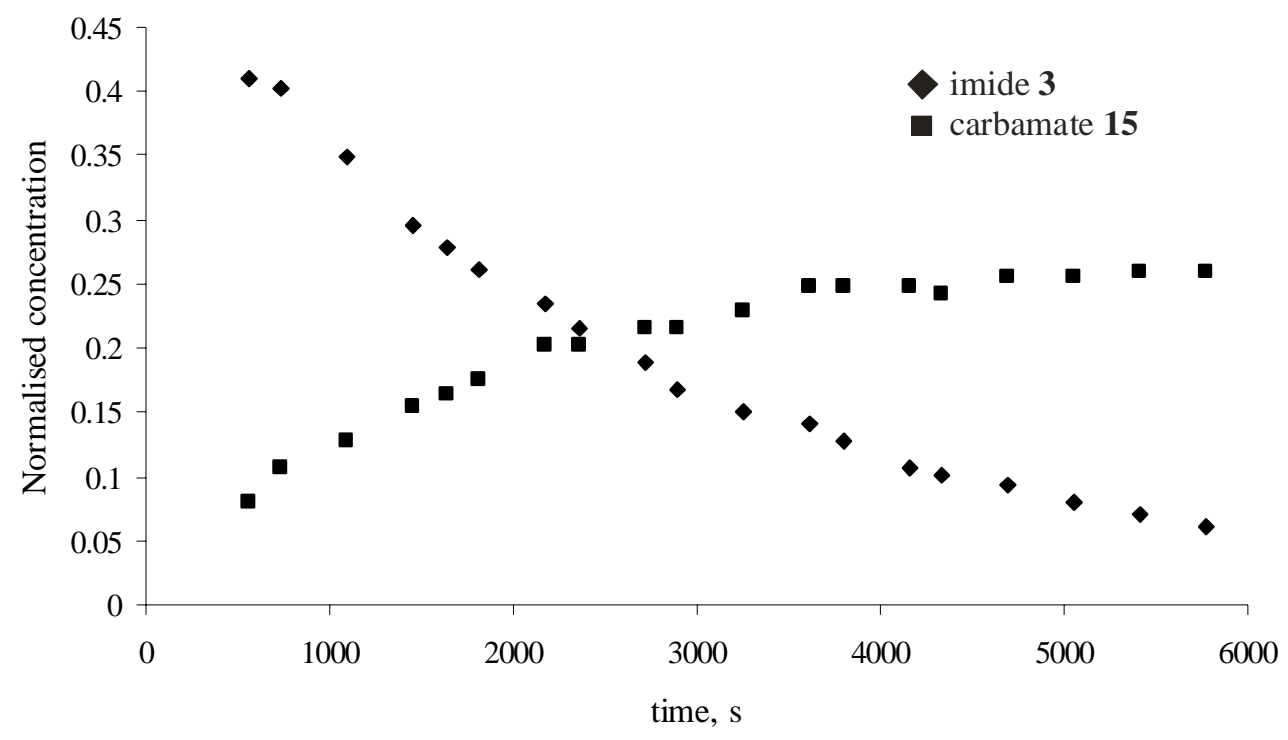

(viii) Plot of $\ln \left(\mathrm{I} / \mathrm{I}_{0}\right) v s$. time for the reaction of $\mathbf{3}$ with $\mathrm{CO}_{2}(1.0 \mathrm{~atm})$

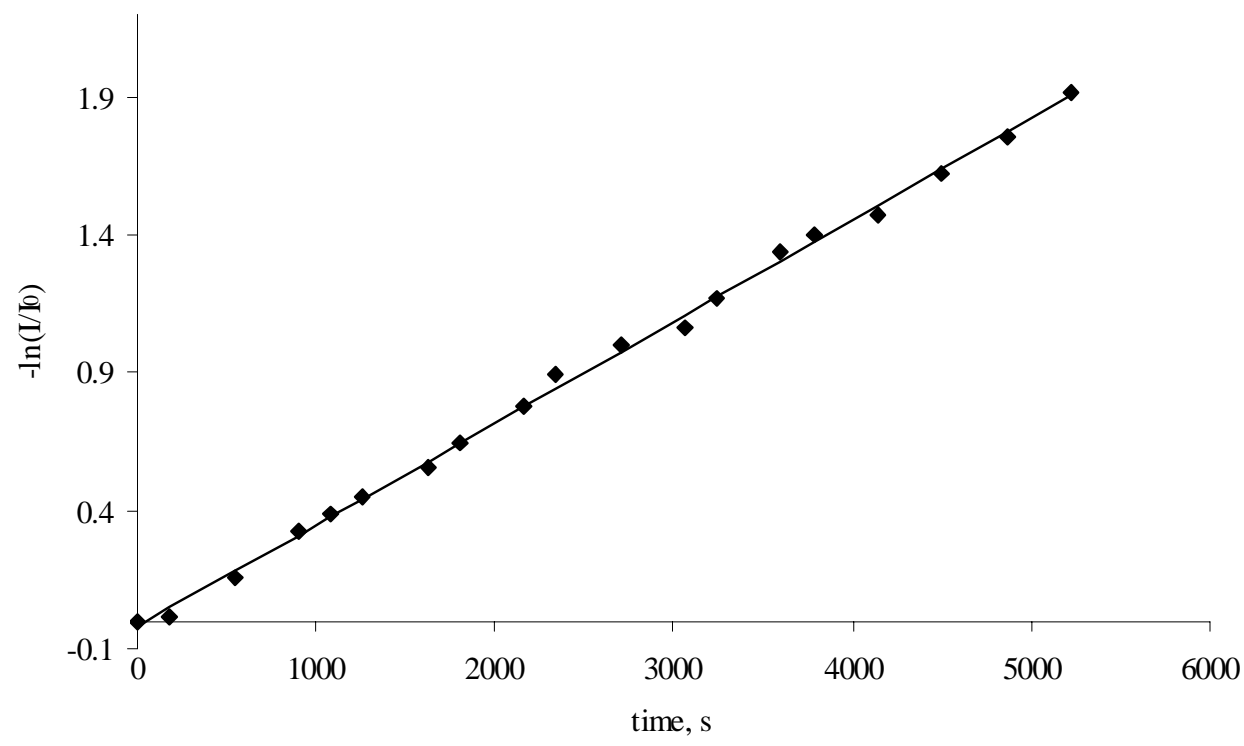




\section{Extrusion of ${ }^{t}$ BuNCO from carbamate intermediates 13, 14 and 15: kinetic studies}

(i) Plot of normalised concentrations for the ${ }^{t} \mathrm{BuNCO}$ extrusion reaction of [Ti( $\eta$ -

$\left.\mathrm{C}_{5} \mathrm{H}_{4} \mathrm{Me}\right)\left\{\mathrm{N}^{t} \mathrm{BuC}(\mathrm{O}) \mathrm{O}\right\}\left\{\mathrm{Me}_{3} \mathrm{SiNC}(\mathrm{Ph}) \mathrm{NCH}_{2} \mathrm{CH}_{2} \mathrm{Me}\right\}(\mathbf{1 4})$ at $1.9 \times 10^{8} \mathrm{~mol} \mathrm{dm}^{-3}$. Values are given for the carbamate $\mathbf{1 4}$ and ${ }^{t} \mathrm{BuNCO}$.

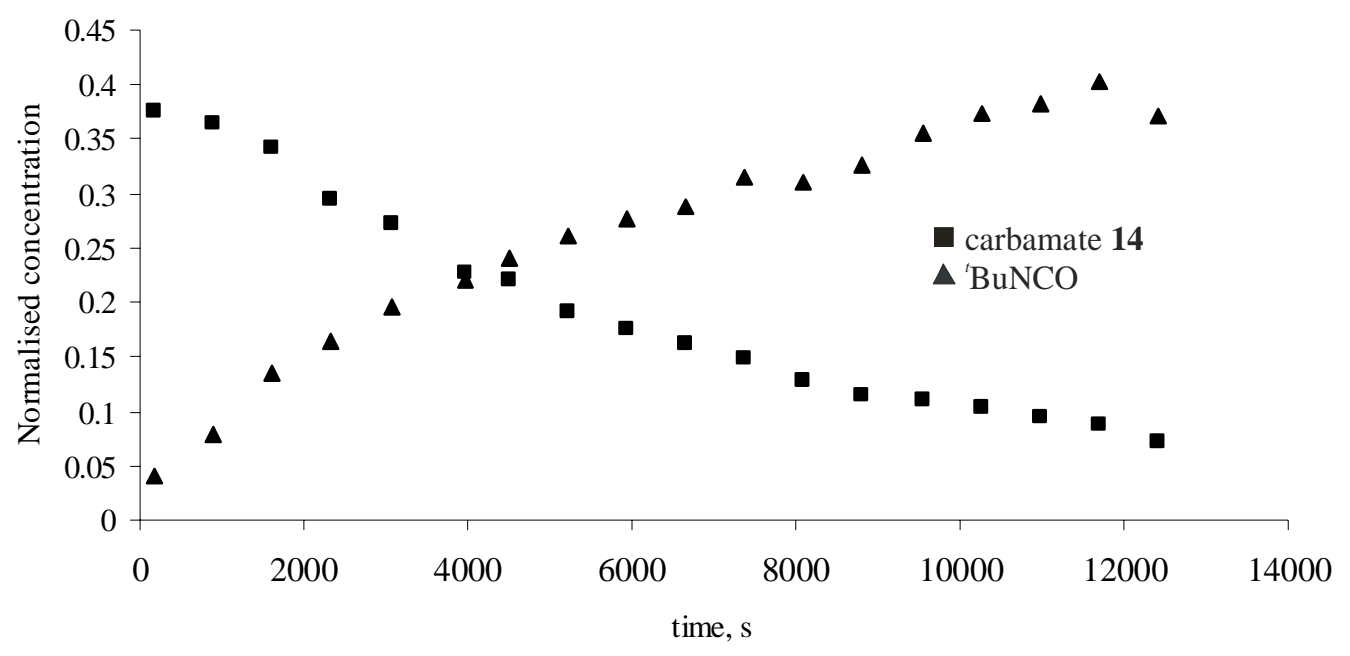

(ii) Plot of $\ln \left(\mathrm{I} / \mathrm{I}_{0}\right) v s$. time for the ${ }^{t} \mathrm{BuNCO}$ extrusion reaction of [Ti( $\eta$ -

$\left.\mathrm{C}_{5} \mathrm{H}_{4} \mathrm{Me}\right)\left\{\mathrm{N}^{t} \mathrm{BuC}(\mathrm{O}) \mathrm{O}\right\}\left\{\mathrm{Me}_{3} \mathrm{SiNC}(\mathrm{Ph}) \mathrm{NCH}_{2} \mathrm{CH}_{2} \mathrm{Me}\right\}(\mathbf{1 4})$ at $1.9 \times 10^{8} \mathrm{~mol} \mathrm{dm}{ }^{-3}$.

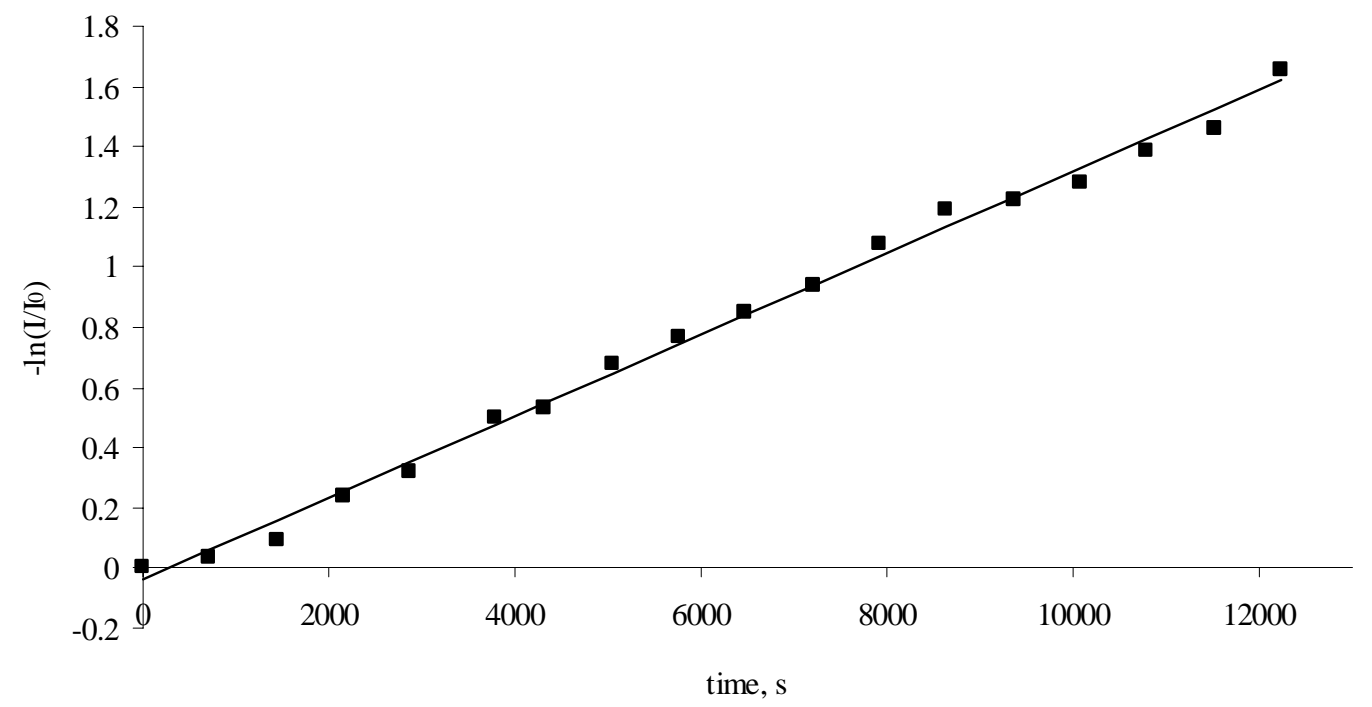


(iii) Plot of normalised concentrations for the ${ }^{t} \mathrm{BuNCO}$ extrusion reaction of [Ti( $\eta$ $\left.\mathrm{C}_{5} \mathrm{H}_{4} \mathrm{Me}\right)\left\{\mathrm{N}^{t} \mathrm{BuC}(\mathrm{O}) \mathrm{O}\right\}\left\{\mathrm{Me}_{3} \mathrm{SiNC}(\mathrm{Ph}) \mathrm{NCH}_{2} \mathrm{CH}_{2} \mathrm{Me}\right\}(\mathbf{1 4})$ at $2.9 \times 10^{8} \mathrm{~mol} \mathrm{dm}^{-3}$. Values are given for the carbamate $\mathbf{1 4}$ and ${ }^{t} \mathrm{BuNCO}$.

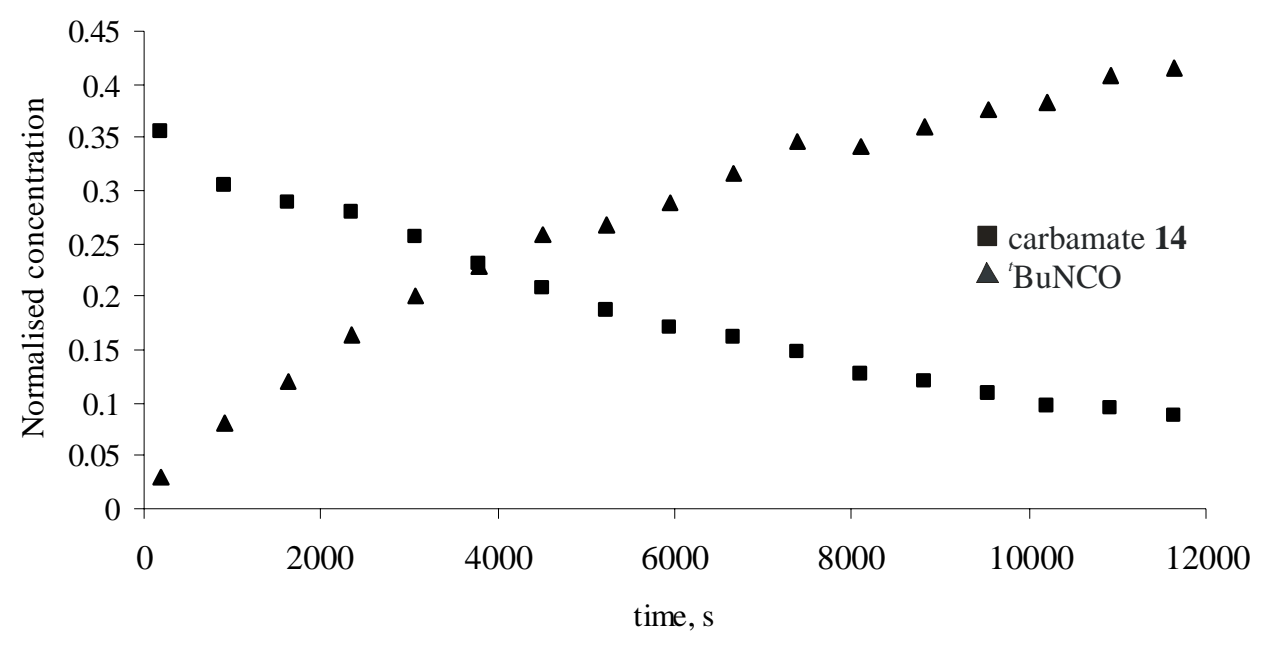

(iv) Plot of $\ln \left(\mathrm{I} / \mathrm{I}_{0}\right) v s$. time for the ${ }^{t} \mathrm{BuNCO}$ extrusion reaction of $[\mathrm{Ti}(\eta-$

$\left.\mathrm{C}_{5} \mathrm{H}_{4} \mathrm{Me}\right)\left\{\mathrm{N}^{t} \mathrm{BuC}(\mathrm{O}) \mathrm{O}\right\}\left\{\mathrm{Me}_{3} \mathrm{SiNC}(\mathrm{Ph}) \mathrm{NCH}_{2} \mathrm{CH}_{2} \mathrm{Me}\right\}(\mathbf{1 4})$ at $2.9 \times 10^{8} \mathrm{~mol} \mathrm{\textrm {dm } ^ { - 3 }}$.

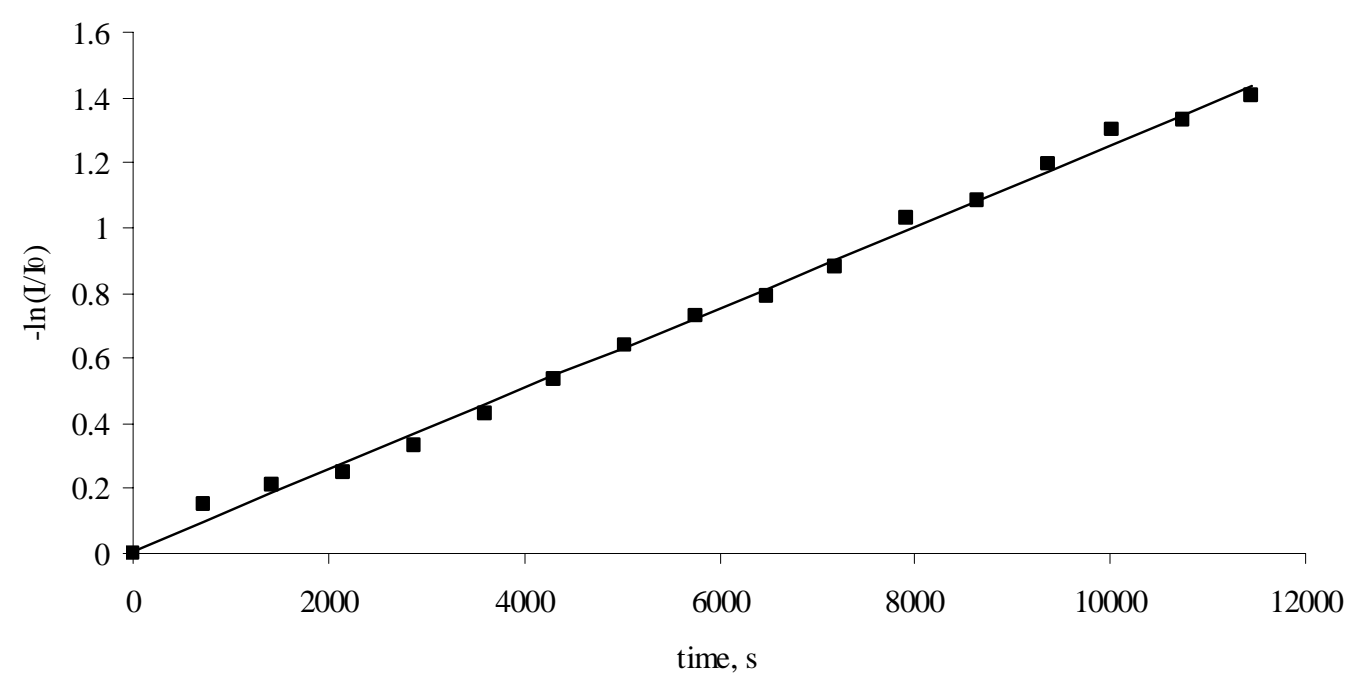


(v) Plot of normalised concentrations for the ${ }^{t} \mathrm{BuNCO}$ extrusion reaction of [Ti( $\eta$ $\left.\mathrm{C}_{5} \mathrm{H}_{4} \mathrm{Me}\right)\left\{\mathrm{N}^{t} \mathrm{BuC}(\mathrm{O}) \mathrm{O}\right\}\left\{\mathrm{Me}_{3} \mathrm{SiNC}(\mathrm{Ph}) \mathrm{NCH}_{2} \mathrm{CH}_{2} \mathrm{Me}\right\}(\mathbf{1 4})$ at $3.8 \times 10^{8} \mathrm{~mol} \mathrm{dm}^{-3}$. Values are given for the carbamate $\mathbf{1 4}$ and ${ }^{t} \mathrm{BuNCO}$.

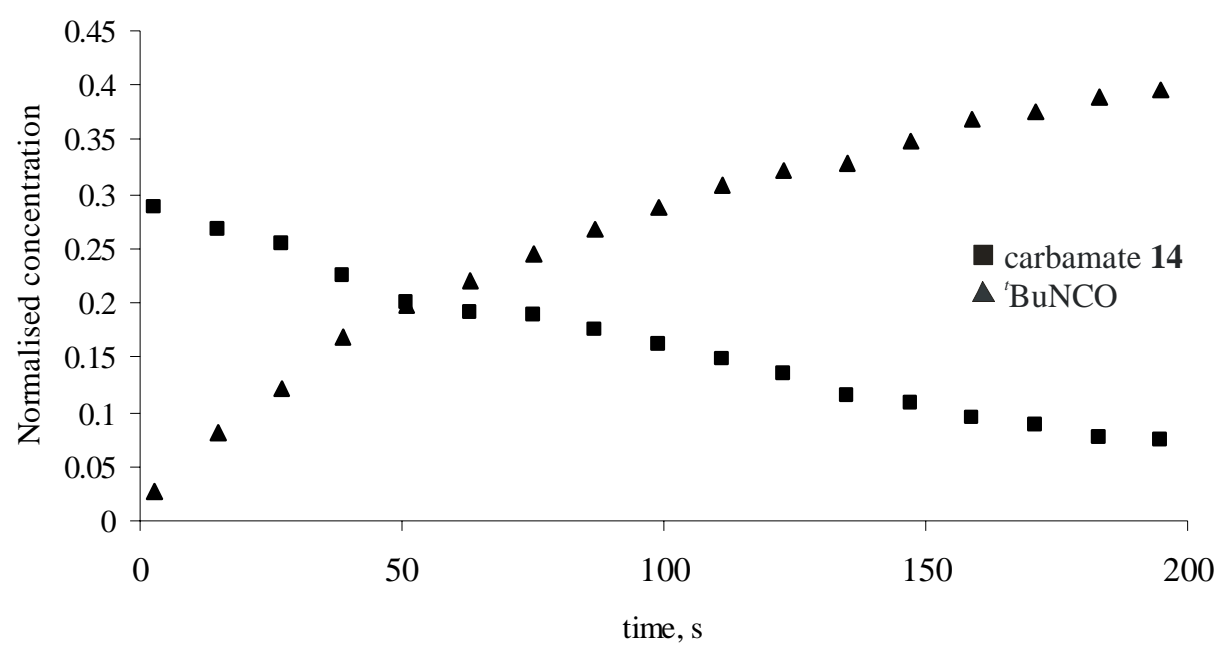

(vi) Plot of $\ln \left(\mathrm{I}_{\mathrm{I}}\right)$ vs. time for the ${ }^{t} \mathrm{BuNCO}$ extrusion reaction of [Ti( $\eta$ -

$\left.\mathrm{C}_{5} \mathrm{H}_{4} \mathrm{Me}\right)\left\{\mathrm{N}^{t} \mathrm{BuC}(\mathrm{O}) \mathrm{O}\right\}\left\{\mathrm{Me}_{3} \mathrm{SiNC}(\mathrm{Ph}) \mathrm{NCH}_{2} \mathrm{CH}_{2} \mathrm{Me}\right\}(\mathbf{1 4})$ at $3.8 \times 10^{8} \mathrm{~mol} \mathrm{dm}{ }^{-3}$.

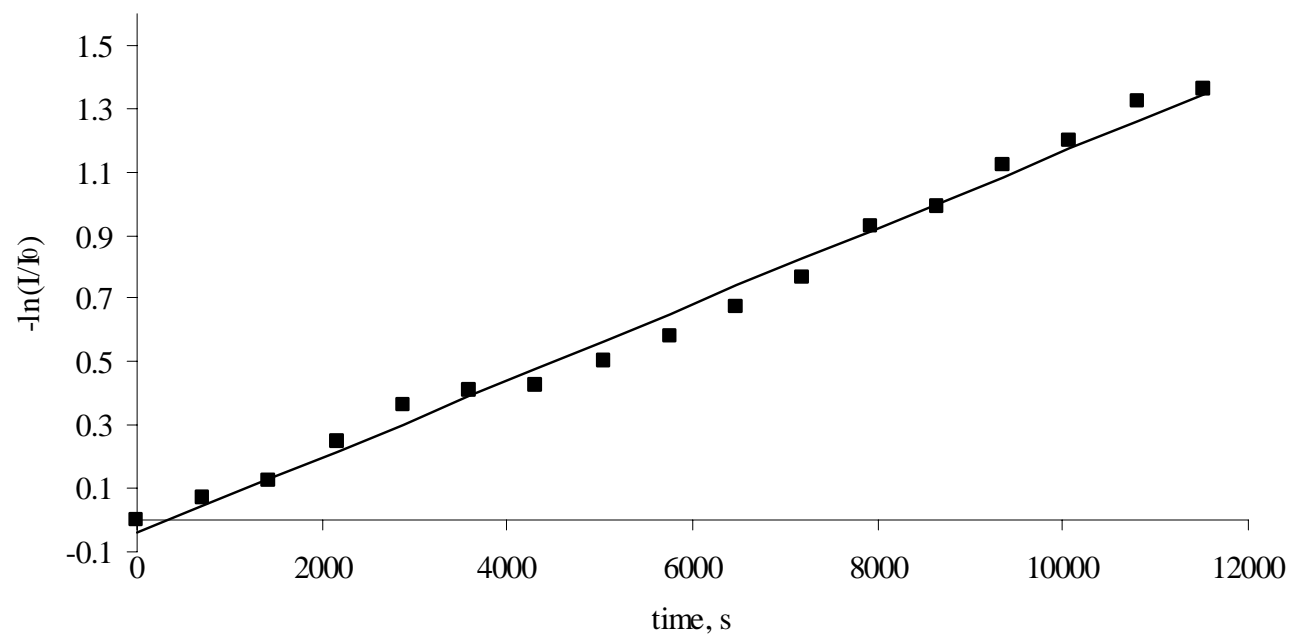


(vii) Plot of normalised concentrations for the ${ }^{t} \mathrm{BuNCO}$ extrusion reaction of [Ti( $\eta$ -

$\left.\mathrm{C}_{5} \mathrm{H}_{4} \mathrm{Me}\right)\left\{\mathrm{N}^{t} \mathrm{BuC}(\mathrm{O}) \mathrm{O}\right\}\left\{\mathrm{Me}_{3} \mathrm{SiNC}(\mathrm{Ph}) \mathrm{NCH}_{2} \mathrm{CH}_{2} \mathrm{NMe}_{2}\right\}(\mathbf{1 5})$ at $1.9 \times 10^{8} \mathrm{~mol} \mathrm{dm}^{-3}$. Values are given for the carbamate $\mathbf{1 5}$ and ${ }^{t} \mathrm{BuNCO}$.

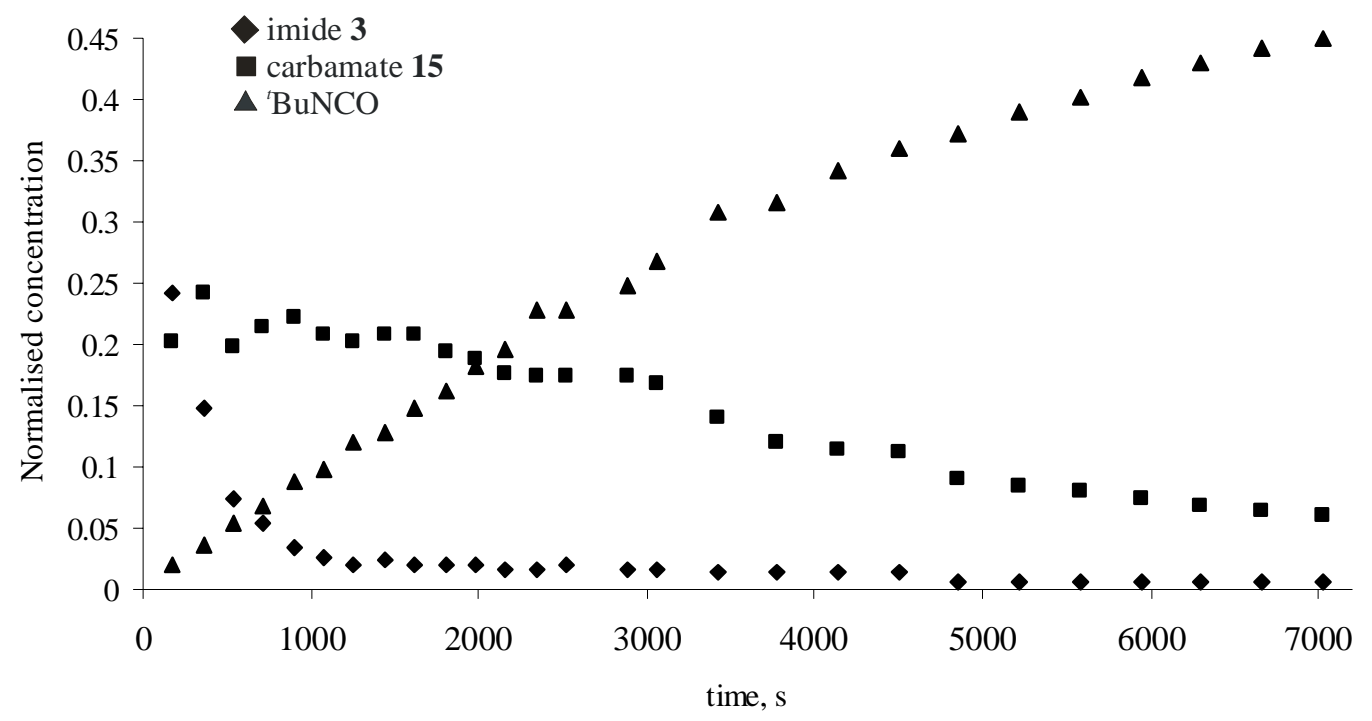

(viii) Plot of $\ln \left(\mathrm{I} / \mathrm{I}_{0}\right) v s$. time for the ${ }^{t} \mathrm{BuNCO}$ extrusion reaction of [Ti( $\eta$ -

$\left.\mathrm{C}_{5} \mathrm{H}_{4} \mathrm{Me}\right)\left\{\mathrm{N}^{t} \mathrm{BuC}(\mathrm{O}) \mathrm{O}\right\}\left\{\mathrm{Me}_{3} \mathrm{SiNC}(\mathrm{Ph}) \mathrm{NCH}_{2} \mathrm{CH}_{2} \mathrm{NMe}_{2}\right\}(\mathbf{1 5})$ at $1.9 \times 10^{8} \mathrm{~mol} \mathrm{dm}^{-3}$.

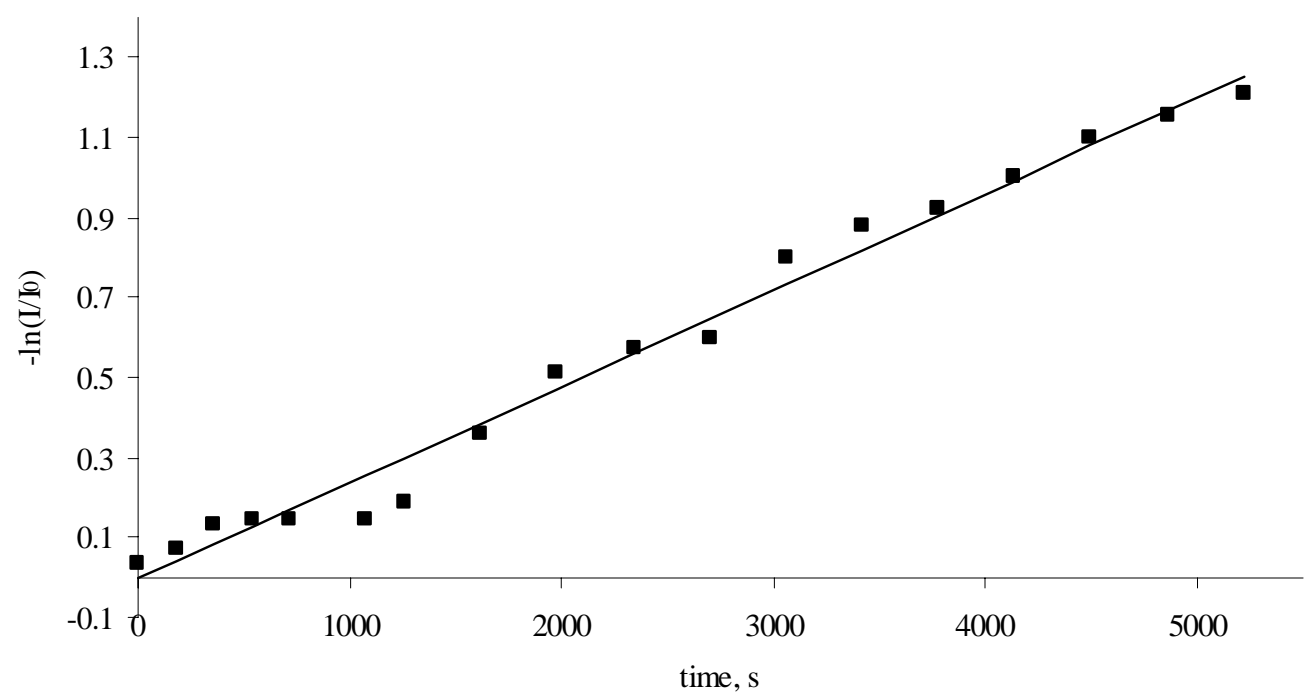


(ix) Plot of normalised concentrations for the ${ }^{t} \mathrm{BuNCO}$ extrusion reaction of [Ti( $\eta$ -

$\left.\mathrm{C}_{5} \mathrm{H}_{4} \mathrm{Me}\right)\left\{\mathrm{N}^{t} \mathrm{BuC}(\mathrm{O}) \mathrm{O}\right\}\left\{\mathrm{Me}_{3} \mathrm{SiNC}(\mathrm{Ph}) \mathrm{NCH}_{2} \mathrm{CH}_{2} \mathrm{NMe}_{2}\right\}(\mathbf{1 5})$ at $1.9 \times 10^{8} \mathrm{~mol} \mathrm{dm}^{-3}$ (duplicate run). Values are given for the carbamate $\mathbf{1 5}$ and ${ }^{t} \mathrm{BuNCO}$.

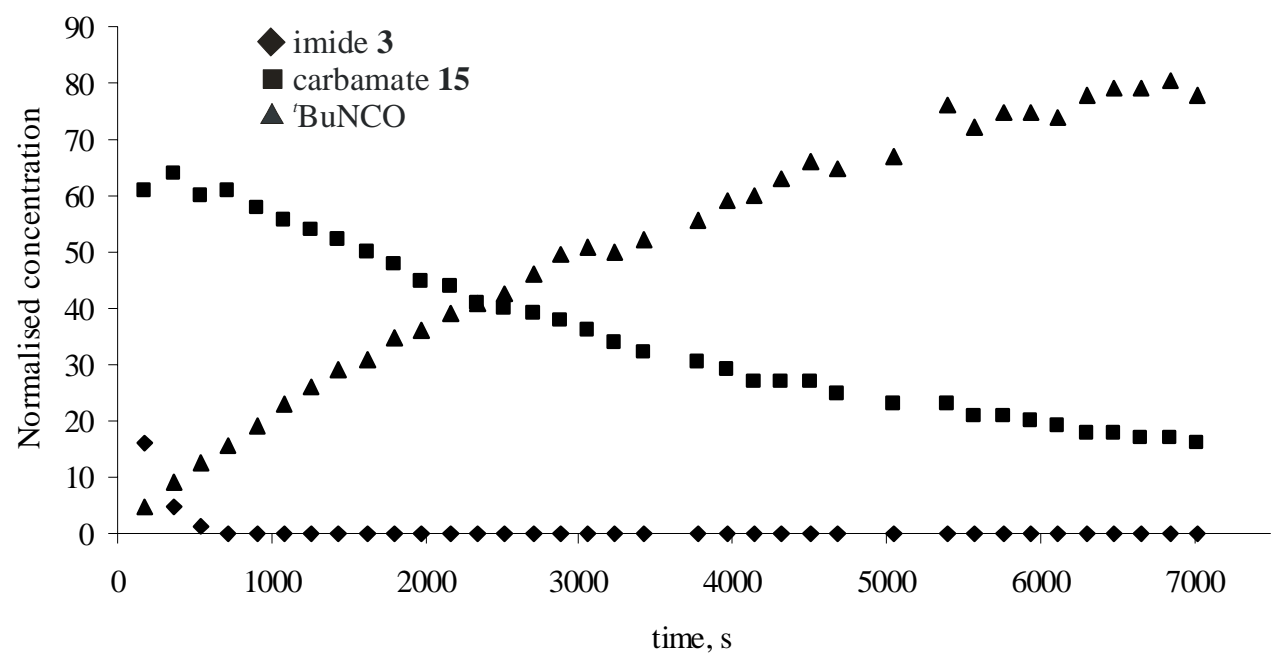

(x) Plot of $\ln \left(\mathrm{I} / \mathrm{I}_{0}\right) v s$. time for the ${ }^{t} \mathrm{BuNCO}$ extrusion reaction of [Ti( $\eta$ -

$\left.\mathrm{C}_{5} \mathrm{H}_{4} \mathrm{Me}\right)\left\{\mathrm{N}^{t} \mathrm{BuC}(\mathrm{O}) \mathrm{O}\right\}\left\{\mathrm{Me}_{3} \mathrm{SiNC}(\mathrm{Ph}) \mathrm{NCH}_{2} \mathrm{CH}_{2} \mathrm{NMe}_{2}\right\}$ (15)at $1.9 \times 10^{8} \mathrm{~mol} \mathrm{dm}^{-3}$ (duplicate run).

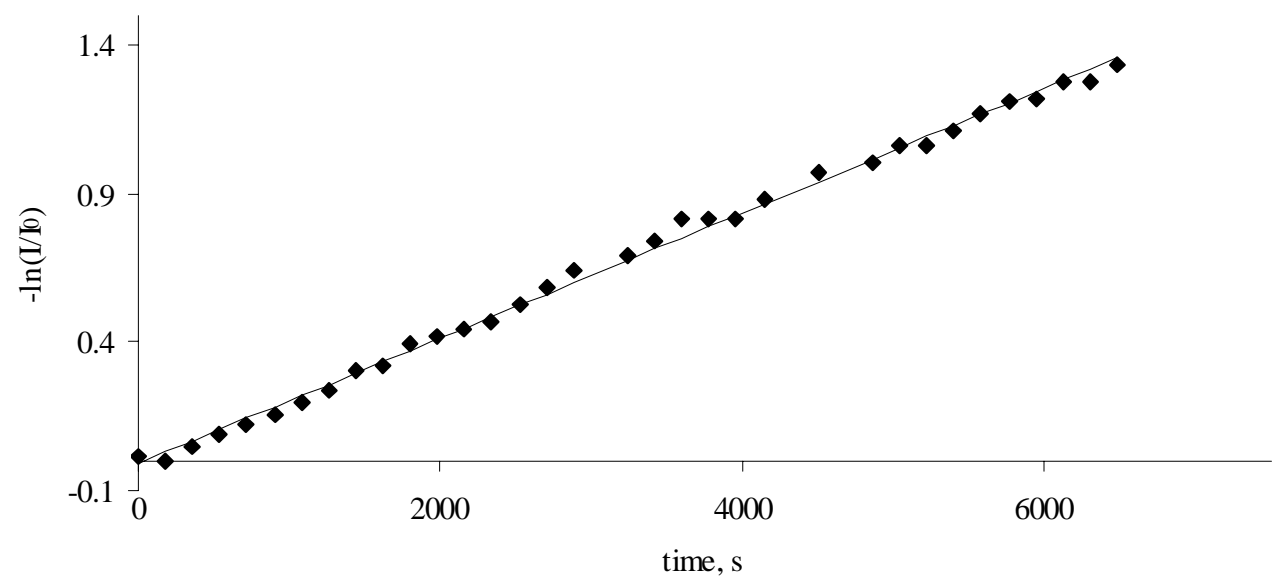


(xi) Plot of normalised concentrations for the ${ }^{t} \mathrm{BuNCO}$ extrusion reaction of [Ti( $\eta$ $\left.\mathrm{C}_{5} \mathrm{H}_{4} \mathrm{Me}\right)\left\{\mathrm{N}^{t} \mathrm{BuC}(\mathrm{O}) \mathrm{O}\right\}\left\{\mathrm{Me}_{3} \mathrm{SiNC}(\mathrm{Ph}) \mathrm{NCH}_{2} \mathrm{CH}_{2} \mathrm{CH}_{2} \mathrm{NMe}_{2}\right\}(\mathbf{1 3})$ at $1.9 \times 10^{8} \mathrm{~mol} \mathrm{dm}^{-3}$. Values are given for the carbamate $\mathbf{1 3}$ and ${ }^{t} \mathrm{BuNCO}$.

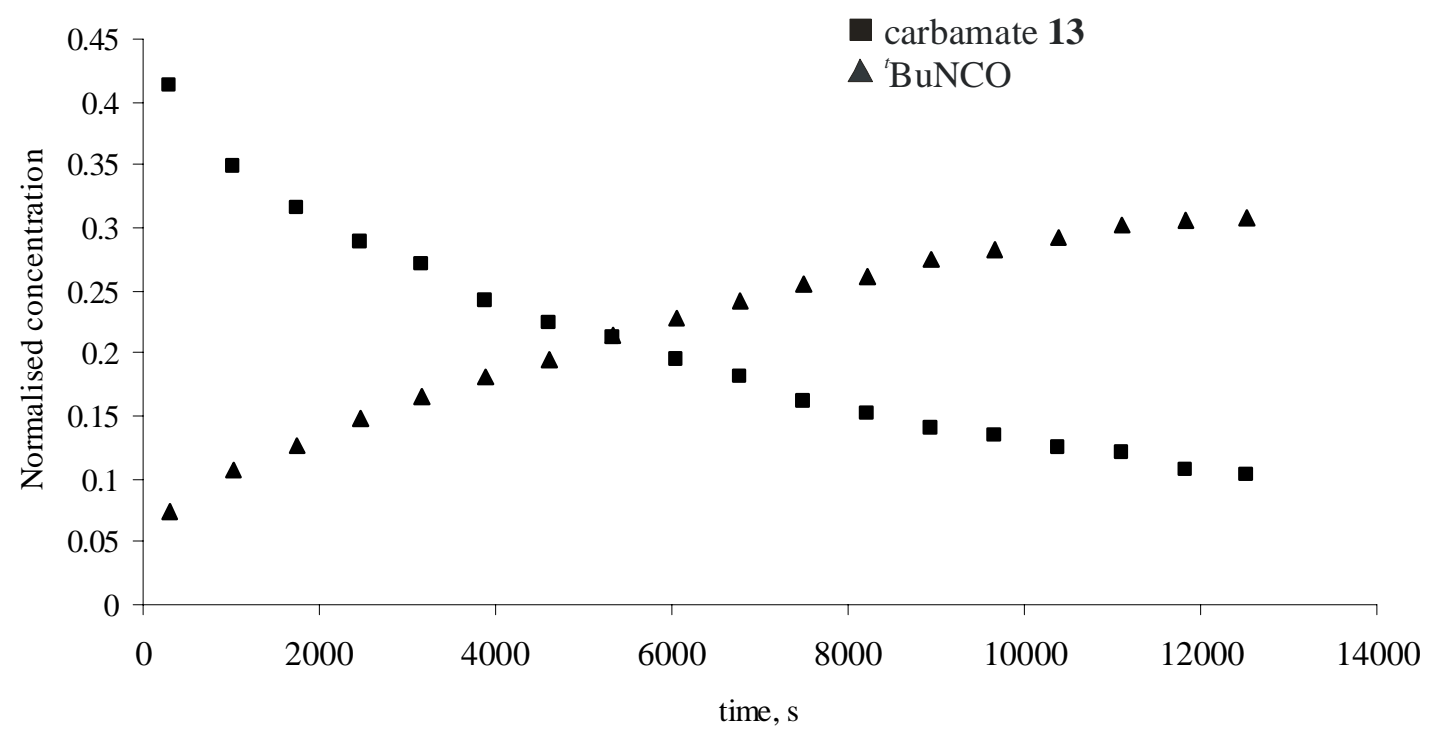

(xii) Plot of $\ln \left(\mathrm{I} / \mathrm{I}_{0}\right)$ vs. time for the ${ }^{t} \mathrm{BuNCO}$ extrusion reaction of $[\mathrm{Ti}(\eta-$

$\left.\mathrm{C}_{5} \mathrm{H}_{4} \mathrm{Me}\right)\left\{\mathrm{N}^{t} \mathrm{BuC}(\mathrm{O}) \mathrm{O}\right\}\left\{\mathrm{Me}_{3} \mathrm{SiNC}(\mathrm{Ph}) \mathrm{NCH}_{2} \mathrm{CH}_{2} \mathrm{CH}_{2} \mathrm{NMe}_{2}\right\}(\mathbf{1 3})$ at $1.9 \times 10^{8} \mathrm{~mol} \mathrm{dm}^{-3}$.

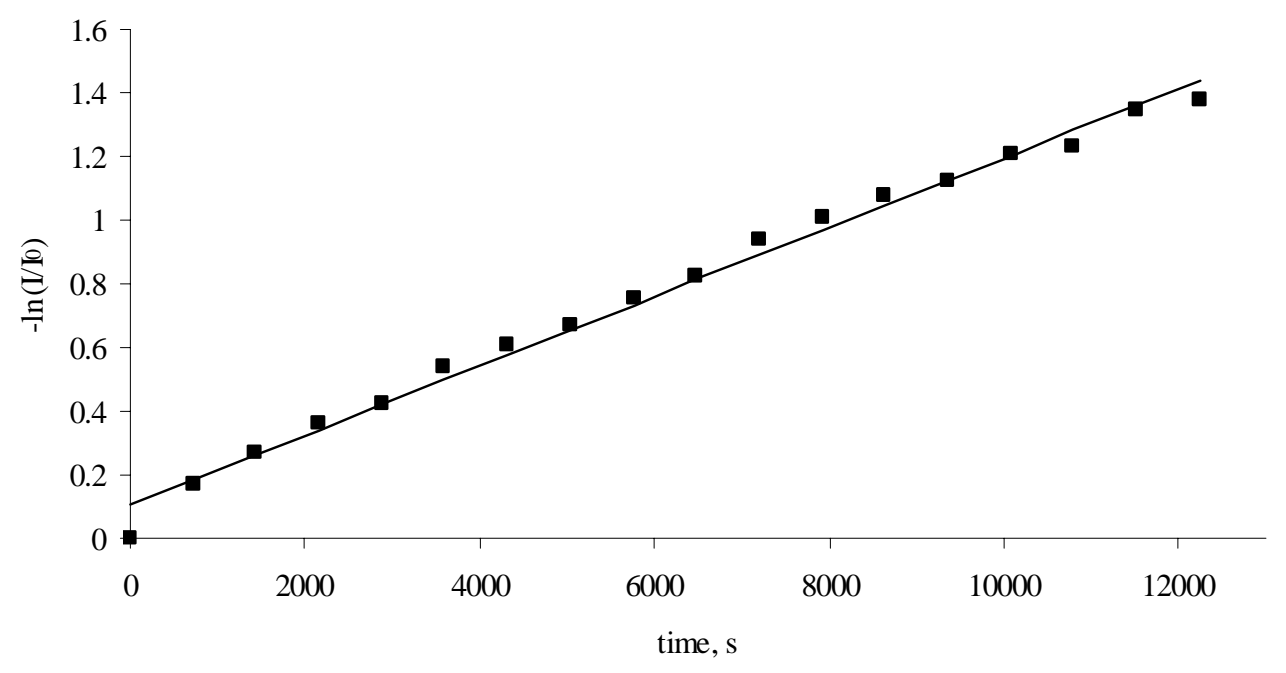


(xiii) Plot of normalised concentrations for the ${ }^{t} \mathrm{BuNCO}$ extrusion reaction of [Ti( $\eta$ $\left.\mathrm{C}_{5} \mathrm{H}_{4} \mathrm{Me}\right)\left\{\mathrm{N}^{t} \mathrm{BuC}(\mathrm{O}) \mathrm{O}\right\}\left\{\mathrm{Me}_{3} \mathrm{SiNC}(\mathrm{Ph}) \mathrm{NCH}_{2} \mathrm{CH}_{2} \mathrm{CH}_{2} \mathrm{NMe}_{2}\right\}(\mathbf{1 3})$ at $1.9 \times 10^{8} \mathrm{~mol} \mathrm{dm}^{-3}$ (duplicate run). Values are given for the carbamate $\mathbf{1 3}$ and ${ }^{t} \mathrm{BuNCO}$

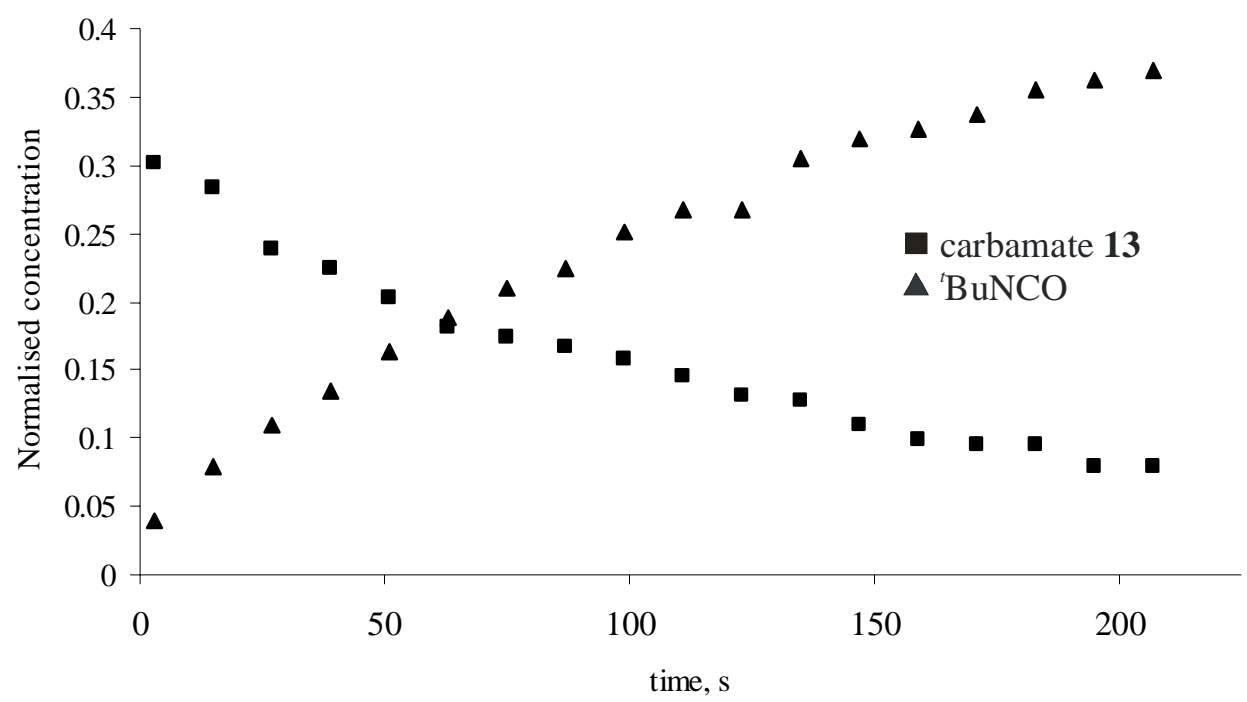

(xiv)

Plot of $\ln \left(\mathrm{I} / \mathrm{I}_{0}\right) v s$. time for the ${ }^{t} \mathrm{BuNCO}$ extrusion reaction of $[\mathrm{Ti}(\eta-$

$\left.\mathrm{C}_{5} \mathrm{H}_{4} \mathrm{Me}\right)\left\{\mathrm{N}^{t} \mathrm{BuC}(\mathrm{O}) \mathrm{O}\right\}\left\{\mathrm{Me}_{3} \mathrm{SiNC}(\mathrm{Ph}) \mathrm{NCH}_{2} \mathrm{CH}_{2} \mathrm{CH}_{2} \mathrm{NMe}_{2}\right\}(\mathbf{1 3})$ at $1.9 \times 10^{8} \mathrm{~mol} \mathrm{dm}^{-3}$ (duplicate run).

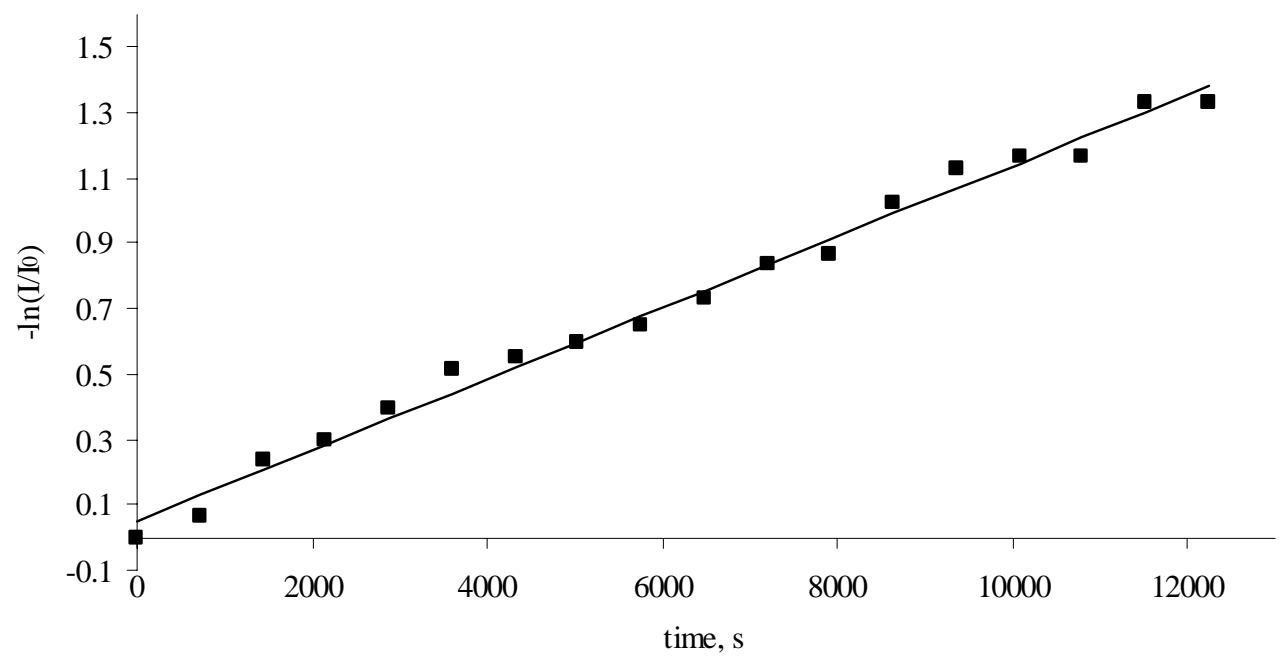




\section{Density functional theory calculations - raw data}

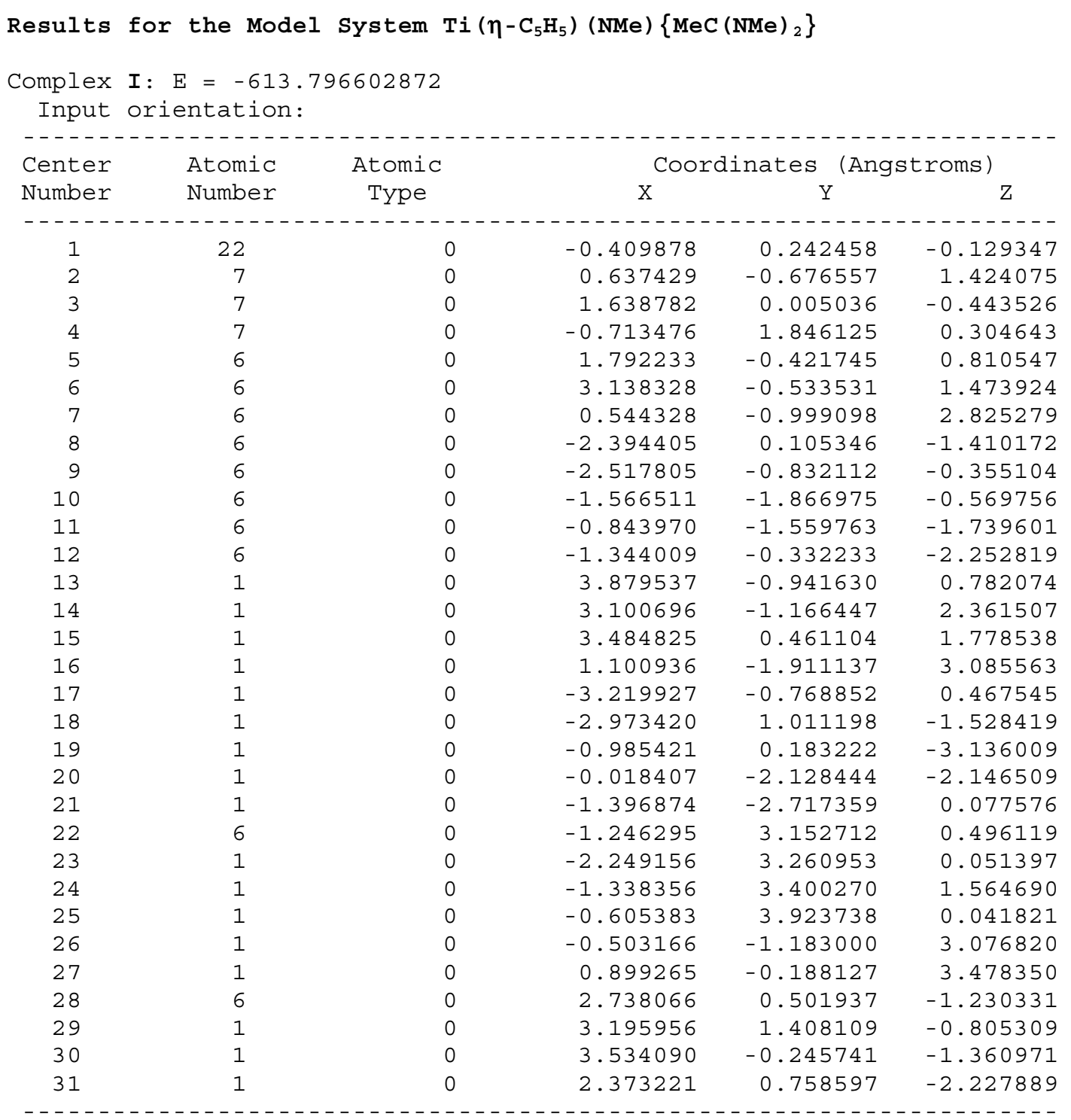

Complex TS I-II $_{\text {I }} \mathrm{E}=-802.300587135$

Input orientation:

\begin{tabular}{|c|c|c|c|c|c|}
\hline \multirow{2}{*}{$\begin{array}{l}\text { Center } \\
\text { Number }\end{array}$} & \multirow{2}{*}{$\begin{array}{l}\text { Atomic } \\
\text { Number }\end{array}$} & \multirow{2}{*}{$\begin{array}{c}\text { Atomic } \\
\text { Type }\end{array}$} & \multicolumn{3}{|c|}{ Coordinates (Angstroms) } \\
\hline & & & $\mathrm{X}$ & $\mathrm{Y}$ & Z \\
\hline 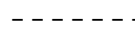 & ---- & -- & ---------- & -------- & -------- \\
\hline 1 & 22 & 0 & -0.310985 & -0.159564 & -0.071586 \\
\hline 2 & 7 & 0 & 0.861660 & -0.723782 & 1.574634 \\
\hline 3 & 7 & 0 & 1.795658 & -0.092975 & -0.313835 \\
\hline 4 & 7 & 0 & -0.988319 & 1.270058 & 0.581692 \\
\hline 5 & 6 & 0 & 1.988553 & -0.358258 & 0.975164 \\
\hline 6 & 6 & 0 & 3.309921 & -0.208010 & 1.680123 \\
\hline 7 & 6 & 0 & 0.689645 & -0.753151 & 3.001724 \\
\hline 8 & 6 & 0 & -2.263121 & -0.958075 & -1.135743 \\
\hline 9 & 6 & 0 & -2.299319 & -1.424923 & 0.202957 \\
\hline 10 & 6 & 0 & -1.241680 & -2.349715 & 0.364777 \\
\hline 11 & 6 & 0 & -0.557537 & -2.469260 & -0.869874 \\
\hline 12 & 6 & 0 & -1.190505 & -1.610970 & -1.795699 \\
\hline
\end{tabular}




$\begin{array}{rrrrrr}13 & 1 & 0 & 4.143363 & -0.331943 & 0.986129 \\ 14 & 1 & 0 & 3.414799 & -0.937983 & 2.485925 \\ 15 & 1 & 0 & 3.386609 & 0.791677 & 2.123699 \\ 16 & 1 & 0 & 1.537195 & -1.221984 & 3.520457 \\ 17 & 1 & 0 & -2.997071 & -1.109056 & 0.967216 \\ 18 & 1 & 0 & -2.937261 & -0.233512 & -1.573778 \\ 19 & 1 & 0 & -0.882568 & -1.443082 & -2.820115 \\ 20 & 1 & 0 & 0.321953 & -3.071700 & -1.054596 \\ 21 & 1 & 0 & -0.975717 & -2.854427 & 1.283695 \\ 22 & 6 & 0 & -1.853090 & 2.192980 & 1.238206 \\ 23 & 1 & 0 & -1.817717 & 3.188873 & 0.773245 \\ 24 & 1 & 0 & -2.903230 & 1.859027 & 1.221666 \\ 25 & 1 & 0 & -1.565776 & 2.320039 & 2.292521 \\ 26 & 1 & 0 & -0.201931 & -1.338858 & 3.245847 \\ 27 & 6 & 0 & -0.476665 & 2.203711 & -1.653318 \\ 28 & 8 & 0 & -0.822362 & 3.304677 & -1.481059 \\ 29 & 8 & 0 & -0.071697 & 1.180291 & -2.099478 \\ 30 & 6 & 0 & 2.860748 & 0.297696 & -1.202298 \\ 31 & 1 & 0 & 3.400388 & 1.195254 & -0.863926 \\ 32 & 1 & 0 & 3.602346 & -0.502273 & -1.347133 \\ 33 & 1 & 0 & 2.436586 & 0.524027 & -2.182946 \\ 34 & 1 & 0 & 0.547488 & 0.251321 & 3.427726 \\ ---1-----19\end{array}$

Complex II: $\mathrm{E}=-802.343891875$

Input orientation:

\begin{tabular}{|c|c|c|c|c|c|}
\hline \multirow{2}{*}{$\begin{array}{l}\text { Center } \\
\text { Number }\end{array}$} & \multirow{2}{*}{$\begin{array}{l}\text { Atomic } \\
\text { Number }\end{array}$} & \multirow{2}{*}{$\begin{array}{l}\text { Atomic } \\
\text { Type }\end{array}$} & \multicolumn{3}{|c|}{ Coordinates (Angstroms) } \\
\hline & & & $\mathrm{X}$ & $\mathrm{Y}$ & Z \\
\hline - - - - - & ----- & ----- & --------1 & --------- & --------- \\
\hline 1 & 22 & 0 & -0.255100 & -0.185267 & -0.286337 \\
\hline 2 & 7 & 0 & 0.622061 & -0.576204 & 1.597850 \\
\hline 3 & 7 & 0 & 1.794542 & -0.282120 & -0.221909 \\
\hline 4 & 7 & 0 & -0.851507 & 1.573088 & 0.185094 \\
\hline 5 & 6 & 0 & 1.835390 & -0.361833 & 1.107567 \\
\hline 6 & 6 & 0 & 3.084435 & -0.174832 & 1.919085 \\
\hline 7 & 6 & 0 & 0.320079 & -0.698515 & 2.999727 \\
\hline 8 & 6 & 0 & -2.377292 & -0.986891 & -0.950344 \\
\hline 9 & 6 & 0 & -2.087406 & -1.614975 & 0.284547 \\
\hline 10 & 6 & 0 & -0.967748 & -2.462262 & 0.091961 \\
\hline 11 & 6 & 0 & -0.561653 & -2.355687 & -1.258581 \\
\hline 12 & 6 & 0 & -1.430686 & -1.439652 & -1.902285 \\
\hline 13 & 1 & 0 & 3.947503 & -0.609826 & 1.409610 \\
\hline 14 & 1 & 0 & 2.990572 & -0.625125 & 2.908891 \\
\hline 15 & 1 & 0 & 3.280276 & 0.895329 & 2.053815 \\
\hline 16 & 1 & 0 & 0.970522 & -1.424015 & 3.507957 \\
\hline 17 & 1 & 0 & -2.611794 & -1.453038 & 1.218008 \\
\hline 18 & 1 & 0 & -3.152900 & -0.253738 & -1.129175 \\
\hline 19 & 1 & 0 & -1.351100 & -1.093838 & -2.923653 \\
\hline 20 & 1 & 0 & 0.286536 & -2.853430 & -1.709943 \\
\hline 21 & 1 & 0 & -0.480459 & -3.052315 & 0.855614 \\
\hline 22 & 6 & 0 & -1.492330 & 2.411295 & 1.166629 \\
\hline 23 & 1 & 0 & -1.162894 & 3.447670 & 1.039492 \\
\hline 24 & 1 & 0 & -2.586338 & 2.395642 & 1.064782 \\
\hline 25 & 1 & 0 & -1.238628 & 2.074019 & 2.175909 \\
\hline 26 & 1 & 0 & -0.708291 & -1.053011 & 3.113383 \\
\hline 27 & 6 & 0 & -0.525307 & 2.078737 & -1.072383 \\
\hline 28 & 8 & 0 & -0.641544 & 3.224164 & -1.458586 \\
\hline 29 & 8 & 0 & -0.052882 & 1.039071 & -1.783911 \\
\hline 30 & 6 & 0 & 2.888068 & 0.173768 & -1.046432 \\
\hline 31 & 1 & 0 & 3.449320 & 0.998858 & -0.588671 \\
\hline
\end{tabular}




$\begin{array}{rrrrrr}32 & 1 & 0 & 3.593472 & -0.638566 & -1.270570 \\ 33 & 1 & 0 & 2.473696 & 0.540091 & -1.988113 \\ 34 & 1 & 0 & 0.399129 & 0.257024 & 3.537863 \\ \ldots\end{array}$

Complex TS II-III $: E=-802.310376194$

Input orientation:

\begin{tabular}{|c|c|c|c|c|c|}
\hline \multirow{2}{*}{$\begin{array}{l}\text { Center } \\
\text { Number }\end{array}$} & \multirow{2}{*}{$\begin{array}{l}\text { Atomic } \\
\text { Number }\end{array}$} & \multirow{2}{*}{$\begin{array}{l}\text { Atomic } \\
\text { Type }\end{array}$} & \multicolumn{3}{|c|}{ Coordinates (Angstroms) } \\
\hline & & & $\mathrm{X}$ & $\mathrm{Y}$ & $\mathrm{Z}$ \\
\hline-- & & & ---- & ------- & ------- \\
\hline 1 & 22 & 0 & -0.394470 & -0.047498 & 0.125341 \\
\hline 2 & 7 & 0 & 0.686764 & -0.842464 & 1.752745 \\
\hline 3 & 7 & 0 & 1.693680 & -0.355404 & -0.129565 \\
\hline 4 & 7 & 0 & -0.135324 & 1.330801 & -1.598020 \\
\hline 5 & 6 & 0 & 1.846737 & -0.569436 & 1.178463 \\
\hline 6 & 6 & 0 & 3.164598 & -0.448973 & 1.892707 \\
\hline 7 & 6 & 0 & 0.467753 & -0.866540 & 3.175361 \\
\hline 8 & 6 & 0 & -2.662676 & -0.544399 & -0.394129 \\
\hline 9 & 6 & 0 & -2.308464 & -1.326088 & 0.729240 \\
\hline 10 & 6 & 0 & -1.373155 & -2.307176 & 0.304168 \\
\hline 11 & 6 & 0 & -1.138456 & -2.127030 & -1.073170 \\
\hline 12 & 6 & 0 & -1.927800 & -1.029445 & -1.502879 \\
\hline 13 & 1 & 0 & 3.959978 & -0.937289 & 1.323110 \\
\hline 14 & 1 & 0 & 3.128286 & -0.888413 & 2.890145 \\
\hline 15 & 1 & 0 & 3.435192 & 0.608131 & 1.995846 \\
\hline 16 & 1 & 0 & 1.240370 & -1.435306 & 3.709977 \\
\hline 17 & 1 & 0 & -2.677383 & -1.188170 & 1.737483 \\
\hline 18 & 1 & 0 & -3.339654 & 0.299340 & -0.393841 \\
\hline 19 & 1 & 0 & -1.972097 & -0.633984 & -2.509458 \\
\hline 20 & 1 & 0 & -0.447847 & -2.696595 & -1.681639 \\
\hline 21 & 1 & 0 & -0.881137 & -3.028928 & 0.941539 \\
\hline 22 & 6 & 0 & 0.175088 & 1.280095 & -3.028017 \\
\hline 23 & 1 & 0 & 0.363939 & 0.239648 & -3.294205 \\
\hline 24 & 1 & 0 & -0.663019 & 1.646988 & -3.630117 \\
\hline 25 & 1 & 0 & 1.065120 & 1.871152 & -3.266018 \\
\hline 26 & 1 & 0 & -0.489346 & -1.355503 & 3.380286 \\
\hline 27 & 6 & 0 & -0.419492 & 2.419700 & -1.044072 \\
\hline 28 & 8 & 0 & -0.635094 & 3.545234 & -0.813862 \\
\hline 29 & 8 & 0 & -0.764377 & 1.413097 & 0.836528 \\
\hline 30 & 6 & 0 & 2.795689 & 0.065443 & -0.957175 \\
\hline 31 & 1 & 0 & 3.177278 & 1.066618 & -0.702102 \\
\hline 32 & 1 & 0 & 3.643391 & -0.632945 & -0.906687 \\
\hline 33 & 1 & 0 & 2.475978 & 0.093636 & -2.000987 \\
\hline 34 & 1 & 0 & 0.420278 & 0.142503 & 3.608741 \\
\hline
\end{tabular}

Complex III: $\mathrm{E}=-802.312115780$

Input orientation:

\begin{tabular}{|c|c|c|c|c|c|}
\hline \multirow{2}{*}{$\begin{array}{l}\text { Center } \\
\text { Number }\end{array}$} & \multirow{2}{*}{$\begin{array}{l}\text { Atomic } \\
\text { Number }\end{array}$} & \multirow{2}{*}{$\begin{array}{c}\text { Atomic } \\
\text { Type }\end{array}$} & \multicolumn{3}{|c|}{ Coordinates (Angstroms) } \\
\hline & & & $\mathrm{X}$ & $\mathrm{Y}$ & Z \\
\hline--- & & & ------1 & ------- & ------- \\
\hline 1 & 22 & 0 & 0.370099 & 0.248497 & 0.088120 \\
\hline 2 & 7 & 0 & 0.441521 & 0.296663 & -2.017866 \\
\hline 3 & 7 & 0 & -1.169014 & -0.787081 & -0.985300 \\
\hline 4 & 7 & 0 & -1.109842 & -0.240462 & 1.851870 \\
\hline 5 & 6 & 0 & -0.780961 & -0.196607 & -2.119442 \\
\hline 6 & 6 & 0 & -1.669502 & -0.064047 & -3.326503 \\
\hline 7 & 6 & 0 & 1.036235 & 1.181237 & -2.985559 \\
\hline 8 & 6 & 0 & 2.378721 & 0.522288 & 1.377147 \\
\hline
\end{tabular}




\begin{tabular}{|c|c|c|c|c|c|}
\hline 9 & 6 & 0 & 2.770666 & 0.327706 & 0.033928 \\
\hline 10 & 6 & 0 & 2.489519 & -1.021726 & -0.319466 \\
\hline 11 & 6 & 0 & 1.895556 & -1.649416 & 0.787669 \\
\hline 12 & 6 & 0 & 1.811213 & -0.687181 & 1.834755 \\
\hline 13 & 1 & 0 & -2.150326 & -1.018030 & -3.560357 \\
\hline 14 & 1 & 0 & -1.117514 & 0.273362 & -4.204087 \\
\hline 15 & 1 & 0 & -2.464226 & 0.662824 & -3.123351 \\
\hline 16 & 1 & 0 & 0.932465 & 0.810650 & -4.014300 \\
\hline 17 & 1 & 0 & 3.217570 & 1.076090 & -0.607797 \\
\hline 18 & 1 & 0 & 2.441643 & 1.448742 & 1.931396 \\
\hline 19 & 1 & 0 & 1.419092 & -0.858783 & 2.829010 \\
\hline 20 & 1 & 0 & 1.545630 & -2.673556 & 0.826931 \\
\hline 21 & 1 & 0 & 2.643972 & -1.464146 & -1.294371 \\
\hline 22 & 6 & 0 & -1.357425 & -1.536083 & 2.485323 \\
\hline 23 & 1 & 0 & -0.981518 & -2.311505 & 1.818088 \\
\hline 24 & 1 & 0 & -0.823778 & -1.605003 & 3.438063 \\
\hline 25 & 1 & 0 & -2.424105 & -1.701287 & 2.661972 \\
\hline 26 & 1 & 0 & 2.107625 & 1.262280 & -2.782253 \\
\hline 27 & 6 & 0 & -1.656715 & 0.815040 & 2.180940 \\
\hline 28 & 8 & 0 & -2.175876 & 1.809233 & 2.494768 \\
\hline 29 & 8 & 0 & -0.052369 & 1.806257 & 0.348693 \\
\hline 30 & 6 & 0 & -2.531267 & -1.234073 & -0.828942 \\
\hline 31 & 1 & 0 & -3.264131 & -0.411265 & -0.827239 \\
\hline 32 & 1 & 0 & -2.830653 & -1.939174 & -1.617410 \\
\hline 33 & 1 & 0 & -2.638742 & -1.764837 & 0.119394 \\
\hline 34 & 1 & 0 & 0.615899 & 2.196118 & -2.940037 \\
\hline
\end{tabular}

Complex IV: $E=-594.403261996$

Input orientation:

\begin{tabular}{|c|c|c|c|c|c|}
\hline \multirow{2}{*}{$\begin{array}{l}\text { Center } \\
\text { Number }\end{array}$} & \multirow{2}{*}{$\begin{array}{l}\text { Atomic } \\
\text { Number }\end{array}$} & \multirow{2}{*}{$\begin{array}{l}\text { Atomic } \\
\text { Type }\end{array}$} & \multicolumn{3}{|c|}{ Coordinates (Angstroms) } \\
\hline & & & $\mathrm{X}$ & $\mathrm{Y}$ & $\mathrm{Z}$ \\
\hline--- & ---1 & $\ldots$ & -------- & -------- & -------- \\
\hline 1 & 22 & 0 & 0.321736 & 0.517440 & 0.329910 \\
\hline 2 & 7 & 0 & -0.697905 & 0.149276 & -1.434185 \\
\hline 3 & 7 & 0 & -1.406715 & -0.597179 & 0.536168 \\
\hline 4 & 6 & 0 & -1.717336 & -0.354307 & -0.739613 \\
\hline 5 & 6 & 0 & -3.095436 & -0.571386 & -1.299524 \\
\hline 6 & 6 & 0 & -0.814568 & 0.617775 & -2.793290 \\
\hline 7 & 6 & 0 & 2.617881 & 0.690778 & 0.863763 \\
\hline 8 & 6 & 0 & 2.576225 & 0.104207 & -0.425146 \\
\hline 9 & 6 & 0 & 2.035316 & -1.204570 & -0.302504 \\
\hline 10 & 6 & 0 & 1.703047 & -1.410264 & 1.049312 \\
\hline 11 & 6 & 0 & 2.048917 & -0.232519 & 1.772751 \\
\hline 12 & 1 & 0 & -3.517918 & -1.511064 & -0.934918 \\
\hline 13 & 1 & 0 & -3.091267 & -0.585671 & -2.389868 \\
\hline 14 & 1 & 0 & -3.757037 & 0.238099 & -0.970233 \\
\hline 15 & 1 & 0 & -1.155517 & -0.171187 & -3.477900 \\
\hline 16 & 1 & 0 & 2.915716 & 0.569233 & -1.343332 \\
\hline 17 & 1 & 0 & 2.957983 & 1.689240 & 1.102456 \\
\hline 18 & 1 & 0 & 1.912173 & -0.070067 & 2.835207 \\
\hline 19 & 1 & 0 & 1.232388 & -2.293283 & 1.462104 \\
\hline 20 & 1 & 0 & 1.850513 & -1.893235 & -1.116494 \\
\hline 21 & 1 & 0 & 0.170491 & 0.938770 & -3.140072 \\
\hline 22 & 8 & 0 & -0.013045 & 2.028595 & 0.819047 \\
\hline 23 & 6 & 0 & -2.400435 & -0.934072 & 1.525928 \\
\hline 24 & 1 & 0 & -3.113481 & -0.117061 & 1.708728 \\
\hline 25 & 1 & 0 & -2.973731 & -1.830221 & 1.250779 \\
\hline 26 & 1 & 0 & -1.899054 & -1.150276 & 2.472174 \\
\hline 27 & 1 & 0 & -1.494413 & 1.475747 & -2.892958 \\
\hline
\end{tabular}


Results for the Model System $\mathrm{Ti}\left(\eta-\mathrm{C}_{5} \mathrm{H}_{5}\right)(\mathrm{NMe})\left\{\mathrm{MeC}\left(\mathrm{NCH}_{2} \mathrm{CH}_{2} \mathrm{NMe}_{2}\right)\left(\mathrm{NSiH}_{3}\right)\right\}$

$\underline{\mathrm{CO}}_{2}$ attack from $\mathrm{SiH}_{3}$ side

Complex I : $E=-752.720434271$

Input orientation:

\begin{tabular}{|c|c|c|c|c|c|}
\hline \multirow{2}{*}{$\begin{array}{l}\text { Center } \\
\text { Number }\end{array}$} & \multirow{2}{*}{$\begin{array}{l}\text { Atomic } \\
\text { Number }\end{array}$} & \multirow{2}{*}{$\begin{array}{l}\text { Atomic } \\
\text { Type }\end{array}$} & \multicolumn{3}{|c|}{ Coordinates (Angstroms) } \\
\hline & & & $\mathrm{X}$ & $\mathrm{Y}$ & Z \\
\hline-- & & & -------- & --------- & -------- \\
\hline 1 & 22 & 0 & .024773 & .530691 & -1.136998 \\
\hline 2 & 7 & 0 & .293802 & -.763594 & .495852 \\
\hline 3 & 7 & 0 & 1.954722 & .504162 & -.297952 \\
\hline 4 & 7 & 0 & -.806304 & 1.890882 & -.589612 \\
\hline 5 & 6 & 0 & 1.542765 & -.353864 & .664898 \\
\hline 6 & 6 & 0 & 2.443412 & -.745813 & 1.808607 \\
\hline 7 & 6 & 0 & -.443046 & -1.592454 & 1.434439 \\
\hline 8 & 6 & 0 & -1.206390 & -.742207 & 2.463319 \\
\hline 9 & 14 & 0 & 3.457482 & 1.384040 & -.376074 \\
\hline 10 & 6 & 0 & -1.054610 & .570259 & -3.237023 \\
\hline 11 & 6 & 0 & -1.357662 & -.680477 & -2.646026 \\
\hline 12 & 6 & 0 & -.157082 & -1.439798 & -2.574962 \\
\hline 13 & 6 & 0 & .887972 & -.652239 & -3.098081 \\
\hline 14 & 6 & 0 & .337892 & .597523 & -3.494112 \\
\hline 15 & 1 & 0 & 3.434869 & -1.013276 & 1.427920 \\
\hline 16 & 1 & 0 & 2.057667 & -1.587828 & 2.385233 \\
\hline 17 & 1 & 0 & 2.568648 & .104535 & 2.490078 \\
\hline 18 & 1 & 0 & .210390 & -2.292645 & 1.970464 \\
\hline 19 & 1 & 0 & -1.831711 & .000579 & 1.931663 \\
\hline 20 & 1 & 0 & -.470737 & -.176005 & 3.047841 \\
\hline 21 & 1 & 0 & -2.337139 & -.999415 & -2.307860 \\
\hline 22 & 1 & 0 & -1.752979 & 1.376552 & -3.419339 \\
\hline 23 & 1 & 0 & .888103 & 1.430846 & -3.916733 \\
\hline 24 & 1 & 0 & 1.933026 & -.930895 & -3.147429 \\
\hline 25 & 1 & 0 & -.057623 & -2.432819 & -2.154584 \\
\hline 26 & 6 & 0 & -1.635056 & 3.047821 & -.409291 \\
\hline 27 & 1 & 0 & -2.257625 & 3.248890 & -1.295874 \\
\hline 28 & 1 & 0 & -2.313245 & 2.924299 & .448621 \\
\hline 29 & 1 & 0 & -1.029898 & 3.946927 & -.220830 \\
\hline 30 & 1 & 0 & -1.145237 & -2.200133 & .852438 \\
\hline 31 & 1 & 0 & 4.653716 & .471099 & -.443214 \\
\hline 32 & 1 & 0 & 3.672750 & 2.294672 & .802956 \\
\hline 33 & 1 & 0 & 3.420976 & 2.209834 & -1.622441 \\
\hline 34 & 7 & 0 & -1.985989 & -1.568020 & 3.392710 \\
\hline 35 & 6 & 0 & -2.310026 & -.823290 & 4.610216 \\
\hline 36 & 1 & 0 & -1.386812 & -.497371 & 5.100683 \\
\hline 37 & 1 & 0 & -2.936831 & .070305 & 4.421859 \\
\hline 38 & 1 & 0 & -2.850997 & -1.476879 & 5.303143 \\
\hline 39 & 6 & 0 & -3.213347 & -2.071549 & 2.773908 \\
\hline 40 & 1 & 0 & -3.740122 & -2.716841 & 3.484931 \\
\hline 41 & 1 & 0 & -3.902215 & -1.260082 & 2.465761 \\
\hline 42 & 1 & 0 & -2.979276 & -2.673580 & 1.891366 \\
\hline
\end{tabular}

Complex TS $_{\text {I-II }}: E=-941.223325193$

Input orientation:

Center Atomic Atomic Coordinates (Angstroms) 


\begin{tabular}{|c|c|c|c|c|c|}
\hline Number & Number & Type & $\mathrm{x}$ & $\mathrm{Y}$ & Z \\
\hline-- & -- & --- & ------- & -------- & ------ \\
\hline 1 & 22 & 0 & -0.931833 & -0.438271 & -0.226777 \\
\hline 2 & 7 & 0 & 0.802040 & 0.663250 & -0.708337 \\
\hline 3 & 7 & 0 & -1.065712 & 1.703004 & -0.150640 \\
\hline 4 & 7 & 0 & -0.388075 & -1.198235 & 1.205421 \\
\hline 5 & 6 & 0 & 0.246819 & 1.832053 & -0.447414 \\
\hline 6 & 6 & 0 & 0.961776 & 3.158868 & -0.455921 \\
\hline 7 & 6 & 0 & 2.215436 & 0.445587 & -0.955756 \\
\hline 8 & 6 & 0 & 3.005688 & 0.233384 & 0.345878 \\
\hline 9 & 14 & 0 & -2.227750 & 2.944602 & 0.234397 \\
\hline 10 & 6 & 0 & -1.842028 & -2.427691 & -1.114109 \\
\hline 11 & 6 & 0 & -0.547793 & -2.268809 & -1.670105 \\
\hline 12 & 6 & 0 & -0.576717 & -1.131918 & -2.517199 \\
\hline 13 & 6 & 0 & -1.878479 & -0.583170 & -2.483179 \\
\hline 14 & 6 & 0 & -2.658705 & -1.383916 & -1.613779 \\
\hline 15 & 1 & 0 & 0.432281 & 3.867939 & -1.100256 \\
\hline 16 & 1 & 0 & 1.993048 & 3.079395 & -0.803091 \\
\hline 17 & 1 & 0 & 0.973456 & 3.583464 & 0.554792 \\
\hline 18 & 1 & 0 & 2.669023 & 1.278756 & -1.508980 \\
\hline 19 & 1 & 0 & 2.548955 & -0.596161 & 0.917589 \\
\hline 20 & 1 & 0 & 2.899572 & 1.137223 & 0.958448 \\
\hline 21 & 1 & 0 & 0.310900 & -2.897747 & -1.470520 \\
\hline 22 & 1 & 0 & -2.145361 & -3.200589 & -0.419025 \\
\hline 23 & 1 & 0 & -3.693553 & -1.206389 & -1.347434 \\
\hline 24 & 1 & 0 & -2.209421 & 0.310615 & -2.996729 \\
\hline 25 & 1 & 0 & 0.263465 & -0.730432 & -3.068830 \\
\hline 26 & 6 & 0 & 0.142395 & -2.158691 & 2.130305 \\
\hline 27 & 1 & 0 & -0.346425 & -2.088911 & 3.112337 \\
\hline 28 & 1 & 0 & 0.015179 & -3.190380 & 1.765076 \\
\hline 29 & 1 & 0 & 1.218354 & -1.993356 & 2.289731 \\
\hline 30 & 1 & 0 & 2.301256 & -0.440162 & -1.595577 \\
\hline 31 & 1 & 0 & -1.886314 & 4.257963 & -0.415933 \\
\hline 32 & 1 & 0 & -2.333403 & 3.201465 & 1.714021 \\
\hline 33 & 1 & 0 & -3.576507 & 2.521390 & -0.255695 \\
\hline 34 & 7 & 0 & 4.436128 & 0.021836 & 0.096639 \\
\hline 35 & 6 & 0 & 5.221048 & 0.269777 & 1.306633 \\
\hline 36 & 1 & 0 & 5.057026 & 1.296763 & 1.649202 \\
\hline 37 & 1 & 0 & 4.970680 & -0.417355 & 2.138612 \\
\hline 38 & 1 & 0 & 6.286277 & 0.152944 & 1.079494 \\
\hline 39 & 6 & 0 & 4.708705 & -1.324382 & -0.408794 \\
\hline 40 & 1 & 0 & 5.779781 & -1.425037 & -0.613778 \\
\hline 41 & 1 & 0 & 4.417223 & -2.118671 & 0.307017 \\
\hline 42 & 1 & 0 & 4.174943 & -1.498479 & -1.347270 \\
\hline 43 & 6 & 0 & -2.577016 & -0.517519 & 2.082614 \\
\hline 44 & 8 & 0 & -2.465224 & -0.866765 & 3.189768 \\
\hline 45 & 8 & 0 & -2.965905 & -0.090376 & 1.043695 \\
\hline
\end{tabular}

Complex II: $E=-941.264882207$

Input orientation:

\begin{tabular}{|c|c|c|c|c|c|}
\hline \multirow{2}{*}{$\begin{array}{l}\text { Center } \\
\text { Number }\end{array}$} & \multirow{2}{*}{$\begin{array}{l}\text { Atomic } \\
\text { Number }\end{array}$} & \multirow{2}{*}{$\begin{array}{c}\text { Atomic } \\
\text { Type }\end{array}$} & \multicolumn{3}{|c|}{ Coordinates (Angstroms) } \\
\hline & & & $\mathrm{X}$ & $\mathrm{Y}$ & $\mathrm{Z}$ \\
\hline------ & ----- & & --------- & -------- & -------- \\
\hline 1 & 22 & 0 & -1.122738 & -0.359755 & -0.226730 \\
\hline 2 & 7 & 0 & 0.769226 & 0.532156 & -0.621967 \\
\hline 3 & 7 & 0 & -1.061921 & 1.698602 & -0.287240 \\
\hline 4 & 7 & 0 & -0.728284 & -1.068770 & 1.501386 \\
\hline 5 & 6 & 0 & 0.269359 & 1.751804 & -0.499606 \\
\hline 6 & 6 & 0 & 1.044173 & 3.038078 & -0.575755 \\
\hline
\end{tabular}




\begin{tabular}{|c|c|c|c|c|c|}
\hline 7 & 6 & 0 & 2.157764 & 0.242279 & -0.944623 \\
\hline 8 & 6 & 0 & 3.060456 & 0.237535 & 0.300685 \\
\hline 9 & 14 & 0 & -2.183616 & 2.901009 & 0.343758 \\
\hline 10 & 6 & 0 & -1.798494 & -2.452048 & -1.094219 \\
\hline 11 & 6 & 0 & -0.568039 & -2.142239 & -1.720559 \\
\hline 12 & 6 & 0 & -0.763688 & -0.992256 & -2.527761 \\
\hline 13 & 6 & 0 & -2.110188 & -0.584556 & -2.393360 \\
\hline 14 & 6 & 0 & -2.749194 & -1.483280 & -1.500856 \\
\hline 15 & 1 & 0 & 0.506771 & 3.765552 & -1.190546 \\
\hline 16 & 1 & 0 & 2.045468 & 2.897140 & -0.986844 \\
\hline 17 & 1 & 0 & 1.147432 & 3.471214 & 0.426702 \\
\hline 18 & 1 & 0 & 2.566769 & 0.962021 & -1.666832 \\
\hline 19 & 1 & 0 & 2.674602 & -0.497001 & 1.033803 \\
\hline 20 & 1 & 0 & 2.991586 & 1.223472 & 0.776125 \\
\hline 21 & 1 & 0 & 0.365484 & -2.675792 & -1.585492 \\
\hline 22 & 1 & 0 & -1.973338 & -3.253910 & -0.388210 \\
\hline 23 & 1 & 0 & -3.767361 & -1.409239 & -1.142620 \\
\hline 24 & 1 & 0 & -2.562707 & 0.284695 & -2.853638 \\
\hline 25 & 1 & 0 & -0.004319 & -0.491211 & -3.113655 \\
\hline 26 & 6 & 0 & 0.145216 & -1.899892 & 2.309692 \\
\hline 27 & 1 & 0 & 0.496651 & -1.350643 & 3.191437 \\
\hline 28 & 1 & 0 & -0.378408 & -2.795134 & 2.666300 \\
\hline 29 & 1 & 0 & 1.013623 & -2.204104 & 1.719346 \\
\hline 30 & 1 & 0 & 2.179907 & -0.739970 & -1.429474 \\
\hline 31 & 1 & 0 & -1.804943 & 4.254951 & -0.194940 \\
\hline 32 & 1 & 0 & -2.151796 & 2.982926 & 1.838778 \\
\hline 33 & 1 & 0 & -3.553862 & 2.563645 & -0.136426 \\
\hline 34 & 7 & 0 & 4.465587 & 0.001267 & -0.042020 \\
\hline 35 & 6 & 0 & 5.350349 & 0.428675 & 1.043554 \\
\hline 36 & 1 & 0 & 5.195520 & 1.492947 & 1.248851 \\
\hline 37 & 1 & 0 & 5.191288 & -0.133404 & 1.983994 \\
\hline 38 & 1 & 0 & 6.392581 & 0.290008 & 0.737290 \\
\hline 39 & 6 & 0 & 4.721365 & -1.400650 & -0.376769 \\
\hline 40 & 1 & 0 & 5.773511 & -1.519821 & -0.655106 \\
\hline 41 & 1 & 0 & 4.506328 & -2.089620 & 0.463818 \\
\hline 42 & 1 & 0 & 4.117579 & -1.708076 & -1.235418 \\
\hline 43 & 6 & 0 & -1.986970 & -0.714822 & 1.993840 \\
\hline 44 & 8 & 0 & -2.421737 & -0.937157 & 3.105036 \\
\hline 45 & 8 & 0 & -2.633567 & -0.104681 & 0.986402 \\
\hline
\end{tabular}

Complex TS II-III : $\mathrm{E}=-941.232488906$

Input orientation:

\begin{tabular}{|c|c|c|c|c|c|}
\hline \multirow{2}{*}{$\begin{array}{l}\text { Center } \\
\text { Number }\end{array}$} & \multirow{2}{*}{$\begin{array}{l}\text { Atomic } \\
\text { Number }\end{array}$} & \multirow{2}{*}{$\begin{array}{l}\text { Atomic } \\
\text { Type }\end{array}$} & \multicolumn{3}{|c|}{ Coordinates (Angstroms) } \\
\hline & & & $\mathrm{X}$ & $\mathrm{Y}$ & Z \\
\hline------ & ----- & ------- & -------- & -------- & -------- \\
\hline 1 & 22 & 0 & -1.233687 & -0.375476 & 0.078243 \\
\hline 2 & 7 & 0 & 0.492982 & 0.731485 & -0.572097 \\
\hline 3 & 7 & 0 & -1.308167 & 1.755481 & 0.185976 \\
\hline 4 & 7 & 0 & 0.206054 & -2.039175 & 0.307963 \\
\hline 5 & 6 & 0 & -0.030495 & 1.892494 & -0.200062 \\
\hline 6 & 6 & 0 & 0.684635 & 3.219119 & -0.179267 \\
\hline 7 & 6 & 0 & 1.870676 & 0.594510 & -1.021486 \\
\hline 8 & 6 & 0 & 2.872024 & 0.523469 & 0.144646 \\
\hline 9 & 14 & 0 & -2.284180 & 2.908216 & 1.065805 \\
\hline 10 & 6 & 0 & -3.081700 & -1.769024 & -0.443074 \\
\hline 11 & 6 & 0 & -2.139570 & -2.039354 & -1.464160 \\
\hline 12 & 6 & 0 & -1.998589 & -0.876463 & -2.265505 \\
\hline 13 & 6 & 0 & -2.838564 & 0.117179 & -1.725878 \\
\hline 14 & 6 & 0 & -3.502828 & -0.428373 & -0.593709 \\
\hline
\end{tabular}




$\begin{array}{rrrrrr}15 & 1 & 0 & 0.051558 & 3.990750 & -0.629115 \\ 16 & 1 & 0 & 1.637696 & 3.197730 & -0.710639 \\ 17 & 1 & 0 & 0.881485 & 3.519074 & 0.857570 \\ 18 & 1 & 0 & 2.166756 & 1.426397 & -1.676240 \\ 19 & 1 & 0 & 2.680230 & -0.389875 & 0.743818 \\ 20 & 1 & 0 & 2.683183 & 1.376145 & 0.807808 \\ 21 & 1 & 0 & -1.623722 & -2.980329 & -1.609798 \\ 22 & 1 & 0 & -3.386713 & -2.447918 & 0.342859 \\ 23 & 1 & 0 & -4.195470 & 0.095753 & 0.052794 \\ 24 & 1 & 0 & -2.923278 & 1.137302 & -2.076163 \\ 25 & 1 & 0 & -1.337859 & -0.760577 & -3.116155 \\ 26 & 6 & 0 & 1.094953 & -2.877743 & -0.517501 \\ 27 & 1 & 0 & 2.142778 & -2.600327 & -0.366932 \\ 28 & 1 & 0 & 0.971741 & -3.941049 & -0.287619 \\ 29 & 1 & 0 & 0.834279 & -2.711392 & -1.563258 \\ 30 & 1 & 0 & 1.926187 & -0.314436 & -1.629252 \\ 31 & 1 & 0 & -2.425546 & 4.203858 & 0.310324 \\ 32 & 1 & 0 & -1.719725 & 3.249075 & 2.417198 \\ 33 & 1 & 0 & -3.643635 & 2.314199 & 1.240208 \\ 34 & 7 & 0 & 4.265591 & 0.612045 & -0.301440 \\ 35 & 6 & 0 & 5.157113 & 0.859820 & 0.832839 \\ 36 & 1 & 0 & 4.863825 & 1.786713 & 1.336462 \\ 37 & 1 & 0 & 5.148554 & 0.042556 & 1.580006 \\ 38 & 1 & 0 & 6.183548 & 0.979386 & 0.469987 \\ 39 & 6 & 0 & 4.692694 & -0.583527 & -1.026389 \\ 40 & 1 & 0 & 5.733654 & -0.462001 & -1.343500 \\ 41 & 1 & 0 & 0.628026 & -1.506151 & -0.413731 \\ 42 & 1 & 0.088022 & -0.723005 & -1.927142 \\ 43 & -104070 & -2.248727 & 1.541769 \\ 44 & 1 & 0 & -1.348944 & -0.660757 & 1.712564 \\ 45 & 1 & 0 & ------------------------\end{array}$

Complex III: $\mathrm{E}=-941.233682766$

Z-Matrix orientation:

\begin{tabular}{|c|c|c|c|c|c|}
\hline \multirow{2}{*}{$\begin{array}{l}\text { Center } \\
\text { Number }\end{array}$} & \multirow{2}{*}{$\begin{array}{l}\text { Atomic } \\
\text { Number }\end{array}$} & \multirow{2}{*}{$\begin{array}{l}\text { Atomic } \\
\text { Type }\end{array}$} & \multicolumn{3}{|c|}{ Coordinates (Angstroms) } \\
\hline & & & $\mathrm{X}$ & $\mathrm{Y}$ & Z \\
\hline 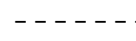 & --- & & ------- & ------- & -------- \\
\hline 1 & 22 & 0 & -0.333858 & 0.160436 & -1.255463 \\
\hline 2 & 7 & 0 & 0.691169 & -0.604984 & 0.490286 \\
\hline 3 & 7 & 0 & 1.797464 & 0.072645 & -1.302077 \\
\hline 4 & 7 & 0 & -2.100883 & 0.315725 & 0.265006 \\
\hline 5 & 6 & 0 & 1.883770 & -0.331263 & -0.026091 \\
\hline 6 & 6 & 0 & 3.203529 & -0.425907 & 0.697759 \\
\hline 7 & 6 & 0 & 0.521645 & -1.039695 & 1.869584 \\
\hline 8 & 6 & 0 & 0.506651 & 0.133758 & 2.864455 \\
\hline 9 & 14 & 0 & 3.042934 & 0.857292 & -2.244254 \\
\hline 10 & 6 & 0 & -1.706866 & -0.146949 & -3.183365 \\
\hline 11 & 6 & 0 & -2.131617 & -1.157582 & -2.290572 \\
\hline 12 & 6 & 0 & -1.076113 & -2.097477 & -2.137753 \\
\hline 13 & 6 & 0 & 0.006256 & -1.651013 & -2.917932 \\
\hline 14 & 6 & 0 & -0.375126 & -0.437885 & -3.555097 \\
\hline 15 & 1 & 0 & 3.934178 & -0.948529 & 0.071791 \\
\hline 16 & 1 & 0 & 3.132360 & -0.945219 & 1.654761 \\
\hline 17 & 1 & 0 & 3.594697 & 0.581391 & 0.887758 \\
\hline 18 & 1 & 0 & 1.313991 & -1.737234 & 2.175346 \\
\hline 19 & 1 & 0 & -0.369142 & 0.782625 & 2.659399 \\
\hline 20 & 1 & 0 & 1.398025 & 0.745820 & 2.682188 \\
\hline 21 & 1 & 0 & -3.108893 & -1.214262 & -1.827364 \\
\hline 22 & 1 & 0 & -2.276639 & 0.722777 & -3.483905 \\
\hline
\end{tabular}




\begin{tabular}{|c|c|c|c|c|c|}
\hline 23 & 1 & 0 & 0.247772 & 0.164325 & -4.204305 \\
\hline 24 & 1 & 0 & 0.981837 & -2.115418 & -2.977582 \\
\hline 25 & 1 & 0 & -1.088550 & -2.982611 & -1.512683 \\
\hline 26 & 6 & 0 & -2.886588 & -0.731194 & 0.947531 \\
\hline 27 & 1 & 0 & -2.773418 & -0.658660 & 2.032946 \\
\hline 28 & 1 & 0 & -3.947605 & -0.658114 & 0.689967 \\
\hline 29 & 1 & 0 & -2.510079 & -1.697400 & 0.613842 \\
\hline 30 & 1 & 0 & -0.418056 & -1.597891 & 1.926158 \\
\hline 31 & 1 & 0 & 4.250048 & -0.028403 & -2.413731 \\
\hline 32 & 1 & 0 & 3.527765 & 2.139998 & -1.626296 \\
\hline 33 & 1 & 0 & 2.482111 & 1.149096 & -3.597233 \\
\hline 34 & 7 & 0 & 0.550225 & -0.307117 & 4.261902 \\
\hline 35 & 6 & 0 & 0.845332 & 0.818941 & 5.149508 \\
\hline 36 & 1 & 0 & 1.802668 & 1.268986 & 4.867358 \\
\hline 37 & 1 & 0 & 0.070421 & 1.609580 & 5.122314 \\
\hline 38 & 1 & 0 & 0.928888 & 0.458367 & 6.180295 \\
\hline 39 & 6 & 0 & -0.689959 & -0.962057 & 4.674039 \\
\hline 40 & 1 & 0 & -0.607012 & -1.268628 & 5.721908 \\
\hline 41 & 1 & 0 & -1.577887 & -0.302963 & 4.580631 \\
\hline 42 & 1 & 0 & -0.863669 & -1.864299 & 4.080347 \\
\hline 43 & 6 & 0 & -2.340785 & 1.522341 & 0.400912 \\
\hline 44 & 8 & 0 & -2.591282 & 2.649198 & 0.550019 \\
\hline 45 & 8 & 0 & -0.476649 & 1.788221 & -1.316041 \\
\hline
\end{tabular}

Complex TS III-IV $: E=-941.230487682$

Input orientation:

\begin{tabular}{|c|c|c|c|c|c|}
\hline \multirow{2}{*}{$\begin{array}{l}\text { Center } \\
\text { Number }\end{array}$} & \multirow{2}{*}{$\begin{array}{l}\text { Atomic } \\
\text { Number }\end{array}$} & \multirow{2}{*}{$\begin{array}{l}\text { Atomic } \\
\text { Type }\end{array}$} & \multicolumn{3}{|c|}{ Coordinates (Angstroms) } \\
\hline & & & $\mathrm{X}$ & $\mathrm{Y}$ & Z \\
\hline-- & & ---- & -------- & -------- & -------- \\
\hline 1 & 22 & 0 & -0.221749 & 0.225826 & -1.347971 \\
\hline 2 & 7 & 0 & 0.788911 & -0.524919 & 0.344403 \\
\hline 3 & 7 & 0 & 1.869600 & 0.300513 & -1.421729 \\
\hline 4 & 7 & 0 & -2.535942 & 0.009057 & 0.741911 \\
\hline 5 & 6 & 0 & 1.966692 & -0.188433 & -0.168569 \\
\hline 6 & 6 & 0 & 3.293700 & -0.311852 & 0.534741 \\
\hline 7 & 6 & 0 & 0.619600 & -1.005684 & 1.708621 \\
\hline 8 & 6 & 0 & 0.654660 & 0.134036 & 2.740334 \\
\hline 9 & 14 & 0 & 3.128494 & 1.056055 & -2.371361 \\
\hline 10 & 6 & 0 & -1.455549 & -0.019651 & -3.364376 \\
\hline 11 & 6 & 0 & -2.086625 & -0.818495 & -2.384358 \\
\hline 12 & 6 & 0 & -1.226140 & -1.915617 & -2.085685 \\
\hline 13 & 6 & 0 & -0.063110 & -1.779902 & -2.865381 \\
\hline 14 & 6 & 0 & -0.190608 & -0.592934 & -3.635389 \\
\hline 15 & 1 & 0 & 4.024713 & -0.783045 & -0.130935 \\
\hline 16 & 1 & 0 & 3.233685 & -0.897364 & 1.453402 \\
\hline 17 & 1 & 0 & 3.673922 & 0.684911 & 0.789443 \\
\hline 18 & 1 & 0 & 1.388733 & -1.743547 & 1.976273 \\
\hline 19 & 1 & 0 & -0.155242 & 0.855602 & 2.522226 \\
\hline 20 & 1 & 0 & 1.599524 & 0.677596 & 2.619202 \\
\hline 21 & 1 & 0 & -3.057079 & -0.625116 & -1.945846 \\
\hline 22 & 1 & 0 & -1.842000 & 0.904079 & -3.775118 \\
\hline 23 & 1 & 0 & 0.552859 & -0.194838 & -4.315385 \\
\hline 24 & 1 & 0 & 0.807804 & -2.422755 & -2.832729 \\
\hline 25 & 1 & 0 & -1.420588 & -2.705866 & -1.369754 \\
\hline 26 & 6 & 0 & -3.380186 & -1.142220 & 1.039119 \\
\hline 27 & 1 & 0 & -3.410791 & -1.343959 & 2.114986 \\
\hline 28 & 1 & 0 & -4.403653 & -0.991133 & 0.680546 \\
\hline 29 & 1 & 0 & -2.964992 & -2.016053 & 0.535608 \\
\hline 30 & 1 & 0 & -0.344018 & -1.521390 & 1.746396 \\
\hline
\end{tabular}




\begin{tabular}{|c|c|c|c|c|c|}
\hline 31 & 1 & 0 & 4.245184 & 0.096212 & -2.685490 \\
\hline 32 & 1 & 0 & 3.745535 & 2.237158 & -1.675618 \\
\hline 33 & 1 & 0 & 2.511838 & 1.507596 & -3.654636 \\
\hline 34 & 7 & 0 & 0.605843 & -0.362957 & 4.120060 \\
\hline 35 & 6 & 0 & 1.026482 & 0.676806 & 5.061355 \\
\hline 36 & 1 & 0 & 2.044678 & 1.000678 & 4.821830 \\
\hline 37 & 1 & 0 & 0.368491 & 1.566943 & 5.050915 \\
\hline 38 & 1 & 0 & 1.030164 & 0.267369 & 6.077286 \\
\hline 39 & 6 & 0 & -0.722981 & -0.857307 & 4.480791 \\
\hline 40 & 1 & 0 & -0.701116 & -1.235955 & 5.508122 \\
\hline 41 & 1 & 0 & -1.505329 & -0.074750 & 4.418730 \\
\hline 42 & 1 & 0 & -1.011557 & -1.687315 & 3.829413 \\
\hline 43 & 6 & 0 & -2.662698 & 1.177050 & 1.068614 \\
\hline 44 & 8 & 0 & -2.694112 & 2.314923 & 1.346916 \\
\hline 45 & 8 & 0 & -0.689576 & 1.760746 & -1.118900 \\
\hline
\end{tabular}

Complex IV: $\mathrm{E}=-733.342611235$

Input orientation:

\begin{tabular}{|c|c|c|c|c|c|}
\hline \multirow{2}{*}{$\begin{array}{l}\text { Center } \\
\text { Number }\end{array}$} & \multirow{2}{*}{$\begin{array}{l}\text { Atomic } \\
\text { Number }\end{array}$} & \multirow{2}{*}{$\begin{array}{l}\text { Atomic } \\
\text { Type }\end{array}$} & \multicolumn{3}{|c|}{ Coordinates (Angstroms) } \\
\hline & & & $\mathrm{X}$ & $\mathrm{Y}$ & Z \\
\hline--- & ----- & ----- & -------1 & --------- & --------- \\
\hline 1 & 22 & 0 & -.597525 & .412507 & -1.125326 \\
\hline 2 & 7 & 0 & .378536 & -.492528 & .476127 \\
\hline 3 & 7 & 0 & 1.460621 & .200360 & -1.351759 \\
\hline 4 & 8 & 0 & -.937548 & 1.938845 & -.695382 \\
\hline 5 & 6 & 0 & 1.558996 & -.296701 & -.094819 \\
\hline 6 & 6 & 0 & 2.895542 & -.562994 & .545524 \\
\hline 7 & 6 & 0 & .173081 & -.895521 & 1.858454 \\
\hline 8 & 6 & 0 & .084903 & .320055 & 2.796117 \\
\hline 9 & 14 & 0 & 2.757903 & .695216 & -2.415346 \\
\hline 10 & 6 & 0 & -2.624263 & .154207 & -2.304285 \\
\hline 11 & 6 & 0 & -2.603270 & -.866530 & -1.324579 \\
\hline 12 & 6 & 0 & -1.590542 & -1.803865 & -1.678690 \\
\hline 13 & 6 & 0 & -.964504 & -1.343563 & -2.851343 \\
\hline 14 & 6 & 0 & -1.585269 & -.117936 & -3.226958 \\
\hline 15 & 1 & 0 & 3.517998 & -1.162419 & -.127777 \\
\hline 16 & 1 & 0 & 2.814152 & -1.087299 & 1.498283 \\
\hline 17 & 1 & 0 & 3.416378 & .385921 & .720328 \\
\hline 18 & 1 & 0 & .968982 & -1.561329 & 2.214710 \\
\hline 19 & 1 & 0 & -.689288 & 1.018071 & 2.424137 \\
\hline 20 & 1 & 0 & 1.041033 & .855804 & 2.748037 \\
\hline 21 & 1 & 0 & -3.258461 & -.929201 & -.462609 \\
\hline 22 & 1 & 0 & -3.264540 & 1.026414 & -2.303897 \\
\hline 23 & 1 & 0 & -1.320286 & .495904 & -4.080895 \\
\hline 24 & 1 & 0 & -.124869 & -1.809092 & -3.352153 \\
\hline 25 & 1 & 0 & -1.319208 & -2.687882 & -1.114947 \\
\hline 26 & 1 & 0 & -.758673 & -1.470171 & 1.893668 \\
\hline 27 & 1 & 0 & 3.652755 & -.458604 & -2.778858 \\
\hline 28 & 1 & 0 & 3.628794 & 1.760544 & -1.810236 \\
\hline 29 & 1 & 0 & 2.121940 & 1.224766 & -3.659544 \\
\hline 30 & 7 & 0 & -.141121 & -.081670 & 4.188800 \\
\hline 31 & 6 & 0 & .248530 & .985395 & 5.112753 \\
\hline 32 & 1 & 0 & 1.306142 & 1.229993 & 4.969389 \\
\hline 33 & 1 & 0 & -.342720 & 1.912369 & 4.984128 \\
\hline 34 & 1 & 0 & .116667 & .638483 & 6.143328 \\
\hline 35 & 6 & 0 & -1.534443 & -.464839 & 4.425688 \\
\hline 36 & 1 & 0 & -1.647194 & -.797284 & 5.462975 \\
\hline 37 & 1 & 0 & -2.244472 & .366590 & 4.248789 \\
\hline 38 & 1 & 0 & -1.818635 & -1.299904 & 3.778882 \\
\hline
\end{tabular}


$\underline{\mathrm{CO}_{2}} \underline{2}$ attack from $\mathrm{CH}_{2} \underline{\mathrm{CH}}_{2} \underline{\mathrm{NMe}}_{2} \underline{\text { side }}$

TS I-II $: E=-941.222939456$

Input orientation:

\begin{tabular}{|c|c|c|c|c|c|}
\hline \multirow{2}{*}{$\begin{array}{l}\text { Center } \\
\text { Number }\end{array}$} & \multirow{2}{*}{$\begin{array}{l}\text { Atomic } \\
\text { Number }\end{array}$} & \multirow{2}{*}{$\begin{array}{l}\text { Atomic } \\
\text { Type }\end{array}$} & \multicolumn{3}{|c|}{ Coordinates (Angstroms) } \\
\hline & & & $\mathrm{X}$ & $\mathrm{Y}$ & $\mathrm{Z}$ \\
\hline- & & & ----- & ---------- & --------- \\
\hline 1 & 22 & 0 & -0.279538 & -0.326255 & -1.142006 \\
\hline 2 & 7 & 0 & 0.602839 & -0.453992 & 0.806667 \\
\hline 3 & 7 & 0 & 1.831074 & -0.145198 & -1.009328 \\
\hline 4 & 7 & 0 & -0.654728 & 1.254542 & -1.660118 \\
\hline 5 & 6 & 0 & 1.822466 & -0.246735 & 0.332651 \\
\hline 6 & 6 & 0 & 3.078984 & -0.118820 & 1.155970 \\
\hline 7 & 6 & 0 & 0.306418 & -0.635436 & 2.219961 \\
\hline 8 & 6 & 0 & 0.164961 & 0.702446 & 2.964643 \\
\hline 9 & 14 & 0 & 3.110830 & 0.439501 & -2.036999 \\
\hline 10 & 6 & 0 & -1.760981 & -1.417531 & -2.622307 \\
\hline 11 & 6 & 0 & -1.646414 & -2.287885 & -1.510001 \\
\hline 12 & 6 & 0 & -0.316062 & -2.767143 & -1.454346 \\
\hline 13 & 6 & 0 & 0.391946 & -2.192038 & -2.535447 \\
\hline 14 & 6 & 0 & -0.495056 & -1.357730 & -3.258660 \\
\hline 15 & 1 & 0 & 3.877991 & -0.726182 & 0.718455 \\
\hline 16 & 1 & 0 & 2.937540 & -0.426429 & 2.193651 \\
\hline 17 & 1 & 0 & 3.419917 & 0.924122 & 1.155714 \\
\hline 18 & 1 & 0 & 1.076244 & -1.234378 & 2.726167 \\
\hline 19 & 1 & 0 & -0.635785 & 1.305219 & 2.491447 \\
\hline 20 & 1 & 0 & 1.097769 & 1.265870 & 2.838372 \\
\hline 21 & 1 & 0 & -2.433911 & -2.516553 & -0.802017 \\
\hline 22 & 1 & 0 & -2.652999 & -0.886550 & -2.930942 \\
\hline 23 & 1 & 0 & -0.248248 & -0.766909 & -4.131832 \\
\hline 24 & 1 & 0 & 1.441839 & -2.337416 & -2.751886 \\
\hline 25 & 1 & 0 & 0.094451 & -3.426826 & -0.700216 \\
\hline 26 & 6 & 0 & -0.917261 & 2.415592 & -2.461871 \\
\hline 27 & 1 & 0 & -1.361808 & 2.147278 & -3.433070 \\
\hline 28 & 1 & 0 & -1.607168 & 3.108266 & -1.959315 \\
\hline 29 & 1 & 0 & 0.014150 & 2.965061 & -2.661191 \\
\hline 30 & 1 & 0 & -0.627635 & -1.202308 & 2.282761 \\
\hline 31 & 1 & 0 & 4.377466 & -0.362374 & -1.886633 \\
\hline 32 & 1 & 0 & 3.475962 & 1.875818 & -1.772051 \\
\hline 33 & 1 & 0 & 2.643016 & 0.316147 & -3.451979 \\
\hline 34 & 7 & 0 & -0.055744 & 0.521482 & 4.402679 \\
\hline 35 & 6 & 0 & 0.210933 & 1.761762 & 5.132313 \\
\hline 36 & 1 & 0 & 1.242859 & 2.081357 & 4.952846 \\
\hline 37 & 1 & 0 & -0.464035 & 2.591435 & 4.844592 \\
\hline 38 & 1 & 0 & 0.091934 & 1.584454 & 6.206682 \\
\hline 39 & 6 & 0 & -1.409304 & 0.047850 & 4.695414 \\
\hline 40 & 1 & 0 & -1.514617 & -0.100995 & 5.775191 \\
\hline 41 & 1 & 0 & -2.193852 & 0.758130 & 4.366533 \\
\hline 42 & 1 & 0 & -1.593073 & -0.913636 & 4.208516 \\
\hline 43 & 6 & 0 & -2.495626 & 1.079803 & 0.007115 \\
\hline 44 & 8 & 0 & -2.969822 & 2.136097 & -0.132338 \\
\hline 45 & 8 & 0 & -2.221914 & -0.028678 & 0.331142 \\
\hline
\end{tabular}

Complex II: $\mathrm{E}=-941.266708942$

$\mathrm{Ti},-0.9770359503,-0.339341728,-0.2570775831$

$\mathrm{N}, 0.8972928839,0.6617931527,-0.6967110892$

$\mathrm{N},-0.9327393331,1.6781056382,0.0129741615$ 
$\mathrm{N},-2.0831299987,-0.8683852935,1.2520171115$

C, $0.4043185463,1.7896856002,-0.2490998264$

C, $1.154943923,3.0703209452,-0.010184949$

C, $2.3044474521,0.4227012924,-0.9772727203$

C, $3.0949594973,0.0269467362,0.2821271643$

$\mathrm{Si},-1.9936827085,2.8197291628,0.8124231483$

C, $-2.3240162467,-1.8762075356,-1.4269783876$

C, $-1.0322217837,-1.8967407623,-2.0035903148$

C, $-0.8141961352,-0.6342168972,-2.6205250122$

C, $-1.9656559216,0.1587822604,-2.4200650935$

C, $-2.895475992,-0.6013790489,-1.6739110222$

$\mathrm{H}, 0.6268853694,3.9101885718,-0.4738790323$

$\mathrm{H}, 2.1714079918,3.0364734281,-0.4053411795$

$\mathrm{H}, 1.2151746852,3.2712405008,1.0664274284$

$\mathrm{H}, 2.7823043691,1.3016435651,-1.4312646776$

$\mathrm{H}, 2.6154691883,-0.842566297,0.7633737172$

$\mathrm{H}, 3.0352066271,0.857451926,0.9965660716$

$\mathrm{H},-0.3289828401,-2.7199468692,-1.9603725415$

$\mathrm{H},-2.7762794668,-2.677606109,-0.8568363413$

$\mathrm{H},-3.8631360896,-0.2625475927,-1.3250853768$

$\mathrm{H},-2.0905539657,1.1870623341,-2.7329446354$

$\mathrm{H}, 0.0879504299,-0.3250478641,-3.1307760745$

C, $-3.4302243955,-1.0910877545,1.7451037654$

$\mathrm{H},-4.0544058214,-1.5764093405,0.9832982526$

$\mathrm{H},-3.390965518,-1.7309907287,2.632250829$

$\mathrm{H},-3.909435849,-0.1435921338,2.0173005263$

$\mathrm{H}, 2.3542470943,-0.3825832475,-1.7185976121$

$\mathrm{H},-1.9598969158,4.1617403084,0.1342477839$

$\mathrm{H},-1.6380383366,3.0327361493,2.2558243242$

$\mathrm{H},-3.38007694,2.270205734,0.7192207017$

$\mathrm{N}, 4.5124839161,-0.2005118664,-0.0295777401$

C, $5.3401014278,-0.0726403577,1.1718334264$

$\mathrm{H}, 5.2197132213,0.9288140872,1.5983217464$

$\mathrm{H}, 5.0922513875,-0.814825372,1.9545099639$

$\mathrm{H}, 6.3938544992,-0.2021453196,0.9016124411$

C, $4.7272846031,-1.5115322608,-0.6463991126$

$\mathrm{H}, 5.7864910684,-1.6223778853,-0.901988464$

$\mathrm{H}, 4.439631487,-2.3504426909,0.0163994993$

$\mathrm{H}, 4.152929109,-1.597440894,-1.5736857359$

C, $-1.0017380669,-1.4281320346,1.9113546438$

$0,-0.979466606,-2.0549772845,2.9483147244$

$0,0.0659778956,-1.164489845,1.1126763587$

TS $_{\text {II-III }}: E=-941.234247961$

Input orientation:

\begin{tabular}{|c|c|c|c|c|c|}
\hline \multirow{2}{*}{$\begin{array}{l}\text { Center } \\
\text { Number }\end{array}$} & \multirow{2}{*}{$\begin{array}{l}\text { Atomic } \\
\text { Number }\end{array}$} & \multirow{2}{*}{$\begin{array}{c}\text { Atomic } \\
\text { Type }\end{array}$} & \multicolumn{3}{|c|}{ Coordinates (Angstroms) } \\
\hline & & & $\mathrm{X}$ & $\mathrm{Y}$ & Z \\
\hline- & 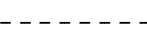 & - & -------- & -------- & ------- \\
\hline 1 & 22 & 0 & -0.455600 & -0.208085 & -0.786588 \\
\hline 2 & 7 & 0 & 0.759956 & -0.679408 & 0.879570 \\
\hline 3 & 7 & 0 & 1.664056 & 0.198117 & -0.924973 \\
\hline 4 & 7 & 0 & -0.676241 & 1.378032 & -2.326549 \\
\hline 5 & 6 & 0 & 1.853362 & -0.154688 & 0.368966 \\
\hline 6 & 6 & 0 & 3.153197 & 0.047988 & 1.102748 \\
\hline 7 & 6 & 0 & 0.560360 & -1.029375 & 2.275442 \\
\hline 8 & 6 & 0 & 0.081197 & 0.170425 & 3.110193 \\
\hline 9 & 14 & 0 & 2.824212 & 0.886246 & -2.024140 \\
\hline 10 & 6 & 0 & -2.281212 & -1.428017 & -1.665479 \\
\hline 11 & 6 & 0 & -1.839417 & -2.106377 & -0.507606 \\
\hline 12 & 6 & 0 & -0.560037 & -2.657391 & -0.785468 \\
\hline
\end{tabular}




\begin{tabular}{|c|c|c|c|c|c|}
\hline 13 & 6 & 0 & -0.212131 & -2.324546 & -2.112457 \\
\hline 14 & 6 & 0 & -1.270200 & -1.554735 & -2.651874 \\
\hline 15 & 1 & 0 & 3.966769 & -0.464469 & 0.578709 \\
\hline 16 & 1 & 0 & 3.114982 & -0.323631 & 2.127716 \\
\hline 17 & 1 & 0 & 3.405188 & 1.114105 & 1.134158 \\
\hline 18 & 1 & 0 & 1.473439 & -1.436922 & 2.729630 \\
\hline 19 & 1 & 0 & -0.805753 & 0.619763 & 2.629101 \\
\hline 20 & 1 & 0 & 0.871803 & 0.931363 & 3.095863 \\
\hline 21 & 1 & 0 & -2.372721 & -2.165940 & 0.433163 \\
\hline 22 & 1 & 0 & -3.207888 & -0.877175 & -1.764582 \\
\hline 23 & 1 & 0 & -1.306607 & -1.133868 & -3.649123 \\
\hline 24 & 1 & 0 & 0.718383 & -2.570544 & -2.608480 \\
\hline 25 & 1 & 0 & 0.056503 & -3.208702 & -0.087513 \\
\hline 26 & 6 & 0 & -0.291497 & 1.688052 & -3.719583 \\
\hline 27 & 1 & 0 & 0.101130 & 0.776522 & -4.169048 \\
\hline 28 & 1 & 0 & -1.152141 & 2.031890 & -4.302353 \\
\hline 29 & 1 & 0 & 0.489518 & 2.453715 & -3.746536 \\
\hline 30 & 1 & 0 & -0.188501 & -1.828982 & 2.304239 \\
\hline 31 & 1 & 0 & 4.236481 & 0.486557 & -1.698491 \\
\hline 32 & 1 & 0 & 2.787036 & 2.391254 & -2.060675 \\
\hline 33 & 1 & 0 & 2.521849 & 0.401170 & -3.409903 \\
\hline 34 & 7 & 0 & -0.158373 & -0.205898 & 4.510188 \\
\hline 35 & 6 & 0 & -0.118723 & 0.968193 & 5.383467 \\
\hline 36 & 1 & 0 & 0.858240 & 1.456260 & 5.301559 \\
\hline 37 & 1 & 0 & -0.900664 & 1.715865 & 5.147657 \\
\hline 38 & 1 & 0 & -0.254418 & 0.651739 & 6.423594 \\
\hline 39 & 6 & 0 & -1.434748 & -0.905139 & 4.671902 \\
\hline 40 & 1 & 0 & -1.552438 & -1.208271 & 5.717881 \\
\hline 41 & 1 & 0 & -2.305096 & -0.281016 & 4.390161 \\
\hline 42 & 1 & 0 & -1.454267 & -1.811996 & 4.060555 \\
\hline 43 & 6 & 0 & -1.374802 & 2.200741 & -1.683628 \\
\hline 44 & 8 & 0 & -2.014863 & 3.121450 & -1.355092 \\
\hline 45 & 8 & 0 & -1.401946 & 0.894183 & 0.027372 \\
\hline
\end{tabular}

Complex III: $E=-941.235832621$

Input orientation:

\begin{tabular}{|c|c|c|c|c|c|}
\hline \multirow{2}{*}{$\begin{array}{l}\text { Center } \\
\text { Number }\end{array}$} & \multirow{2}{*}{$\begin{array}{l}\text { Atomic } \\
\text { Number }\end{array}$} & \multirow{2}{*}{$\begin{array}{l}\text { Atomic } \\
\text { Type }\end{array}$} & \multicolumn{3}{|c|}{ Coordinates (Angstroms) } \\
\hline & & & $\mathrm{X}$ & $\mathrm{Y}$ & Z \\
\hline--- & ----- & & -------- & -------- & --------- \\
\hline 1 & 22 & 0 & -0.533070 & -0.103948 & -0.722855 \\
\hline 2 & 7 & 0 & 0.521348 & -0.830323 & 0.970096 \\
\hline 3 & 7 & 0 & 1.602278 & -0.346289 & -0.892150 \\
\hline 4 & 7 & 0 & -0.183311 & 1.162936 & -2.700115 \\
\hline 5 & 6 & 0 & 1.705322 & -0.645148 & 0.426811 \\
\hline 6 & 6 & 0 & 3.023527 & -0.729852 & 1.151933 \\
\hline 7 & 6 & 0 & 0.277215 & -1.002403 & 2.392632 \\
\hline 8 & 6 & 0 & 0.160915 & 0.346593 & 3.121780 \\
\hline 9 & 14 & 0 & 2.899730 & 0.082876 & -1.971301 \\
\hline 10 & 6 & 0 & -2.824739 & -0.743379 & -1.002959 \\
\hline 11 & 6 & 0 & -2.255310 & -1.633629 & -0.066801 \\
\hline 12 & 6 & 0 & -1.327478 & -2.468825 & -0.752441 \\
\hline 13 & 6 & 0 & -1.297047 & -2.071452 & -2.098725 \\
\hline 14 & 6 & 0 & -2.210360 & -0.987831 & -2.250671 \\
\hline 15 & 1 & 0 & 3.681046 & -1.451701 & 0.656713 \\
\hline 16 & 1 & 0 & 2.907386 & -1.026782 & 2.195039 \\
\hline 17 & 1 & 0 & 3.529085 & 0.242595 & 1.129015 \\
\hline 18 & 1 & 0 & 1.063116 & -1.600219 & 2.872746 \\
\hline 19 & 1 & 0 & -0.594365 & 0.973272 & 2.614582 \\
\hline 20 & 1 & 0 & 1.121467 & 0.867918 & 3.023869 \\
\hline
\end{tabular}




\begin{tabular}{|c|c|c|c|c|c|}
\hline 21 & 1 & 0 & -2.494167 & -1.677510 & 0.988523 \\
\hline 22 & 1 & 0 & -3.544774 & 0.034910 & -0.786894 \\
\hline 23 & 1 & 0 & -2.431485 & -0.463558 & -3.171686 \\
\hline 24 & 1 & 0 & -0.668986 & -2.495973 & -2.873311 \\
\hline 25 & 1 & 0 & -0.707362 & -3.231106 & -0.297903 \\
\hline 26 & 6 & 0 & -0.071739 & 0.746269 & -4.114193 \\
\hline 27 & 1 & 0 & 0.129665 & -0.323211 & -4.130792 \\
\hline 28 & 1 & 0 & -1.007965 & 0.942177 & -4.645381 \\
\hline 29 & 1 & 0 & 0.747559 & 1.266311 & -4.617227 \\
\hline 30 & 1 & 0 & -0.653975 & -1.569606 & 2.496971 \\
\hline 31 & 1 & 0 & 4.186497 & -0.616050 & -1.630645 \\
\hline 32 & 1 & 0 & 3.194784 & 1.559623 & -1.998611 \\
\hline 33 & 1 & 0 & 2.530318 & -0.321855 & -3.365649 \\
\hline 34 & 7 & 0 & -0.113214 & 0.170323 & 4.554591 \\
\hline 35 & 6 & 0 & 0.258387 & 1.367926 & 5.309805 \\
\hline 36 & 1 & 0 & 1.324016 & 1.577339 & 5.168974 \\
\hline 37 & 1 & 0 & -0.313870 & 2.267929 & 5.012074 \\
\hline 38 & 1 & 0 & 0.084659 & 1.192539 & 6.377254 \\
\hline 39 & 6 & 0 & -1.518690 & -0.157524 & 4.800735 \\
\hline 40 & 1 & 0 & -1.669735 & -0.325955 & 5.872501 \\
\hline 41 & 1 & 0 & -2.210152 & 0.643446 & 4.473602 \\
\hline 42 & 1 & 0 & -1.794035 & -1.078996 & 4.279592 \\
\hline 43 & 6 & 0 & -0.247209 & 2.350398 & -2.36043 \\
\hline 44 & 8 & 0 & -0.288993 & 3.470998 & -2.048977 \\
\hline 45 & 8 & 0 & -0.973993 & 1.331213 & -0.077793 \\
\hline
\end{tabular}

TS III-IV $: E=-941.232820501$

Input orientation:

\begin{tabular}{|c|c|c|c|c|c|}
\hline \multirow{2}{*}{$\begin{array}{l}\text { Center } \\
\text { Number }\end{array}$} & \multirow{2}{*}{$\begin{array}{l}\text { Atomic } \\
\text { Number }\end{array}$} & \multirow{2}{*}{$\begin{array}{c}\text { Atomic } \\
\text { Type }\end{array}$} & \multicolumn{3}{|c|}{ Coordinates (Angstroms) } \\
\hline & & & $\mathrm{X}$ & $\mathrm{Y}$ & Z \\
\hline & & & -------- & -------- & -------- \\
\hline 1 & 22 & 0 & -0.736205 & -0.157821 & -0.562984 \\
\hline 2 & 7 & 0 & 0.157666 & -0.861507 & 1.179813 \\
\hline 3 & 7 & 0 & 1.226210 & -0.870895 & -0.773436 \\
\hline 4 & 7 & 0 & 0.533640 & 1.429958 & -3.132920 \\
\hline 5 & 6 & 0 & 1.317457 & -1.045463 & 0.567052 \\
\hline 6 & 6 & 0 & 2.616943 & -1.379827 & 1.249523 \\
\hline 7 & 6 & 0 & -0.019180 & -0.779210 & 2.621769 \\
\hline 8 & 6 & 0 & 0.273279 & 0.631302 & 3.159684 \\
\hline 9 & 14 & 0 & 2.435110 & -1.160346 & -2.000214 \\
\hline 10 & 6 & 0 & -3.028632 & -0.725508 & -0.539081 \\
\hline 11 & 6 & 0 & -2.376578 & -1.964205 & -0.288969 \\
\hline 12 & 6 & 0 & -1.657654 & -2.327168 & -1.448641 \\
\hline 13 & 6 & 0 & -1.840625 & -1.302712 & -2.408452 \\
\hline 14 & 6 & 0 & -2.703689 & -0.320633 & -1.854615 \\
\hline 15 & 1 & 0 & 3.088416 & -2.236983 & 0.757918 \\
\hline 16 & 1 & 0 & 2.489840 & -1.613210 & 2.307397 \\
\hline 17 & 1 & 0 & 3.306516 & -0.531662 & 1.165207 \\
\hline 18 & 1 & 0 & 0.615947 & -1.501591 & 3.150719 \\
\hline 19 & 1 & 0 & -0.323342 & 1.371406 & 2.595836 \\
\hline 20 & 1 & 0 & 1.328259 & 0.859187 & 2.962902 \\
\hline 21 & 1 & 0 & -2.408060 & -2.521488 & 0.639290 \\
\hline 22 & 1 & 0 & -3.647549 & -0.173180 & 0.157773 \\
\hline 23 & 1 & 0 & -3.023700 & 0.597623 & -2.330383 \\
\hline 24 & 1 & 0 & -1.393812 & -1.273371 & -3.395396 \\
\hline 25 & 1 & 0 & -1.024424 & -3.198386 & -1.560150 \\
\hline 26 & 6 & 0 & 0.055265 & 1.343882 & -4.502558 \\
\hline 27 & 1 & 0 & 0.267327 & 0.343852 & -4.888008 \\
\hline 28 & 1 & 0 & -1.027580 & 1.501299 & -4.531259 \\
\hline
\end{tabular}




\begin{tabular}{|c|c|c|c|c|c|}
\hline 29 & 1 & 0 & 0.535615 & 2.078370 & -5.156100 \\
\hline 30 & 1 & 0 & -1.056794 & -1.057327 & 2.834175 \\
\hline 31 & 1 & 0 & 3.243533 & -2.390515 & -1.684400 \\
\hline 32 & 1 & 0 & 3.404785 & -0.025366 & -2.166660 \\
\hline 33 & 1 & 0 & 1.734016 & -1.391468 & -3.301230 \\
\hline 34 & 7 & 0 & 0.056325 & 0.712236 & 4.609874 \\
\hline 35 & 6 & 0 & 0.783584 & 1.844097 & 5.187262 \\
\hline 36 & 1 & 0 & 1.854600 & 1.735823 & 4.986353 \\
\hline 37 & 1 & 0 & 0.453805 & 2.823814 & 4.791220 \\
\hline 38 & 1 & 0 & 0.640573 & 1.850794 & 6.273374 \\
\hline 39 & 6 & 0 & -1.366581 & 0.810908 & 4.941019 \\
\hline 40 & 1 & 0 & -1.485555 & 0.820876 & 6.029745 \\
\hline 41 & 1 & 0 & -1.840918 & 1.723861 & 4.531022 \\
\hline 42 & 1 & 0 & -1.910682 & -0.056930 & 4.557111 \\
\hline 43 & 6 & 0 & 1.047210 & 2.330443 & -2.498337 \\
\hline 44 & 8 & 0 & 1.576618 & 3.116054 & -1.805186 \\
\hline 45 & 8 & 0 & -0.843811 & 1.436133 & -0.274196 \\
\hline
\end{tabular}

Results for the Model System $\mathrm{Ti}\left(\eta-\mathrm{C}_{5} \mathrm{H}_{5}\right)(\mathrm{NMe})\left\{\mathrm{MeC}\left(\mathrm{NCH}_{2} \mathrm{CH}_{2} \mathrm{NMe}_{2}\right)\left(\mathrm{NSiH}_{3}\right)\right\}$

Complex I: $\mathrm{E}=-792.010626779$

Input orientation:

\begin{tabular}{|c|c|c|c|c|c|}
\hline \multirow{2}{*}{$\begin{array}{l}\text { Center } \\
\text { Number }\end{array}$} & \multirow{2}{*}{$\begin{array}{l}\text { Atomic } \\
\text { Number }\end{array}$} & \multirow{2}{*}{$\begin{array}{l}\text { Atomic } \\
\text { Type }\end{array}$} & \multicolumn{3}{|c|}{ Coordinates (Angstroms) } \\
\hline & & & $\mathrm{X}$ & $\mathrm{Y}$ & Z \\
\hline & . & & ---- & --------- & --------- \\
\hline 1 & 22 & 0 & .270038 & .553316 & -1.472598 \\
\hline 2 & 7 & 0 & .454718 & -.777090 & .144752 \\
\hline 3 & 7 & 0 & 2.161336 & .489118 & -.549427 \\
\hline 4 & 7 & 0 & -.569884 & 1.912223 & -.936793 \\
\hline 5 & 6 & 0 & 1.695424 & -.377559 & .380831 \\
\hline 6 & 6 & 0 & 2.530352 & -.784881 & 1.568374 \\
\hline 7 & 6 & 0 & -.334755 & -1.607835 & 1.037904 \\
\hline 8 & 6 & 0 & -1.139803 & -.781914 & 2.054119 \\
\hline 9 & 14 & 0 & 3.658244 & 1.382130 & -.524318 \\
\hline 10 & 6 & 0 & -.690361 & .665844 & -3.627290 \\
\hline 11 & 6 & 0 & -1.082814 & -.574695 & -3.067868 \\
\hline 12 & 6 & 0 & .075877 & -1.389966 & -2.941690 \\
\hline 13 & 6 & 0 & 1.183314 & -.648107 & -3.399902 \\
\hline 14 & 6 & 0 & .713728 & .629697 & -3.809673 \\
\hline 15 & 1 & 0 & 3.557206 & -.992770 & 1.250423 \\
\hline 16 & 1 & 0 & 2.142351 & -1.669124 & 2.077011 \\
\hline 17 & 1 & 0 & 2.567364 & .035648 & 2.295494 \\
\hline 18 & 1 & 0 & .297729 & -2.337324 & 1.565458 \\
\hline 19 & 1 & 0 & -1.759274 & -.054780 & 1.516496 \\
\hline 20 & 1 & 0 & -.437189 & -.208098 & 2.671472 \\
\hline 21 & 1 & 0 & -2.092943 & -.850613 & -2.786889 \\
\hline 22 & 1 & 0 & -1.340219 & 1.505527 & -3.836648 \\
\hline 23 & 1 & 0 & 1.324026 & 1.440620 & -4.191417 \\
\hline 24 & 1 & 0 & 2.215752 & -.974050 & -3.396366 \\
\hline 25 & 1 & 0 & .106753 & -2.389926 & -2.527183 \\
\hline 26 & 6 & 0 & -1.402379 & 3.068729 & -.769391 \\
\hline 27 & 1 & 0 & -2.006488 & 3.271717 & -1.668008 \\
\hline 28 & 1 & 0 & -2.098372 & 2.940312 & .072998 \\
\hline 29 & 1 & 0 & -.801958 & 3.967587 & -.564702 \\
\hline 30 & 1 & 0 & -1.027873 & -2.190485 & .417020 \\
\hline 31 & 1 & 0 & 4.865361 & .481019 & -.540606 \\
\hline 32 & 1 & 0 & 3.796898 & 2.270594 & .682764 \\
\hline 33 & 1 & 0 & 3.686573 & 2.231987 & -1.754681 \\
\hline 34 & 6 & 0 & -2.037271 & -1.667532 & 2.922895 \\
\hline
\end{tabular}




$\begin{array}{rrrrrr}35 & 1 & 0 & -1.411239 & -2.350626 & 3.538592 \\ 36 & 1 & 0 & -2.641177 & -2.306273 & 2.263023 \\ 37 & 7 & 0 & -2.975557 & -.907224 & 3.755972 \\ 38 & 6 & 0 & -2.292408 & -.144692 & 4.800440 \\ 39 & 1 & 0 & -3.035210 & .400729 & 5.391954 \\ 40 & 1 & 0 & -1.706047 & -.785297 & 5.490077 \\ 41 & 1 & 0 & -1.617341 & .591272 & 4.355599 \\ 42 & 6 & 0 & -3.969330 & -1.799833 & 4.351216 \\ 43 & 1 & 0 & -4.513635 & -2.325553 & 3.559219 \\ 44 & 1 & 0 & -3.527621 & -2.557372 & 5.029072 \\ 45 & 1 & 0 & -4.691412 & -1.210198 & 4.926117 \\ - & 1 & & & & \end{array}$

$\mathrm{CO}_{2}$ Attack from the $\mathrm{SiH}_{3}$ side

TS I-II $: E=-980.513684722$

Input orientation:

\begin{tabular}{|c|c|c|c|c|c|}
\hline \multirow{2}{*}{$\begin{array}{l}\text { Center } \\
\text { Number }\end{array}$} & \multirow{2}{*}{$\begin{array}{l}\text { Atomic } \\
\text { Number }\end{array}$} & \multirow{2}{*}{$\begin{array}{c}\text { Atomic } \\
\text { Type }\end{array}$} & \multicolumn{3}{|c|}{ Coordinates (Angstroms) } \\
\hline & & & $\mathrm{X}$ & $\mathrm{Y}$ & Z \\
\hline--1 & & & --- & ---- & ------ \\
\hline 1 & 22 & 0 & -1.289060 & -0.449735 & -0.236910 \\
\hline 2 & 7 & 0 & 0.363918 & 0.712173 & -0.839597 \\
\hline 3 & 7 & 0 & -1.416700 & 1.676140 & 0.045230 \\
\hline 4 & 7 & 0 & -0.552162 & -1.331308 & 1.028726 \\
\hline 5 & 6 & 0 & -0.154975 & 1.846361 & -0.409431 \\
\hline 6 & 6 & 0 & 0.548172 & 3.179469 & -0.399488 \\
\hline 7 & 6 & 0 & 1.735033 & 0.533312 & -1.281316 \\
\hline 8 & 6 & 0 & 2.710752 & 0.260166 & -0.125666 \\
\hline 9 & 14 & 0 & -2.524828 & 2.874421 & 0.657717 \\
\hline 10 & 6 & 0 & -2.343536 & -2.341483 & -1.174804 \\
\hline 11 & 6 & 0 & -1.127060 & -2.144666 & -1.875754 \\
\hline 12 & 6 & 0 & -1.239840 & -0.935272 & -2.607227 \\
\hline 13 & 6 & 0 & -2.516028 & -0.380905 & -2.359251 \\
\hline 14 & 6 & 0 & -3.196115 & -1.249989 & -1.472315 \\
\hline 15 & 1 & 0 & -0.045452 & 3.922175 & -0.942625 \\
\hline 16 & 1 & 0 & 1.541725 & 3.135583 & -0.848348 \\
\hline 17 & 1 & 0 & 0.655032 & 3.537820 & 0.630916 \\
\hline 18 & 1 & 0 & 2.077006 & 1.403071 & -1.863579 \\
\hline 19 & 1 & 0 & 2.371647 & -0.622031 & 0.427866 \\
\hline 20 & 1 & 0 & 2.676408 & 1.108093 & 0.570104 \\
\hline 21 & 1 & 0 & -0.263970 & -2.798268 & -1.846174 \\
\hline 22 & 1 & 0 & -2.571804 & -3.171612 & -0.518008 \\
\hline 23 & 1 & 0 & -4.185016 & -1.088319 & -1.060931 \\
\hline 24 & 1 & 0 & -2.891055 & 0.558411 & -2.745346 \\
\hline 25 & 1 & 0 & -0.468013 & -0.492052 & -3.223077 \\
\hline 26 & 6 & 0 & 0.114795 & -2.357166 & 1.779462 \\
\hline 27 & 1 & 0 & -0.032367 & -3.351873 & 1.329514 \\
\hline 28 & 1 & 0 & 1.197384 & -2.168317 & 1.821279 \\
\hline 29 & 1 & 0 & -0.248409 & -2.402167 & 2.815886 \\
\hline 30 & 1 & 0 & 1.746689 & -0.322377 & -1.969205 \\
\hline 31 & 1 & 0 & -2.313231 & 4.219262 & 0.015201 \\
\hline 32 & 1 & 0 & -2.403389 & 3.076475 & 2.144809 \\
\hline 33 & 1 & 0 & -3.924817 & 2.436052 & 0.365237 \\
\hline 34 & 6 & 0 & 4.137149 & 0.020625 & -0.628359 \\
\hline 35 & 1 & 0 & 4.533448 & 0.947723 & -1.097862 \\
\hline 36 & 1 & 0 & 4.105882 & -0.742276 & -1.419084 \\
\hline 37 & 7 & 0 & 5.042188 & -0.480821 & 0.412196 \\
\hline 38 & 6 & 0 & 5.303622 & 0.521931 & 1.444459 \\
\hline 39 & 1 & 0 & 5.981186 & 0.099217 & 2.193725 \\
\hline 40 & 1 & 0 & 5.767656 & 1.445372 & 1.042797 \\
\hline
\end{tabular}




\begin{tabular}{|c|c|c|c|c|c|}
\hline 41 & 1 & 0 & 4.375672 & 0.796273 & 1.953319 \\
\hline 42 & 6 & 0 & 6.300344 & -0.935956 & -0.178432 \\
\hline 43 & 1 & 0 & 6.098852 & -1.722303 & -0.913927 \\
\hline 44 & 1 & 0 & 6.865850 & -0.127019 & -0.682632 \\
\hline 45 & 1 & 0 & 6.938848 & -1.358320 & 0.604876 \\
\hline 46 & 6 & 0 & -2.611504 & -0.787658 & 2.251225 \\
\hline 47 & 8 & 0 & -3.132165 & -0.253923 & 1.325592 \\
\hline 48 & 8 & 0 & -2.355884 & -1.255104 & 3.288752 \\
\hline
\end{tabular}

Complex II: $\mathrm{E}=-980.555144262$

Z-Matrix orientation:

\begin{tabular}{|c|c|c|c|c|c|}
\hline \multirow{2}{*}{$\begin{array}{l}\text { Center } \\
\text { Number }\end{array}$} & \multirow{2}{*}{$\begin{array}{l}\text { Atomic } \\
\text { Number }\end{array}$} & \multirow{2}{*}{$\begin{array}{l}\text { Atomic } \\
\text { Type }\end{array}$} & \multicolumn{3}{|c|}{ Coordinates (Angstroms) } \\
\hline & & & $\mathrm{X}$ & $\mathrm{Y}$ & $\mathrm{Z}$ \\
\hline---1 & --- & - & -------- & -------- & -------- \\
\hline 1 & 22 & 0 & -1.458019 & -0.389016 & -0.212883 \\
\hline 2 & 7 & 0 & 0.332672 & 0.616303 & -0.780455 \\
\hline 3 & 7 & 0 & -1.497881 & 1.670278 & -0.179901 \\
\hline 4 & 7 & 0 & -0.839123 & -1.144443 & 1.425056 \\
\hline 5 & 6 & 0 & -0.199788 & 1.799787 & -0.525603 \\
\hline 6 & 6 & 0 & 0.504490 & 3.126529 & -0.593203 \\
\hline 7 & 6 & 0 & 1.689066 & 0.403166 & -1.260082 \\
\hline 8 & 6 & 0 & 2.712495 & 0.236047 & -0.126184 \\
\hline 9 & 14 & 0 & -2.597648 & 2.787586 & 0.622728 \\
\hline 10 & 6 & 0 & -2.068410 & -2.496697 & -1.095327 \\
\hline 11 & 6 & 0 & -0.965859 & -2.050260 & -1.860961 \\
\hline 12 & 6 & 0 & -1.365135 & -0.887960 & -2.570433 \\
\hline 13 & 6 & 0 & -2.709714 & -0.610067 & -2.237285 \\
\hline 14 & 6 & 0 & -3.142690 & -1.599430 & -1.316748 \\
\hline 15 & 1 & 0 & -0.073120 & 3.828557 & -1.202373 \\
\hline 16 & 1 & 0 & 1.511977 & 3.047222 & -1.004460 \\
\hline 17 & 1 & 0 & 0.581585 & 3.556519 & 0.412412 \\
\hline 18 & 1 & 0 & 2.000160 & 1.222800 & -1.925300 \\
\hline 19 & 1 & 0 & 2.419759 & -0.607010 & 0.509473 \\
\hline 20 & 1 & 0 & 2.689753 & 1.134454 & 0.503265 \\
\hline 21 & 1 & 0 & 0.018748 & -2.502494 & -1.880174 \\
\hline 22 & 1 & 0 & -2.078287 & -3.341963 & -0.419045 \\
\hline 23 & 1 & 0 & -4.105552 & -1.633315 & -0.824790 \\
\hline 24 & 1 & 0 & -3.292277 & 0.234224 & -2.584010 \\
\hline 25 & 1 & 0 & -0.735964 & -0.294298 & -3.220563 \\
\hline 26 & 6 & 0 & 0.170168 & -1.944528 & 2.095567 \\
\hline 27 & 1 & 0 & 0.851032 & -2.378186 & 1.357615 \\
\hline 28 & 1 & 0 & 0.758611 & -1.332623 & 2.790452 \\
\hline 29 & 1 & 0 & -0.296371 & -2.749727 & 2.674154 \\
\hline 30 & 1 & 0 & 1.680741 & -0.507040 & -1.874399 \\
\hline 31 & 1 & 0 & -2.345586 & 4.178577 & 0.104009 \\
\hline 32 & 1 & 0 & -2.405892 & 2.816531 & 2.107812 \\
\hline 33 & 1 & 0 & -3.995115 & 2.398713 & 0.279444 \\
\hline 34 & 6 & 0 & 4.123201 & -0.017983 & -0.667022 \\
\hline 35 & 1 & 0 & 4.487107 & 0.882765 & -1.208232 \\
\hline 36 & 1 & 0 & 4.074594 & -0.829569 & -1.406875 \\
\hline 37 & 7 & 0 & 5.069611 & -0.441253 & 0.369951 \\
\hline 38 & 6 & 0 & 5.351443 & 0.628109 & 1.327832 \\
\hline 39 & 1 & 0 & 6.064755 & 0.263504 & 2.074022 \\
\hline 40 & 1 & 0 & 5.781971 & 1.532107 & 0.852183 \\
\hline 41 & 1 & 0 & 4.439155 & 0.917944 & 1.856275 \\
\hline 42 & 6 & 0 & 6.312961 & -0.919591 & -0.234678 \\
\hline 43 & 1 & 0 & 6.097120 & -1.755441 & -0.908984 \\
\hline 44 & 1 & 0 & 6.848961 & -0.139385 & -0.810559 \\
\hline 45 & 1 & 0 & 6.983651 & -1.281772 & 0.551512 \\
\hline
\end{tabular}




$\begin{array}{rrrrrr}46 & 6 & 0 & -2.040429 & -0.854834 & 2.077974 \\ 47 & 8 & 0 & -2.833518 & -0.258524 & 1.171633 \\ 48 & 8 & 0 & -2.317205 & -1.105084 & 3.232958\end{array}$

TS II-III $: E=-980.523191019$

Input orientation:

\begin{tabular}{|c|c|c|c|c|c|}
\hline \multirow{2}{*}{$\begin{array}{l}\text { Center } \\
\text { Number }\end{array}$} & \multirow{2}{*}{$\begin{array}{l}\text { Atomic } \\
\text { Number }\end{array}$} & \multirow{2}{*}{$\begin{array}{l}\text { Atomic } \\
\text { Type }\end{array}$} & \multicolumn{3}{|c|}{ Coordinates (Angstroms) } \\
\hline & & & $\mathrm{X}$ & $\mathrm{Y}$ & $\mathrm{Z}$ \\
\hline & & & -- & --- & -------- \\
\hline 1 & 22 & 0 & -0.344149 & -0.067166 & -1.556433 \\
\hline 2 & 7 & 0 & 0.542511 & -0.845086 & 0.243493 \\
\hline 3 & 7 & 0 & 1.742650 & -0.512456 & -1.576434 \\
\hline 4 & 7 & 0 & -1.891417 & 0.725849 & -0.185688 \\
\hline 5 & 6 & 0 & 1.764832 & -0.815765 & -0.269595 \\
\hline 6 & 6 & 0 & 3.044891 & -1.058085 & 0.488213 \\
\hline 7 & 6 & 0 & 0.271485 & -1.121735 & 1.645647 \\
\hline 8 & 6 & 0 & 0.441188 & 0.102980 & 2.559680 \\
\hline 9 & 14 & 0 & 3.073681 & -0.016578 & -2.594147 \\
\hline 10 & 6 & 0 & -1.922970 & -0.400985 & -3.288415 \\
\hline 11 & 6 & 0 & -2.307787 & -1.329535 & -2.288970 \\
\hline 12 & 6 & 0 & -1.295348 & -2.313707 & -2.178039 \\
\hline 13 & 6 & 0 & -0.276572 & -1.983146 & -3.096371 \\
\hline 14 & 6 & 0 & -0.661020 & -0.801169 & -3.783333 \\
\hline 15 & 1 & 0 & 3.525899 & -0.100695 & 0.724123 \\
\hline 16 & 1 & 0 & 3.741471 & -1.635584 & -0.126940 \\
\hline 17 & 1 & 0 & 2.884233 & -1.593610 & 1.426170 \\
\hline 18 & 1 & 0 & 0.905596 & -1.945450 & 2.010352 \\
\hline 19 & 1 & 0 & -0.220062 & 0.906887 & 2.215592 \\
\hline 20 & 1 & 0 & 1.468274 & 0.476136 & 2.464110 \\
\hline 21 & 1 & 0 & -3.223650 & -1.290078 & -1.712180 \\
\hline 22 & 1 & 0 & -2.474327 & 0.480078 & -3.591454 \\
\hline 23 & 1 & 0 & -0.078528 & -0.280336 & -4.532960 \\
\hline 24 & 1 & 0 & 0.657595 & -2.513003 & -3.225498 \\
\hline 25 & 1 & 0 & -1.283274 & -3.145376 & -1.484338 \\
\hline 26 & 6 & 0 & -2.910147 & 0.246806 & 0.768539 \\
\hline 27 & 1 & 0 & -2.957963 & -0.839322 & 0.686460 \\
\hline 28 & 1 & 0 & -2.642325 & 0.513243 & 1.795684 \\
\hline 29 & 1 & 0 & -3.897383 & 0.661056 & 0.539247 \\
\hline 30 & 1 & 0 & -0.761910 & -1.482615 & 1.717388 \\
\hline 31 & 1 & 0 & 4.165124 & -1.055048 & -2.619471 \\
\hline 32 & 1 & 0 & 3.707460 & 1.274265 & -2.156092 \\
\hline 33 & 1 & 0 & 2.543848 & 0.143248 & -3.982094 \\
\hline 34 & 6 & 0 & 0.114873 & -0.222613 & 4.020584 \\
\hline 35 & 1 & 0 & 0.893916 & -0.897736 & 4.437328 \\
\hline 36 & 1 & 0 & -0.831988 & -0.780991 & 4.055927 \\
\hline 37 & 7 & 0 & -0.064282 & 0.970979 & 4.854443 \\
\hline 38 & 6 & 0 & 1.174344 & 1.738184 & 4.989120 \\
\hline 39 & 1 & 0 & 0.989754 & 2.610160 & 5.624946 \\
\hline 40 & 1 & 0 & 1.999390 & 1.150963 & 5.439997 \\
\hline 41 & 1 & 0 & 1.503738 & 2.103619 & 4.012798 \\
\hline 42 & 6 & 0 & -0.575054 & 0.599770 & 6.173832 \\
\hline 43 & 1 & 0 & -1.528565 & 0.071254 & 6.065093 \\
\hline 44 & 1 & 0 & 0.117279 & -0.051167 & 6.743872 \\
\hline 45 & 1 & 0 & -0.752975 & 1.505109 & 6.763715 \\
\hline 46 & 6 & 0 & -1.774536 & 1.955396 & -0.414980 \\
\hline 47 & 8 & 0 & -0.189838 & 1.567512 & -1.823088 \\
\hline 48 & 8 & 0 & -1.903436 & 3.116410 & -0.431709 \\
\hline
\end{tabular}




\begin{tabular}{|c|c|c|c|c|c|}
\hline \multirow{2}{*}{$\begin{array}{l}\text { Center } \\
\text { Number }\end{array}$} & \multirow{2}{*}{$\begin{array}{l}\text { Atomic } \\
\text { Number }\end{array}$} & \multirow{2}{*}{$\begin{array}{c}\text { Atomic } \\
\text { Type }\end{array}$} & \multicolumn{3}{|c|}{ Coordinates (Angstroms) } \\
\hline & & & $\mathrm{X}$ & $\mathrm{Y}$ & Z \\
\hline- & & 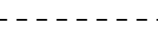 & -------- & --------- & ------- \\
\hline 1 & 22 & 0 & -1.553463 & -0.360323 & 0.182381 \\
\hline 2 & 7 & 0 & 0.078106 & 0.721155 & -0.744338 \\
\hline 3 & 7 & 0 & -1.689094 & 1.765749 & 0.080003 \\
\hline 4 & 7 & 0 & 0.045087 & -2.057111 & 0.281769 \\
\hline 5 & 6 & 0 & -0.452163 & 1.894642 & -0.423208 \\
\hline 6 & 6 & 0 & 0.227494 & 3.234609 & -0.554539 \\
\hline 7 & 6 & 0 & 1.418202 & 0.587193 & -1.295078 \\
\hline 8 & 6 & 0 & 2.522179 & 0.525730 & -0.226551 \\
\hline 9 & 14 & 0 & -2.596618 & 2.961450 & 0.975527 \\
\hline 10 & 6 & 0 & -3.431542 & -1.821091 & 0.019527 \\
\hline 11 & 6 & 0 & -2.590549 & -2.219227 & -1.045452 \\
\hline 12 & 6 & 0 & -2.549177 & -1.170369 & -2.003349 \\
\hline 13 & 6 & 0 & -3.344699 & -0.117062 & -1.515227 \\
\hline 14 & 6 & 0 & -3.882398 & -0.511144 & -0.259152 \\
\hline 15 & 1 & 0 & 0.600937 & 3.558113 & 0.425086 \\
\hline 16 & 1 & 0 & -0.488980 & 3.985928 & -0.900188 \\
\hline 17 & 1 & 0 & 1.071919 & 3.218166 & -1.246232 \\
\hline 18 & 1 & 0 & 1.634703 & 1.404630 & -2.000454 \\
\hline 19 & 1 & 0 & 2.334090 & -0.323133 & 0.441328 \\
\hline 20 & 1 & 0 & 2.468139 & 1.431328 & 0.390162 \\
\hline 21 & 1 & 0 & -2.086213 & -3.174571 & -1.123124 \\
\hline 22 & 1 & 0 & -3.647863 & -2.392584 & 0.912864 \\
\hline 23 & 1 & 0 & -4.516820 & 0.090203 & 0.379692 \\
\hline 24 & 1 & 0 & -3.478100 & 0.849615 & -1.982935 \\
\hline 25 & 1 & 0 & -1.983933 & -1.166223 & -2.927925 \\
\hline 26 & 6 & 0 & 0.724371 & -2.821102 & -0.784088 \\
\hline 27 & 1 & 0 & 0.306498 & -2.500178 & -1.737520 \\
\hline 28 & 1 & 0 & 1.799921 & -2.622950 & -0.778810 \\
\hline 29 & 1 & 0 & 0.555468 & -3.896041 & -0.668665 \\
\hline 30 & 1 & 0 & 1.444956 & -0.333941 & -1.890193 \\
\hline 31 & 1 & 0 & -2.868126 & 4.186274 & 0.140680 \\
\hline 32 & 1 & 0 & -1.892458 & 3.429869 & 2.219070 \\
\hline 33 & 1 & 0 & -3.907120 & 2.353890 & 1.354557 \\
\hline 34 & 6 & 0 & 3.914167 & 0.369303 & -0.847654 \\
\hline 35 & 1 & 0 & 4.214345 & 1.318828 & -1.341723 \\
\hline 36 & 1 & 0 & 3.865538 & -0.391787 & -1.640407 \\
\hline 37 & 7 & 0 & 4.927742 & -0.082465 & 0.111977 \\
\hline 38 & 6 & 0 & 5.172114 & 0.909538 & 1.159762 \\
\hline 39 & 1 & 0 & 5.932633 & 0.526307 & 1.847911 \\
\hline 40 & 1 & 0 & 5.526361 & 1.881036 & 0.760572 \\
\hline 41 & 1 & 0 & 4.260271 & 1.084942 & 1.736825 \\
\hline 42 & 6 & 0 & 6.175974 & -0.406798 & -0.578054 \\
\hline 43 & 1 & 0 & 5.992439 & -1.186249 & -1.325897 \\
\hline 44 & 1 & 0 & 6.630838 & 0.463439 & -1.091459 \\
\hline 45 & 1 & 0 & 6.902646 & -0.792590 & 0.144752 \\
\hline 46 & 6 & 0 & 0.236791 & -2.285453 & 1.483748 \\
\hline 47 & 8 & 0 & -1.504283 & -0.469383 & 1.814076 \\
\hline 48 & 8 & 0 & 0.439192 & -2.528044 & 2.603709 \\
\hline
\end{tabular}

TS III-IV $_{\text {IV }}: E=-980.520792426$

Input orientation: 


\begin{tabular}{|c|c|c|c|c|c|}
\hline \multirow{2}{*}{$\begin{array}{l}\text { Center } \\
\text { Number }\end{array}$} & \multirow{2}{*}{$\begin{array}{l}\text { Atomic } \\
\text { Number }\end{array}$} & \multirow{2}{*}{$\begin{array}{c}\text { Atomic } \\
\text { Type }\end{array}$} & \multicolumn{3}{|c|}{ Coordinates (Angstroms) } \\
\hline & & & $\mathrm{X}$ & $\mathrm{Y}$ & $\mathrm{Z}$ \\
\hline & & & & --- & -------- \\
\hline 1 & 22 & 0 & -0.226165 & 0.213948 & -1.665732 \\
\hline 2 & 7 & 0 & 0.786129 & -0.647442 & -0.024530 \\
\hline 3 & 7 & 0 & 1.863749 & 0.245626 & -1.758667 \\
\hline 4 & 7 & 0 & -2.535557 & 0.148082 & 0.466843 \\
\hline 5 & 6 & 0 & 1.962915 & -0.296394 & -0.527054 \\
\hline 6 & 6 & 0 & 3.291142 & -0.445852 & 0.168862 \\
\hline 7 & 6 & 0 & 0.613681 & -1.226671 & 1.299197 \\
\hline 8 & 6 & 0 & 0.573950 & -0.181676 & 2.424818 \\
\hline 9 & 14 & 0 & 3.104304 & 1.079554 & -2.665756 \\
\hline 10 & 6 & 0 & -1.526307 & 0.029219 & -3.640189 \\
\hline 11 & 6 & 0 & -2.143734 & -0.781334 & -2.660653 \\
\hline 12 & 6 & 0 & -1.294234 & -1.897804 & -2.409394 \\
\hline 13 & 6 & 0 & -0.150087 & -1.763505 & -3.217421 \\
\hline 14 & 6 & 0 & -0.278542 & -0.558575 & -3.958800 \\
\hline 15 & 1 & 0 & 3.655641 & 0.539918 & 0.482798 \\
\hline 16 & 1 & 0 & 4.029839 & -0.867014 & -0.520904 \\
\hline 17 & 1 & 0 & 3.238217 & -1.083470 & 1.052682 \\
\hline 18 & 1 & 0 & 1.401473 & -1.968867 & 1.502997 \\
\hline 19 & 1 & 0 & -0.259479 & 0.506261 & 2.244825 \\
\hline 20 & 1 & 0 & 1.495205 & 0.412968 & 2.390770 \\
\hline 21 & 1 & 0 & -3.096607 & -0.581324 & -2.187702 \\
\hline 22 & 1 & 0 & -1.909082 & 0.967039 & -4.021397 \\
\hline 23 & 1 & 0 & 0.453206 & -0.157445 & -4.649835 \\
\hline 24 & 1 & 0 & 0.712309 & -2.418520 & -3.219207 \\
\hline 25 & 1 & 0 & -1.482560 & -2.698074 & -1.703054 \\
\hline 26 & 6 & 0 & -3.281601 & -0.992009 & 0.988109 \\
\hline 27 & 1 & 0 & -3.000582 & -1.880114 & 0.419766 \\
\hline 28 & 1 & 0 & -3.040754 & -1.164719 & 2.042365 \\
\hline 29 & 1 & 0 & -4.362942 & -0.849135 & 0.893869 \\
\hline 30 & 1 & 0 & -0.333499 & -1.778410 & 1.290449 \\
\hline 31 & 1 & 0 & 4.260738 & 0.171584 & -2.990122 \\
\hline 32 & 1 & 0 & 3.666016 & 2.263143 & -1.928549 \\
\hline 33 & 1 & 0 & 2.486523 & 1.542174 & -3.944517 \\
\hline 34 & 6 & 0 & 0.400067 & -0.837574 & 3.798640 \\
\hline 35 & 1 & 0 & 1.330198 & -1.381536 & 4.074781 \\
\hline 36 & 1 & 0 & -0.394624 & -1.594955 & 3.731699 \\
\hline 37 & 7 & 0 & -0.001246 & 0.106461 & 4.846670 \\
\hline 38 & 6 & 0 & 1.030273 & 1.110263 & 5.108461 \\
\hline 39 & 1 & 0 & 0.683114 & 1.784101 & 5.898467 \\
\hline 40 & 1 & 0 & 1.993494 & 0.666893 & 5.432225 \\
\hline 41 & 1 & 0 & 1.209637 & 1.712800 & 4.214116 \\
\hline 42 & 6 & 0 & -0.335808 & -0.609284 & 6.077118 \\
\hline 43 & 1 & 0 & -1.144844 & -1.321791 & 5.881453 \\
\hline 44 & 1 & 0 & 0.520038 & -1.168436 & 6.505010 \\
\hline 45 & 1 & 0 & -0.685854 & 0.104422 & 6.830344 \\
\hline 46 & 6 & 0 & -2.716721 & 1.340948 & 0.649899 \\
\hline 47 & 8 & 0 & -0.627831 & 1.762993 & -1.406878 \\
\hline 48 & 8 & 0 & -2.818533 & 2.501293 & 0.772075 \\
\hline
\end{tabular}

Complex oxo IV: $E=-772.632470936$

Input orientation:

\begin{tabular}{|c|c|c|c|c|c|}
\hline \multirow{2}{*}{$\begin{array}{l}\text { Center } \\
\text { Number }\end{array}$} & \multirow{2}{*}{$\begin{array}{l}\text { Atomic } \\
\text { Number }\end{array}$} & \multirow{2}{*}{$\begin{array}{l}\text { Atomic } \\
\text { Type }\end{array}$} & \multicolumn{3}{|c|}{ Coordinates (Angstroms) } \\
\hline & & & $\mathrm{X}$ & $\mathrm{Y}$ & Z \\
\hline \multicolumn{6}{|c|}{ 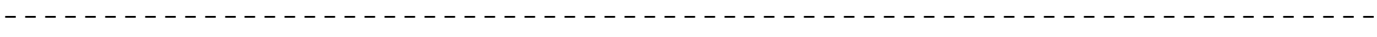 } \\
\hline 1 & 22 & 0 & -.592950 & .402798 & -1.466202 \\
\hline 2 & 7 & 0 & .378234 & -.663357 & .038404 \\
\hline
\end{tabular}




\begin{tabular}{|c|c|c|c|c|c|}
\hline 3 & 7 & 0 & 1.459934 & .182207 & -1.724547 \\
\hline 4 & 8 & 0 & -.901844 & 1.891187 & -.902275 \\
\hline 5 & 6 & 0 & 1.558277 & -.423163 & -.516428 \\
\hline 6 & 6 & 0 & 2.893848 & -.738310 & .104289 \\
\hline 7 & 6 & 0 & .173484 & -1.172754 & 1.385145 \\
\hline 8 & 6 & 0 & .013251 & -.049207 & 2.421773 \\
\hline 9 & 14 & 0 & 2.751494 & .796785 & -2.731681 \\
\hline 10 & 6 & 0 & -2.597891 & .316665 & -2.704961 \\
\hline 11 & 6 & 0 & -2.641687 & -.769556 & -1.799204 \\
\hline 12 & 6 & 0 & -1.660755 & -1.722581 & -2.198772 \\
\hline 13 & 6 & 0 & -.989884 & -1.209491 & -3.323789 \\
\hline 14 & 6 & 0 & -1.550544 & .064548 & -3.624140 \\
\hline 15 & 1 & 0 & 3.586824 & -1.100498 & -.661644 \\
\hline 16 & 1 & 0 & 2.827353 & -1.491802 & .890815 \\
\hline 17 & 1 & 0 & 3.324417 & .170939 & .541067 \\
\hline 18 & 1 & 0 & .994249 & -1.841944 & 1.681070 \\
\hline 19 & 1 & 0 & -.832384 & .589522 & 2.140485 \\
\hline 20 & 1 & 0 & .910886 & .581125 & 2.396039 \\
\hline 21 & 1 & 0 & -3.317117 & -.862809 & -.955847 \\
\hline 22 & 1 & 0 & -3.200827 & 1.213671 & -2.654740 \\
\hline 23 & 1 & 0 & -1.240416 & .724465 & -4.426972 \\
\hline 24 & 1 & 0 & -.157920 & -1.673646 & -3.838473 \\
\hline 25 & 1 & 0 & -1.440459 & -2.655448 & -1.694575 \\
\hline 26 & 1 & 0 & -.737945 & -1.783992 & 1.370260 \\
\hline 27 & 1 & 0 & 3.649110 & -.306289 & -3.223677 \\
\hline 28 & 1 & 0 & 3.621257 & 1.792704 & -2.017129 \\
\hline 29 & 1 & 0 & 2.107128 & 1.455497 & -3.907931 \\
\hline 30 & 6 & 0 & -.223366 & -.610591 & 3.827255 \\
\hline 31 & 1 & 0 & .693095 & -1.133158 & 4.180972 \\
\hline 32 & 1 & 0 & -1.016377 & -1.370190 & 3.774421 \\
\hline 33 & 7 & 0 & -.668234 & .401710 & 4.789360 \\
\hline 34 & 6 & 0 & .357971 & 1.412602 & 5.043109 \\
\hline 35 & 1 & 0 & -.022840 & 2.135512 & 5.771864 \\
\hline 36 & 1 & 0 & 1.299101 & .983563 & 5.442966 \\
\hline 37 & 1 & 0 & .589202 & 1.959095 & 4.124927 \\
\hline 38 & 6 & 0 & -1.079753 & -.230560 & 6.041787 \\
\hline 39 & 1 & 0 & -1.886310 & -.945470 & 5.845888 \\
\hline 40 & 1 & 0 & -.256447 & -.769427 & 6.552097 \\
\hline 41 & 1 & 0 & -1.462078 & .532994 & 6.727402 \\
\hline
\end{tabular}

$\underline{\mathrm{CO}}_{2}$ attack from the $\mathrm{CH}_{2} \underline{\mathrm{CH}}_{2} \underline{\mathrm{CH}}_{2} \underline{\mathrm{NMe}}_{2}$ side

TS $_{\text {I-II }}: E=-980.513089355$

Input orientation:

\begin{tabular}{|c|c|c|c|c|c|}
\hline \multirow{2}{*}{$\begin{array}{l}\text { Center } \\
\text { Number }\end{array}$} & \multirow{2}{*}{$\begin{array}{l}\text { Atomic } \\
\text { Number }\end{array}$} & \multirow{2}{*}{$\begin{array}{l}\text { Atomic } \\
\text { Type }\end{array}$} & \multicolumn{3}{|c|}{ Coordinates (Angstroms) } \\
\hline & & & $\mathrm{X}$ & $\mathrm{Y}$ & $\mathrm{Z}$ \\
\hline \multicolumn{6}{|c|}{ - - - - - - - - - - - - - - - - - - - - - - - - - - - - - - - - - - - - - - - - - - - - - - - - - - - - - - - - - - - - - } \\
\hline 1 & 22 & 0 & -1.505127 & -0.413945 & -0.066519 \\
\hline 2 & 7 & 0 & 0.254300 & 0.616327 & -0.726684 \\
\hline 3 & 7 & 0 & -1.502018 & 1.706039 & 0.064667 \\
\hline 4 & 7 & 0 & -1.560848 & -0.780435 & 1.599589 \\
\hline 5 & 6 & 0 & -0.240098 & 1.798838 & -0.394929 \\
\hline 6 & 6 & 0 & 0.493240 & 3.112912 & -0.484502 \\
\hline 7 & 6 & 0 & 1.582417 & 0.420256 & -1.289006 \\
\hline 8 & 6 & 0 & 2.696057 & 0.369345 & -0.229854 \\
\hline 9 & 14 & 0 & -2.421129 & 2.882233 & 0.962573 \\
\hline 10 & 6 & 0 & -3.084223 & -2.038300 & -0.728174 \\
\hline 11 & 6 & 0 & -2.257989 & -1.844484 & -1.863071 \\
\hline 12 & 6 & 0 & -2.449616 & -0.523872 & -2.333132 \\
\hline
\end{tabular}




\begin{tabular}{|c|c|c|c|c|c|}
\hline 13 & 6 & 0 & -3.396735 & 0.099195 & -1.486945 \\
\hline 14 & 6 & 0 & -3.791736 & -0.831479 & -0.495246 \\
\hline 15 & 1 & 0 & -0.183508 & 3.900526 & -0.828950 \\
\hline 16 & 1 & 0 & 1.348575 & 3.068250 & -1.161967 \\
\hline 17 & 1 & 0 & 0.864419 & 3.403235 & 0.506406 \\
\hline 18 & 1 & 0 & 1.813700 & 1.201781 & -2.030142 \\
\hline 19 & 1 & 0 & 2.481610 & -0.430575 & 0.488140 \\
\hline 20 & 1 & 0 & 2.695362 & 1.311629 & 0.332130 \\
\hline 21 & 1 & 0 & -1.567898 & -2.571195 & -2.274496 \\
\hline 22 & 1 & 0 & -3.159733 & -2.943215 & -0.137997 \\
\hline 23 & 1 & 0 & -4.495413 & -0.650278 & 0.307533 \\
\hline 24 & 1 & 0 & -3.733798 & 1.124267 & -1.562391 \\
\hline 25 & 1 & 0 & -1.935794 & -0.061360 & -3.166451 \\
\hline 26 & 6 & 0 & -2.008793 & -1.074233 & 2.931140 \\
\hline 27 & 1 & 0 & -2.123007 & -0.148115 & 3.512702 \\
\hline 28 & 1 & 0 & -2.981874 & -1.589975 & 2.928168 \\
\hline 29 & 1 & 0 & -1.293744 & -1.713915 & 3.467445 \\
\hline 30 & 1 & 0 & 1.567164 & -0.530182 & -1.835425 \\
\hline 31 & 1 & 0 & -2.512614 & 4.203774 & 0.244999 \\
\hline 32 & 1 & 0 & -1.853568 & 3.168185 & 2.327010 \\
\hline 33 & 1 & 0 & -3.805945 & 2.341006 & 1.125845 \\
\hline 34 & 6 & 0 & 4.067220 & 0.111507 & -0.861771 \\
\hline 35 & 1 & 0 & 4.333514 & 0.952003 & -1.539728 \\
\hline 36 & 1 & 0 & 3.996340 & -0.787156 & -1.490376 \\
\hline 37 & 7 & 0 & 5.125766 & -0.136749 & 0.123410 \\
\hline 38 & 6 & 0 & 5.450116 & 1.061552 & 0.896301 \\
\hline 39 & 1 & 0 & 6.240548 & 0.823231 & 1.615685 \\
\hline 40 & 1 & 0 & 5.801675 & 1.901894 & 0.263987 \\
\hline 41 & 1 & 0 & 4.577333 & 1.398039 & 1.462329 \\
\hline 42 & 6 & 0 & 6.326257 & -0.653813 & -0.532877 \\
\hline 43 & 1 & 0 & 6.085879 & -1.582203 & -1.062152 \\
\hline 44 & 1 & 0 & 6.767805 & 0.055335 & -1.261250 \\
\hline 45 & 1 & 0 & 7.085811 & -0.880143 & 0.223065 \\
\hline 46 & 6 & 0 & 0.134699 & -2.478790 & 1.007011 \\
\hline 47 & 8 & 0 & 0.143013 & -2.209651 & -0.150076 \\
\hline 48 & 8 & 0 & 0.313295 & -2.942524 & 2.062168 \\
\hline
\end{tabular}

Complex II: $E=-980.5563869522598$

$\mathrm{Ti}-2.60799108 \mathrm{E}+00-1.08867175 \mathrm{E}+00 \quad-2.62207399 \mathrm{E}-01$

$\begin{array}{lrrr}\mathrm{N} & 2.99550612 \mathrm{E}-01 & 1.07447712 \mathrm{E}+00 & -1.66607261 \mathrm{E}+00 \\ \mathrm{~N} & -2.95339359 \mathrm{E}+00 & 2.95900952 \mathrm{E}+00 & 1.41999223 \mathrm{E}-01 \\ \mathrm{~N} & -2.26806176 \mathrm{E}+00 & -1.31909650 \mathrm{E}+00 & 3.35481655 \mathrm{E}+00 \\ \mathrm{C} & -6.48741668 \mathrm{E}-01 & 3.26154336 \mathrm{E}+00 & -8.77169709 \mathrm{E}-01 \\ \mathrm{C} & 6.63522385 \mathrm{E}-01 & 5.77789683 \mathrm{E}+00 & -1.05115296 \mathrm{E}+00 \\ \mathrm{C} & 2.83384592 \mathrm{E}+00 & 6.87878025 \mathrm{E}-01 & -2.66917361 \mathrm{E}+00 \\ \mathrm{C} & 4.85540869 \mathrm{E}+00 & 4.08012629 \mathrm{E}-01 & -6.03583556 \mathrm{E}-01 \\ \mathrm{Si} & -4.95264273 \mathrm{E}+00 & 5.28501517 \mathrm{E}+00 & 1.36372040 \mathrm{E}+00 \\ \mathrm{C} & -5.14876246 \mathrm{E}+00 & -4.40903597 \mathrm{E}+00 & -1.79901425 \mathrm{E}+00 \\ \mathrm{C} & -3.68695955 \mathrm{E}+00 & -3.61625678 \mathrm{E}+00 & -3.89194024 \mathrm{E}+00 \\ \mathrm{C} & -4.39071627 \mathrm{E}+00 & -1.11084156 \mathrm{E}+00 & -4.49457223 \mathrm{E}+00 \\ \mathrm{C} & -6.29104026 \mathrm{E}+00 & -3.51875450 \mathrm{E}-01 & -2.77244830 \mathrm{E}+00 \\ \mathrm{C} & -6.76061791 \mathrm{E}+00 & -2.38940787 \mathrm{E}+00 & -1.09368914 \mathrm{E}+00 \\ \mathrm{H} & -6.55218198 \mathrm{E}-01 & 7.22608658 \mathrm{E}+00 & -1.72023494 \mathrm{E}+00 \\ \mathrm{H} & 2.28766381 \mathrm{E}+00 & 5.73650775 \mathrm{E}+00 & -2.32149249 \mathrm{E}+00 \\ \mathrm{H} & 1.32846353 \mathrm{E}+00 & 6.35555241 \mathrm{E}+00 & 8.24433602 \mathrm{E}-01 \\ \mathrm{H} & 3.34070774 \mathrm{E}+00 & 2.23726827 \mathrm{E}+00 & -3.96199949 \mathrm{E}+00 \\ \mathrm{H} & 4.40154232 \mathrm{E}+00 & -1.22568231 \mathrm{E}+00 & 5.83894936 \mathrm{E}-01 \\ \mathrm{H} & 4.80855746 \mathrm{E}+00 & 2.08956039 \mathrm{E}+00 & 6.09813767 \mathrm{E}-01 \\ \mathrm{H} & -2.19854890 \mathrm{E}+00 & -4.70651243 \mathrm{E}+00 & -4.77776913 \mathrm{E}+00 \\ \mathrm{H} & -4.96368731 \mathrm{E}+00 & -6.19965264 \mathrm{E}+00 & -8.28764261 \mathrm{E}-01\end{array}$




$\begin{array}{lrrr}\mathrm{H} & -8.08857430 \mathrm{E}+00 & -2.39337098 \mathrm{E}+00 & 4.64197538 \mathrm{E}-01 \\ \mathrm{H} & -7.19588861 \mathrm{E}+00 & 1.47983343 \mathrm{E}+00 & -2.71884911 \mathrm{E}+00 \\ \mathrm{H} & -3.57091227 \mathrm{E}+00 & 5.35215120 \mathrm{E}-02 & -5.96335728 \mathrm{E}+00 \\ \mathrm{C} & -2.79455669 \mathrm{E}+00 & -1.62324245 \mathrm{E}-01 & 5.78449115 \mathrm{E}+00 \\ \mathrm{H} & -3.01439790 \mathrm{E}+00 & 1.88261437 \mathrm{E}+00 & 5.57233545 \mathrm{E}+00 \\ \mathrm{H} & -4.53586316 \mathrm{E}+00 & -9.23583761 \mathrm{E}-01 & 6.61494757 \mathrm{E}+00 \\ \mathrm{H} & -1.23764834 \mathrm{E}+00 & -5.51408295 \mathrm{E}-01 & 7.09058840 \mathrm{E}+00 \\ \mathrm{H} & 2.77943171 \mathrm{E}+00 & -1.04277747 \mathrm{E}+00 & -3.80572746 \mathrm{E}+00 \\ \mathrm{H} & -5.65665581 \mathrm{E}+00 & 7.22421608 \mathrm{E}+00 & -5.95267142 \mathrm{E}-01 \\ \mathrm{H} & -3.80901238 \mathrm{E}+00 & 6.68895486 \mathrm{E}+00 & 3.55323915 \mathrm{E}+00 \\ \mathrm{H} & -7.30216798 \mathrm{E}+00 & 3.98035781 \mathrm{E}+00 & 2.23357581 \mathrm{E}+00 \\ \mathrm{C} & 7.48803740 \mathrm{E}+00 & 4.87025316 \mathrm{E}-02 & -1.75437239 \mathrm{E}+00 \\ \mathrm{H} & 8.00875045 \mathrm{E}+00 & 1.74813692 \mathrm{E}+00 & -2.87995242 \mathrm{E}+00 \\ \mathrm{H} & 7.41139625 \mathrm{E}+00 & -1.54366984 \mathrm{E}+00 & -3.08490847 \mathrm{E}+00 \\ \mathrm{~N} & 9.43588523 \mathrm{E}+00 & -5.56550405 \mathrm{E}-01 & 1.18199617 \mathrm{E}-01 \\ \mathrm{C} & 9.94933378 \mathrm{E}+00 & 1.55812806 \mathrm{E}+00 & 1.81913255 \mathrm{E}+00 \\ \mathrm{H} & 1.14222281 \mathrm{E}+01 & 1.00525226 \mathrm{E}+00 & 3.16280640 \mathrm{E}+00 \\ \mathrm{H} & 1.05974145 \mathrm{E}+01 & 3.27980569 \mathrm{E}+00 & 8.14068526 \mathrm{E}-01 \\ \mathrm{H} & 8.25984218 \mathrm{E}+00 & 2.03360222 \mathrm{E}+00 & 2.90916327 \mathrm{E}+00 \\ \mathrm{C} & 1.17614332 \mathrm{E}+01 & -1.37239736 \mathrm{E}+00 & -1.13015805 \mathrm{E}+00 \\ \mathrm{H} & 1.13733007 \mathrm{E}+01 & -3.02757070 \mathrm{E}+00 & -2.31062412 \mathrm{E}+00 \\ \mathrm{H} & 1.26179944 \mathrm{E}+01 & 1.08799658 \mathrm{E}-01 & -2.33930845 \mathrm{E}+00 \\ \mathrm{H} & 1.31543938 \mathrm{E}+01 & -1.91758847 \mathrm{E}+00 & 2.99862694 \mathrm{E}-01 \\ \mathrm{C} & -5.41510825 \mathrm{E}-01 & -3.27793672 \mathrm{E}+00 & 3.16251041 \mathrm{E}+00 \\ \mathrm{O} & -4.59261426 \mathrm{E}-01 & -3.85136362 \mathrm{E}+00 & 6.63870499 \mathrm{E}-01 \\ \mathrm{O} & 6.86732937 \mathrm{E}-01 & -4.28052115 \mathrm{E}+00 & 4.81563543 \mathrm{E}+00\end{array}$

TS II-III $: \mathrm{E}=-980.523874153$

Input orientation:

\begin{tabular}{|c|c|c|c|c|c|}
\hline \multirow{2}{*}{$\begin{array}{l}\text { Center } \\
\text { Number }\end{array}$} & \multirow{2}{*}{$\begin{array}{l}\text { Atomic } \\
\text { Number }\end{array}$} & \multirow{2}{*}{$\begin{array}{c}\text { Atomic } \\
\text { Type }\end{array}$} & \multicolumn{3}{|c|}{ Coordinates (Angstroms) } \\
\hline & & & $\mathrm{X}$ & $\mathrm{Y}$ & Z \\
\hline . & 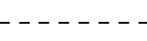 & & -------- & -------- & ------- \\
\hline 1 & 22 & 0 & -.523353 & .065289 & -1.124788 \\
\hline 2 & 7 & 0 & .640989 & -1.046809 & .252752 \\
\hline 3 & 7 & 0 & 1.623222 & .129517 & -1.326109 \\
\hline 4 & 7 & 0 & -.492852 & 1.989582 & -2.231073 \\
\hline 5 & 6 & 0 & 1.790830 & -.581421 & -.185520 \\
\hline 6 & 6 & 0 & 3.127587 & -.780122 & .479788 \\
\hline 7 & 6 & 0 & .431797 & -1.724199 & 1.521105 \\
\hline 8 & 6 & 0 & .195383 & -.752964 & 2.689466 \\
\hline 9 & 14 & 0 & 2.835999 & .958906 & -2.259114 \\
\hline 10 & 6 & 0 & -2.623003 & -.568984 & -2.023513 \\
\hline 11 & 6 & 0 & -2.201382 & -1.607002 & -1.162718 \\
\hline 12 & 6 & 0 & -1.079708 & -2.243317 & -1.760069 \\
\hline 13 & 6 & 0 & -.803782 & -1.598070 & -2.983163 \\
\hline 14 & 6 & 0 & -1.750123 & -.554975 & -3.139739 \\
\hline 15 & 1 & 0 & 3.846277 & -1.200837 & -.230727 \\
\hline 16 & 1 & 0 & 3.067062 & -1.442510 & 1.344508 \\
\hline 17 & 1 & 0 & 3.526681 & .183672 & .816665 \\
\hline 18 & 1 & 0 & 1.275751 & -2.393201 & 1.750054 \\
\hline 19 & 1 & 0 & -.668824 & -. 119514 & 2.461612 \\
\hline 20 & 1 & 0 & 1.065911 & -.089578 & 2.772518 \\
\hline 21 & 1 & 0 & -2.644273 & -1.854524 & -.205665 \\
\hline 22 & 1 & 0 & -3.437096 & .119829 & -1.837591 \\
\hline 23 & 1 & 0 & -1.804085 & .134931 & -3.972698 \\
\hline 24 & 1 & 0 & .012527 & -1.828109 & -3.656561 \\
\hline 25 & 1 & 0 & -.508722 & -3.055337 & -1.329001 \\
\hline 26 & 6 & 0 & -.167447 & 2.546922 & -3.561019 \\
\hline 27 & 1 & 0 & .682347 & 3.233528 & -3.501659 \\
\hline 28 & 1 & 0 & .101567 & 1.717715 & -4.214511 \\
\hline
\end{tabular}




\begin{tabular}{|c|c|c|c|c|c|}
\hline 29 & 1 & 0 & -1.026387 & 3.074409 & -3.988703 \\
\hline 30 & 1 & 0 & -.450358 & -2.367388 & 1.404962 \\
\hline 31 & 1 & 0 & 4.194059 & .327910 & -2.126469 \\
\hline 32 & 1 & 0 & 2.981879 & 2.410131 & -1.885959 \\
\hline 33 & 1 & 0 & 2.451504 & .908745 & -3.706907 \\
\hline 34 & 6 & 0 & -.046970 & -1.502367 & 4.003189 \\
\hline 35 & 1 & 0 & .881305 & -2.034465 & 4.309554 \\
\hline 36 & 1 & 0 & -.809020 & -2.276138 & 3.831446 \\
\hline 37 & 7 & 0 & -.549458 & -.644208 & 5.080981 \\
\hline 38 & 6 & 0 & .430707 & .361270 & 5.488363 \\
\hline 39 & 1 & 0 & .008158 & .969276 & 6.295089 \\
\hline 40 & 1 & 0 & 1.380424 & -.081739 & 5.851851 \\
\hline 41 & 1 & 0 & .657513 & 1.029551 & 4.653567 \\
\hline 42 & 6 & 0 & -.955851 & -1.451696 & 6.229532 \\
\hline 43 & 1 & 0 & -1.729334 & -2.164338 & 5.923103 \\
\hline 44 & 1 & 0 & -.119944 & -2.022421 & 6.682193 \\
\hline 45 & 1 & 0 & -1.379849 & -.800891 & 7.001739 \\
\hline 46 & 6 & 0 & -.971762 & 2.744522 & -1.347737 \\
\hline 47 & 8 & 0 & -1.182426 & 1.092206 & .006935 \\
\hline 48 & 8 & 0 & -1.387107 & 3.662554 & -.756076 \\
\hline
\end{tabular}

Complex III: $E=-980.525438277$

Input orientation:

\begin{tabular}{|c|c|c|c|c|c|}
\hline \multirow{2}{*}{$\begin{array}{l}\text { Center } \\
\text { Number }\end{array}$} & \multirow{2}{*}{$\begin{array}{l}\text { Atomic } \\
\text { Number }\end{array}$} & \multirow{2}{*}{$\begin{array}{c}\text { Atomic } \\
\text { Type }\end{array}$} & \multicolumn{3}{|c|}{ Coordinates (Angstroms) } \\
\hline & & & $\mathrm{X}$ & $\mathrm{Y}$ & Z \\
\hline-- & ---- & & ---- & -------- & -------- \\
\hline 1 & 22 & 0 & 0.499460 & -0.338211 & -1.052043 \\
\hline 2 & 7 & 0 & 0.493425 & 1.075599 & 0.530432 \\
\hline 3 & 7 & 0 & -0.723573 & 1.419008 & -1.280277 \\
\hline 4 & 7 & 0 & -0.898127 & -1.126209 & -2.836685 \\
\hline 5 & 6 & 0 & -0.465067 & 1.800935 & -0.005162 \\
\hline 6 & 6 & 0 & -1.217251 & 2.906722 & 0.689205 \\
\hline 7 & 6 & 0 & 0.865076 & 1.100783 & 1.936006 \\
\hline 8 & 6 & 0 & -0.035320 & 0.217340 & 2.815539 \\
\hline 9 & 14 & 0 & -1.944633 & 2.053412 & -2.345633 \\
\hline 10 & 6 & 0 & 2.137460 & -1.499155 & -2.349552 \\
\hline 11 & 6 & 0 & 2.617218 & -1.433401 & -1.022822 \\
\hline 12 & 6 & 0 & 2.899039 & -0.077567 & -0.737157 \\
\hline 13 & 6 & 0 & 2.644545 & 0.691139 & -1.904254 \\
\hline 14 & 6 & 0 & 2.173097 & -0.183411 & -2.897286 \\
\hline 15 & 1 & 0 & -1.178741 & 3.822366 & 0.090500 \\
\hline 16 & 1 & 0 & -0.818415 & 3.125363 & 1.680755 \\
\hline 17 & 1 & 0 & -2.272677 & 2.631456 & 0.800231 \\
\hline 18 & 1 & 0 & 0.873085 & 2.133443 & 2.317577 \\
\hline 19 & 1 & 0 & -0.019448 & -0.807709 & 2.429865 \\
\hline 20 & 1 & 0 & -1.069216 & 0.575721 & 2.729278 \\
\hline 21 & 1 & 0 & 2.684081 & -2.261468 & -0.329300 \\
\hline 22 & 1 & 0 & 1.817164 & -2.400290 & -2.858406 \\
\hline 23 & 1 & 0 & 1.872569 & 0.101252 & -3.898377 \\
\hline 24 & 1 & 0 & 2.737034 & 1.766769 & -1.989958 \\
\hline 25 & 1 & 0 & 3.256587 & 0.309901 & 0.208080 \\
\hline 26 & 6 & 0 & -0.752021 & -1.039566 & -4.304207 \\
\hline 27 & 1 & 0 & -0.556834 & -0.000777 & -4.568586 \\
\hline 28 & 1 & 0 & 0.094710 & -1.654176 & -4.620878 \\
\hline 29 & 1 & 0 & -1.654555 & -1.373281 & -4.823104 \\
\hline 30 & 1 & 0 & 1.898128 & 0.738060 & 2.006988 \\
\hline 31 & 1 & 0 & -2.188822 & 3.522038 & -2.133460 \\
\hline 32 & 1 & 0 & -3.275338 & 1.361462 & -2.214378 \\
\hline
\end{tabular}




\begin{tabular}{|c|c|c|c|c|c|}
\hline 33 & 1 & 0 & -1.490439 & 1.879710 & -3.763245 \\
\hline 34 & 6 & 0 & 0.425286 & 0.226905 & 4.276216 \\
\hline 35 & 1 & 0 & 0.289408 & 1.243891 & 4.707643 \\
\hline 36 & 1 & 0 & 1.503999 & 0.016454 & 4.305304 \\
\hline 37 & 7 & 0 & -0.228796 & -0.793814 & 5.101648 \\
\hline 38 & 6 & 0 & -1.662510 & -0.549239 & 5.249868 \\
\hline 39 & 1 & 0 & -2.099106 & -1.336088 & 5.873917 \\
\hline 40 & 1 & 0 & -1.888933 & 0.429188 & 5.720953 \\
\hline 41 & 1 & 0 & -2.156758 & -0.586916 & 4.275569 \\
\hline 42 & 6 & 0 & 0.410032 & -0.876730 & 6.413579 \\
\hline 43 & 1 & 0 & 1.472299 & -1.114681 & 6.291128 \\
\hline 44 & 1 & 0 & 0.331267 & 0.061045 & 6.999852 \\
\hline 45 & 1 & 0 & -0.053332 & -1.680701 & 6.995521 \\
\hline 46 & 6 & 0 & -1.741895 & -1.851479 & -2.298441 \\
\hline 47 & 8 & 0 & -0.212517 & -1.540548 & -0.207001 \\
\hline 48 & 8 & 0 & -2.557183 & -2.513316 & -1.796085 \\
\hline
\end{tabular}

TS III-IV $: E=-980.522608186$

Input orientation:

\begin{tabular}{|c|c|c|c|c|c|}
\hline \multirow{2}{*}{$\begin{array}{l}\text { Center } \\
\text { Number }\end{array}$} & \multirow{2}{*}{$\begin{array}{l}\text { Atomic } \\
\text { Number }\end{array}$} & \multirow{2}{*}{$\begin{array}{c}\text { Atomic } \\
\text { Type }\end{array}$} & \multicolumn{3}{|c|}{ Coordinates (Angstroms) } \\
\hline & & & $\mathrm{X}$ & $\mathrm{Y}$ & Z \\
\hline & & & ------- & ------- & ------- \\
\hline 1 & 22 & 0 & -0.717697 & -0.109130 & -0.884656 \\
\hline 2 & 7 & 0 & 0.035487 & -1.234365 & 0.694775 \\
\hline 3 & 7 & 0 & 1.158775 & -0.991639 & -1.214011 \\
\hline 4 & 7 & 0 & 0.816650 & 1.781401 & -3.164822 \\
\hline 5 & 6 & 0 & 1.186783 & -1.426453 & 0.066760 \\
\hline 6 & 6 & 0 & 2.409432 & -2.024728 & 0.714198 \\
\hline 7 & 6 & 0 & -0.156033 & -1.396371 & 2.127572 \\
\hline 8 & 6 & 0 & -0.035316 & -0.068147 & 2.892532 \\
\hline 9 & 14 & 0 & 2.356280 & -1.165637 & -2.474611 \\
\hline 10 & 6 & 0 & -2.643408 & 0.220213 & -2.207193 \\
\hline 11 & 6 & 0 & -3.062901 & -0.374755 & -0.994547 \\
\hline 12 & 6 & 0 & -2.578458 & -1.711639 & -0.972247 \\
\hline 13 & 6 & 0 & -1.867353 & -1.943743 & -2.169514 \\
\hline 14 & 6 & 0 & -1.887079 & -0.744834 & -2.922856 \\
\hline 15 & 1 & 0 & 3.146530 & -2.311114 & -0.038388 \\
\hline 16 & 1 & 0 & 2.158453 & -2.912367 & 1.302690 \\
\hline 17 & 1 & 0 & 2.876142 & -1.296586 & 1.388099 \\
\hline 18 & 1 & 0 & 0.557166 & -2.127911 & 2.534196 \\
\hline 19 & 1 & 0 & -0.780434 & 0.640839 & 2.514517 \\
\hline 20 & 1 & 0 & 0.948756 & 0.368705 & 2.682133 \\
\hline 21 & 1 & 0 & -3.632798 & 0.114464 & -0.213662 \\
\hline 22 & 1 & 0 & -2.830958 & 1.243314 & -2.507186 \\
\hline 23 & 1 & 0 & -1.403653 & -0.591518 & -3.880559 \\
\hline 24 & 1 & 0 & -1.341859 & -2.852986 & -2.433715 \\
\hline 25 & 1 & 0 & -2.713260 & -2.421254 & -0.165186 \\
\hline 26 & 6 & 0 & 0.404590 & 1.957943 & -4.546962 \\
\hline 27 & 1 & 0 & 0.501765 & 1.002628 & -5.067891 \\
\hline 28 & 1 & 0 & -0.644352 & 2.268293 & -4.590032 \\
\hline 29 & 1 & 0 & 1.014005 & 2.702194 & -5.068756 \\
\hline 30 & 1 & 0 & -1.159845 & -1.812744 & 2.284614 \\
\hline 31 & 1 & 0 & 3.034441 & -2.507665 & -2.399119 \\
\hline 32 & 1 & 0 & 3.433922 & -0.121556 & -2.425781 \\
\hline 33 & 1 & 0 & 1.655284 & -1.080196 & -3.793067 \\
\hline 34 & 6 & 0 & -0.240108 & -0.267758 & 4.397358 \\
\hline 35 & 1 & 0 & 0.613224 & -0.844519 & 4.818891 \\
\hline 36 & 1 & 0 & -1.139879 & -0.880212 & 4.553989 \\
\hline 37 & 7 & 0 & -0.448901 & 0.987585 & 5.125387 \\
\hline
\end{tabular}




\begin{tabular}{|c|c|c|c|c|c|}
\hline 38 & 6 & 0 & 0.730765 & 1.851231 & 5.085684 \\
\hline 39 & 1 & 0 & 0.523006 & 2.768731 & 5.645948 \\
\hline 40 & 1 & 0 & 1.630536 & 1.374981 & 5.525752 \\
\hline 41 & 1 & 0 & 0.958120 & 2.136513 & 4.055317 \\
\hline 42 & 6 & 0 & -0.835320 & 0.717297 & 6.508839 \\
\hline 43 & 1 & 0 & -1.750252 & 0.114855 & 6.524802 \\
\hline 44 & 1 & 0 & -0.056202 & 0.177580 & 7.083960 \\
\hline 45 & 1 & 0 & -1.041729 & 1.662819 & 7.021626 \\
\hline 46 & 6 & 0 & 1.378201 & 2.514449 & -2.374783 \\
\hline 47 & 8 & 0 & -0.630598 & 1.411186 & -0.325392 \\
\hline 48 & 8 & 0 & 1.941652 & 3.124737 & -1.545178 \\
\hline
\end{tabular}

\section{Results on chelated complexes}

Chelated complex I with $\mathrm{CH}_{2} \mathrm{CH}_{2} \mathrm{NMe}_{2}: \mathrm{E}=-752.730101922$

Input orientation:

\begin{tabular}{|c|c|c|c|c|c|}
\hline \multirow{2}{*}{$\begin{array}{l}\text { Center } \\
\text { Number }\end{array}$} & \multirow{2}{*}{$\begin{array}{l}\text { Atomic } \\
\text { Number }\end{array}$} & \multirow{2}{*}{$\begin{array}{l}\text { Atomic } \\
\text { Type }\end{array}$} & \multicolumn{3}{|c|}{ Coordinates (Angstroms) } \\
\hline & & & $\mathrm{X}$ & $\mathrm{Y}$ & Z \\
\hline- & 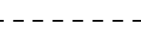 & & --1 & ------- & $-\ldots-\ldots$ \\
\hline 1 & 22 & 0 & .109958 & -.501323 & .007815 \\
\hline 2 & 14 & 0 & -3.269435 & -.051225 & 1.315849 \\
\hline 3 & 7 & 0 & .409778 & -.733108 & 1.661325 \\
\hline 4 & 7 & 0 & -1.941979 & .333583 & .257707 \\
\hline 5 & 7 & 0 & -.293307 & 1.465228 & -.640128 \\
\hline 6 & 7 & 0 & 2.204466 & .787616 & -.244481 \\
\hline 7 & 6 & 0 & .610506 & -1.246050 & 2.983727 \\
\hline 8 & 6 & 0 & -1.494883 & 1.544786 & -.086704 \\
\hline 9 & 6 & 0 & .607666 & 2.546766 & -.949421 \\
\hline 10 & 6 & 0 & 1.944933 & 1.857756 & -1.244933 \\
\hline 11 & 6 & 0 & 2.454578 & 1.396750 & 1.083131 \\
\hline 12 & 6 & 0 & 3.412913 & .033971 & -.635461 \\
\hline 13 & 1 & 0 & .297600 & 3.123244 & -1.835372 \\
\hline 14 & 1 & 0 & .713775 & 3.267868 & -.122867 \\
\hline 15 & 1 & 0 & 2.776694 & 2.581917 & -1.266251 \\
\hline 16 & 1 & 0 & 1.885858 & 1.374060 & -2.226128 \\
\hline 17 & 1 & 0 & 2.707069 & .607190 & 1.791084 \\
\hline 18 & 1 & 0 & 3.281019 & 2.125627 & 1.027898 \\
\hline 19 & 1 & 0 & 1.551135 & 1.884757 & 1.448152 \\
\hline 20 & 1 & 0 & 3.579193 & -.771131 & .084209 \\
\hline 21 & 1 & 0 & 4.303000 & .684195 & -.653108 \\
\hline 22 & 1 & 0 & 3.277875 & -.403182 & -1.626847 \\
\hline 23 & 1 & 0 & 1.464180 & -.761194 & 3.488113 \\
\hline 24 & 1 & 0 & -.272277 & -1.074086 & 3.618930 \\
\hline 25 & 1 & 0 & .808261 & -2.331090 & 2.983760 \\
\hline 26 & 1 & 0 & -3.496127 & -1.529538 & 1.259174 \\
\hline 27 & 1 & 0 & -3.027613 & .330936 & 2.752182 \\
\hline 28 & 1 & 0 & -4.548661 & .629968 & .898664 \\
\hline 29 & 6 & 0 & -2.214638 & 2.843755 & .171557 \\
\hline 30 & 1 & 0 & -1.912899 & 3.256580 & 1.143251 \\
\hline 31 & 1 & 0 & -1.979208 & 3.587441 & -.595831 \\
\hline 32 & 1 & 0 & -3.297380 & 2.694747 & .193338 \\
\hline 33 & 6 & 0 & -.047545 & -2.851495 & -.356138 \\
\hline 34 & 6 & 0 & -1.138500 & -2.282489 & -1.048670 \\
\hline 35 & 1 & 0 & -2.182149 & -2.389290 & -.783340 \\
\hline 36 & 6 & 0 & -.632771 & -1.508260 & -2.123993 \\
\hline 37 & 1 & 0 & -1.222904 & -.915486 & -2.811549 \\
\hline 38 & 6 & 0 & 1.141447 & -2.405874 & -.983283 \\
\hline 39 & 1 & 0 & 2.148189 & -2.671802 & -.685957 \\
\hline 40 & 6 & 0 & .773145 & -1.587720 & -2.086724 \\
\hline
\end{tabular}




\begin{tabular}{|c|c|c|c|c|c|}
\hline 41 & 1 & 0 & 1.450470 & -1.099871 & -2.777786 \\
\hline 42 & 1 & 0 & -.105894 & -3.480211 & .522976 \\
\hline
\end{tabular}

TS for $\mathrm{CO}_{2}$ addition from below on Chelated Complex with $\mathrm{CH}_{2} \mathrm{CH}_{2} \mathrm{NMe}_{2}$ :

$E=-941.225000333$

Input orientation:

\begin{tabular}{|c|c|c|c|c|c|}
\hline \multirow{2}{*}{$\begin{array}{l}\text { Center } \\
\text { Number }\end{array}$} & \multirow{2}{*}{$\begin{array}{l}\text { Atomic } \\
\text { Number }\end{array}$} & \multirow{2}{*}{$\begin{array}{l}\text { Atomic } \\
\text { Type }\end{array}$} & \multicolumn{3}{|c|}{ Coordinates (Angstroms) } \\
\hline & & & $\mathrm{X}$ & $\mathrm{Y}$ & $\mathrm{Z}$ \\
\hline-- & -1 & & ---------1 & --------- & --------- \\
\hline 1 & 22 & 0 & -0.525827 & -0.239506 & 0.230808 \\
\hline 2 & 14 & 0 & -0.191364 & -0.111629 & -3.456689 \\
\hline 3 & 7 & 0 & -0.696825 & 1.519543 & 0.204843 \\
\hline 4 & 7 & 0 & 0.217183 & -0.526056 & -1.799146 \\
\hline 5 & 7 & 0 & 1.347938 & -1.085350 & -0.011751 \\
\hline 6 & 7 & 0 & 0.715934 & -0.134667 & 2.379677 \\
\hline 7 & 6 & 0 & -1.584654 & 2.616853 & 0.529187 \\
\hline 8 & 6 & 0 & 1.425105 & -0.826620 & -1.314850 \\
\hline 9 & 6 & 0 & 2.446392 & -1.142897 & 0.925837 \\
\hline 10 & 6 & 0 & 1.774547 & -1.179071 & 2.297917 \\
\hline 11 & 6 & 0 & 1.347761 & 1.210770 & 2.422477 \\
\hline 12 & 6 & 0 & -0.030667 & -0.310875 & 3.644253 \\
\hline 13 & 1 & 0 & 3.061873 & -2.046366 & 0.798811 \\
\hline 14 & 1 & 0 & 3.114851 & -0.274704 & 0.836097 \\
\hline 15 & 1 & 0 & 2.506897 & -1.049296 & 3.111041 \\
\hline 16 & 1 & 0 & 1.284041 & -2.150840 & 2.427606 \\
\hline 17 & 1 & 0 & 0.570080 & 1.957749 & 2.588886 \\
\hline 18 & 1 & 0 & 2.083022 & 1.260874 & 3.242580 \\
\hline 19 & 1 & 0 & 1.820283 & 1.444806 & 1.469172 \\
\hline 20 & 1 & 0 & -0.827532 & 0.434092 & 3.699576 \\
\hline 21 & 1 & 0 & 0.632854 & -0.177639 & 4.513372 \\
\hline 22 & 1 & 0 & -0.472248 & -1.307543 & 3.694029 \\
\hline 23 & 1 & 0 & -1.078401 & 3.369722 & 1.154477 \\
\hline 24 & 1 & 0 & -1.918533 & 3.125287 & -0.383690 \\
\hline 25 & 1 & 0 & -2.468336 & 2.268447 & 1.080403 \\
\hline 26 & 1 & 0 & -1.599243 & 0.384209 & -3.468023 \\
\hline 27 & 1 & 0 & 0.720008 & 0.910712 & -4.065082 \\
\hline 28 & 1 & 0 & -0.117959 & -1.342534 & -4.323904 \\
\hline 29 & 6 & 0 & 2.705783 & -0.795895 & -2.095533 \\
\hline 30 & 1 & 0 & 2.543433 & -1.094138 & -3.135423 \\
\hline 31 & 1 & 0 & 3.094896 & 0.229886 & -2.092036 \\
\hline 32 & 1 & 0 & 3.460132 & -1.454166 & -1.655994 \\
\hline 33 & 6 & 0 & -2.915290 & -0.356388 & 0.147324 \\
\hline 34 & 6 & 0 & -2.434410 & -1.335765 & -0.746791 \\
\hline 35 & 1 & 0 & -2.549713 & -1.318629 & -1.822076 \\
\hline 36 & 6 & 0 & -1.739948 & -2.321535 & -0.000930 \\
\hline 37 & 1 & 0 & -1.216689 & -3.176647 & -0.409728 \\
\hline 38 & 6 & 0 & -2.493991 & -0.714126 & 1.450129 \\
\hline 39 & 1 & 0 & -2.704319 & -0.162688 & 2.357827 \\
\hline 40 & 6 & 0 & -1.779737 & -1.942020 & 1.353986 \\
\hline 41 & 1 & 0 & -1.332752 & -2.494056 & 2.170409 \\
\hline 42 & 1 & 0 & -3.467788 & 0.532522 & -0.126201 \\
\hline 43 & 6 & 0 & 0.641504 & 2.303982 & -1.003259 \\
\hline 44 & 8 & 0 & -0.016810 & 2.869489 & -1.827504 \\
\hline 45 & 8 & 0 & 1.747368 & 2.044276 & -0.600008 \\
\hline
\end{tabular}

Complex I with $\mathrm{CH}_{2} \mathrm{CH}_{2} \mathrm{CH}_{2} \mathrm{NMe}_{2}: \mathrm{E}=-792.019512341$

Z-Matrix orientation: 


\begin{tabular}{|c|c|c|c|c|c|}
\hline \multirow{2}{*}{$\begin{array}{l}\text { Center } \\
\text { Number }\end{array}$} & \multirow{2}{*}{$\begin{array}{l}\text { Atomic } \\
\text { Number }\end{array}$} & \multirow{2}{*}{$\begin{array}{l}\text { Atomic } \\
\text { Type }\end{array}$} & \multicolumn{3}{|c|}{ Coordinates (Angstroms) } \\
\hline & & & $\mathrm{X}$ & $\mathrm{Y}$ & Z \\
\hline---- & 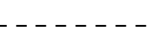 & & ------- & ------- & -------- \\
\hline 1 & 22 & 0 & 0.039007 & -0.567437 & 0.050466 \\
\hline 2 & 14 & 0 & 3.444717 & 0.042006 & -0.796813 \\
\hline 3 & 7 & 0 & 0.263152 & -1.461582 & -1.374767 \\
\hline 4 & 7 & 0 & 1.921746 & 0.520090 & -0.114968 \\
\hline 5 & 7 & 0 & 0.028880 & 1.608859 & 0.122546 \\
\hline 6 & 6 & 0 & -2.849705 & 1.118457 & 0.076426 \\
\hline 7 & 6 & 0 & 0.540701 & -2.419919 & -2.402761 \\
\hline 8 & 6 & 0 & 1.345844 & 1.717602 & 0.038196 \\
\hline 9 & 6 & 0 & -0.848491 & 2.660636 & 0.587558 \\
\hline 10 & 6 & 0 & -2.232574 & 2.514783 & -0.050841 \\
\hline 11 & 1 & 0 & -0.958856 & 2.602419 & 1.685396 \\
\hline 12 & 1 & 0 & -0.452236 & 3.662861 & 0.364335 \\
\hline 13 & 1 & 0 & -2.185775 & 2.809960 & -1.105444 \\
\hline 14 & 1 & 0 & -2.922803 & 3.218752 & 0.434260 \\
\hline 15 & 1 & 0 & -0.240524 & -2.424187 & -3.183092 \\
\hline 16 & 1 & 0 & 1.493070 & -2.202743 & -2.910695 \\
\hline 17 & 1 & 0 & 0.606787 & -3.446990 & -2.006433 \\
\hline 18 & 1 & 0 & 3.824248 & -1.285304 & -0.215318 \\
\hline 19 & 1 & 0 & 3.407738 & -0.098271 & -2.294245 \\
\hline 20 & 1 & 0 & 4.555937 & 1.013044 & -0.482383 \\
\hline 21 & 6 & 0 & 2.099832 & 3.021254 & 0.149298 \\
\hline 22 & 1 & 0 & 1.683467 & 3.780924 & -0.521721 \\
\hline 23 & 1 & 0 & 2.038061 & 3.414593 & 1.171625 \\
\hline 24 & 1 & 0 & 3.154786 & 2.882368 & -0.093481 \\
\hline 25 & 6 & 0 & -0.667862 & -2.363956 & 1.438833 \\
\hline 26 & 6 & 0 & 0.748466 & -2.345023 & 1.445696 \\
\hline 27 & 1 & 0 & 1.397180 & -3.079960 & 0.987019 \\
\hline 28 & 6 & 0 & 1.162127 & -1.175816 & 2.126962 \\
\hline 29 & 1 & 0 & 2.185792 & -0.855380 & 2.265642 \\
\hline 30 & 6 & 0 & -1.120489 & -1.209041 & 2.125617 \\
\hline 31 & 1 & 0 & -2.153797 & -0.943207 & 2.313161 \\
\hline 32 & 6 & 0 & 0.009597 & -0.474241 & 2.550534 \\
\hline 33 & 1 & 0 & -0.003502 & 0.469103 & 3.082523 \\
\hline 34 & 1 & 0 & -1.287789 & -3.130193 & 0.990573 \\
\hline 35 & 1 & 0 & -2.881644 & 0.823008 & 1.131832 \\
\hline 36 & 1 & 0 & -3.893593 & 1.170691 & -0.282047 \\
\hline 37 & 7 & 0 & -2.162773 & 0.017788 & -0.669202 \\
\hline 38 & 6 & 0 & -3.045436 & -1.172063 & -0.629764 \\
\hline 39 & 1 & 0 & -3.267194 & -1.441778 & 0.404782 \\
\hline 40 & 1 & 0 & -3.995505 & -0.973100 & -1.152134 \\
\hline 41 & 1 & 0 & -2.531086 & -2.006240 & -1.110887 \\
\hline 42 & 6 & 0 & -2.001453 & 0.404208 & -2.093560 \\
\hline 43 & 1 & 0 & -2.950970 & 0.790169 & -2.501073 \\
\hline 44 & 1 & 0 & -1.221058 & 1.159625 & -2.187481 \\
\hline 45 & 1 & 0 & -1.685285 & -0.472003 & -2.658015 \\
\hline
\end{tabular}

TS for $\mathrm{CO}_{2}$ addition from below on chelated complex with $\mathrm{CH}_{2} \mathrm{CH}_{2} \mathrm{CH}_{2} \mathrm{NMe}_{2}$ : $\mathrm{E}=-980.508708533$

Input orientation:

\begin{tabular}{|c|c|c|c|c|c|}
\hline \multirow{2}{*}{$\begin{array}{l}\text { Center } \\
\text { Number }\end{array}$} & \multirow{2}{*}{$\begin{array}{l}\text { Atomic } \\
\text { Number }\end{array}$} & \multirow{2}{*}{$\begin{array}{c}\text { Atomic } \\
\text { Type }\end{array}$} & \multicolumn{3}{|c|}{ Coordinates (Angstroms) } \\
\hline & & & $\mathrm{X}$ & $\mathrm{Y}$ & $\mathrm{Z}$ \\
\hline---- & -- & & -------- & -------- & -------- \\
\hline 1 & 22 & 0 & -0.460890 & 0.375596 & -0.023965 \\
\hline 2 & 14 & 0 & 0.107173 & -0.182855 & 3.416677 \\
\hline 3 & 7 & 0 & -1.390027 & -1.116185 & 0.275218 \\
\hline 4 & 7 & 0 & 0.590621 & 0.391083 & 1.840530 \\
\hline
\end{tabular}




\begin{tabular}{|c|c|c|c|c|c|}
\hline 5 & 7 & 0 & 1.643441 & 0.628926 & -0.070327 \\
\hline 6 & 6 & 0 & 1.137942 & 0.444002 & -2.941687 \\
\hline 7 & 6 & 0 & -2.758163 & -1.580200 & 0.392368 \\
\hline 8 & 6 & 0 & 1.781777 & 0.488564 & 1.242367 \\
\hline 9 & 6 & 0 & 2.714064 & 0.986931 & -0.975143 \\
\hline 10 & 6 & 0 & 2.538916 & 0.312820 & -2.339340 \\
\hline 11 & 1 & 0 & 2.721157 & 2.081562 & -1.122557 \\
\hline 12 & 1 & 0 & 3.698867 & 0.717017 & -0.568636 \\
\hline 13 & 1 & 0 & 2.821614 & -0.743072 & -2.271194 \\
\hline 14 & 1 & 0 & 3.242086 & 0.772373 & -3.047229 \\
\hline 15 & 1 & 0 & -2.918239 & -2.477424 & -0.218200 \\
\hline 16 & 1 & 0 & -2.991766 & -1.868164 & 1.427309 \\
\hline 17 & 1 & 0 & -3.478661 & -0.811820 & 0.081495 \\
\hline 18 & 1 & 0 & -1.026798 & 0.685407 & 3.880902 \\
\hline 19 & 1 & 0 & -0.370396 & -1.600140 & 3.418558 \\
\hline 20 & 1 & 0 & 1.215235 & -0.055638 & 4.426800 \\
\hline 21 & 6 & 0 & 3.107611 & 0.443807 & 1.954687 \\
\hline 22 & 1 & 0 & 3.685719 & -0.426921 & 1.624402 \\
\hline 23 & 1 & 0 & 3.700154 & 1.340972 & 1.743202 \\
\hline 24 & 1 & 0 & 2.967247 & 0.371910 & 3.033632 \\
\hline 25 & 6 & 0 & -2.382826 & 1.564814 & -0.729030 \\
\hline 26 & 6 & 0 & -2.356868 & 1.614277 & 0.686183 \\
\hline 27 & 1 & 0 & -3.052833 & 1.128620 & 1.357197 \\
\hline 28 & 6 & 0 & -1.235592 & 2.382972 & 1.072696 \\
\hline 29 & 1 & 0 & -0.919393 & 2.569638 & 2.089045 \\
\hline 30 & 6 & 0 & -1.280232 & 2.317750 & -1.207417 \\
\hline 31 & 1 & 0 & -1.030051 & 2.499841 & -2.244232 \\
\hline 32 & 6 & 0 & -0.567449 & 2.816306 & -0.095448 \\
\hline 33 & 1 & 0 & 0.345058 & 3.397770 & -0.129085 \\
\hline 34 & 1 & 0 & -3.118754 & 1.050651 & -1.333699 \\
\hline 35 & 1 & 0 & 0.829722 & 1.496090 & -2.943121 \\
\hline 36 & 1 & 0 & 1.180736 & 0.112266 & -3.994060 \\
\hline 37 & 7 & 0 & 0.058444 & -0.341097 & -2.262549 \\
\hline 38 & 6 & 0 & -1.148525 & -0.291115 & -3.125682 \\
\hline 39 & 1 & 0 & -1.434204 & 0.741058 & -3.330502 \\
\hline 40 & 1 & 0 & -0.955860 & -0.796678 & -4.084858 \\
\hline 41 & 1 & 0 & -1.969541 & -0.795308 & -2.612678 \\
\hline 42 & 6 & 0 & 0.464789 & -1.770922 & -2.201381 \\
\hline 43 & 1 & 0 & 0.820873 & -2.106702 & -3.188379 \\
\hline 44 & 1 & 0 & 1. 242690 & -1.917422 & -1.454737 \\
\hline 45 & 1 & 0 & -0.398825 & -2.368848 & -1.909829 \\
\hline 46 & 6 & 0 & -0.253100 & -2.707462 & 0.652200 \\
\hline 47 & 8 & 0 & 0.873197 & -2.339267 & 0.856781 \\
\hline 48 & 8 & 0 & -1.005460 & -3.632547 & 0.552201 \\
\hline
\end{tabular}

